\title{
Crystal Engineering of Molecular Solids as Temporary Adhesives
}

Nicholas D. Blelloch, ${ }^{\dagger}$ Haydn T. Mitchell, ${ }^{\dagger}$ Carly C. Tymm, ${ }^{\dagger}$ Douglas W. Van Citters, ${ }^{\ddagger}$ and Katherine A. Mirica*, ${ }^{+}$

${ }^{+}$Burke Laboratory, Department of Chemistry, Dartmouth College, Hanover, New Hampshire 03755, United States

* Thayer School of Engineering, Dartmouth College, Hanover, New Hampshire 03755, United States

(106 pages)

\begin{tabular}{|c|c|c|}
\hline & Table of Contents & S1 \\
\hline SI. & General Materials & S2 \\
\hline SII. & Physical Properties Molecular Solids & S3 \\
\hline SIII. & Cleaning Glass Substrates & S15 \\
\hline SIV. & Melt-Bonding Substrates with Molecular Solids & S15 \\
\hline SV. & $\begin{array}{l}\text { Cross Polarized Light Microscopy of Polycrystalline Films of } \\
\text { Molecular Solids }\end{array}$ & S20 \\
\hline SVI. & $\begin{array}{l}\text { Obtaining and Analyzing Optical Light Micrographs of Overlap } \\
\text { Joints Before and After Shearing the Assembly }\end{array}$ & S30 \\
\hline SVII. & Shear Testing of the Overlap Joint for Adhesive Measurements & S39 \\
\hline SVIII. & Quantifying the Shear Moduli of Adhesives in Overlap Joints & S40 \\
\hline SIX. & Micrographs Obtained by White Light Interferometry & S40 \\
\hline SX. & Powder X-Ray Diffraction & S49 \\
\hline SXI. & $\begin{array}{l}\text { Quantifying the Mechanism of Failure for Melt-Bonded } \\
\text { Samples }\end{array}$ & S63 \\
\hline SXII. & Generating Electron Surface Potential Plots & S64 \\
\hline SXIII. & Generating Hirshfeld Surfaces and Fingerprint Plots & S64 \\
\hline
\end{tabular}




\begin{tabular}{|c|l|c|}
\hline SXIV. & Casting Pellets of Molecular Solids for Cohesive Measurements & S64 \\
\hline SXV. & Shear Testing of Pellets for Cohesive Measurements & S65 \\
\hline SXVI. & $\begin{array}{l}\text { Melt-Bonding Substrates with Molecular Solids for Release of } \\
\text { Adherends by Sublimation }\end{array}$ \\
\hline SXVII. & Release of Adherends by Sublimation & S73 \\
\hline SXVIII. & Thermal Analysis of Molecular Solids & S74 \\
\hline SXIX. & Modification of Substrate Surface Chemistry \\
\hline SXX. & Characterization of Glass Adherends & S90 \\
\hline SXXI. & Optical Analysis of Bulk-Shear Samples & S92 \\
\hline SXXII. & $\begin{array}{l}\text { Single Crystal Growth and Structure Determination of (-)- } \\
\text { Menthol }\end{array}$ & S94 \\
\hline SXXIII. & $\begin{array}{l}\text { Samples } \\
\text { SXIV. }\end{array}$ & References \\
\hline
\end{tabular}

\section{SI. General Materials}

The molecular solids used as temporary adhesives were iodine (BDH Chemicals, 99.8\%, Product \#:BDH4620, CAS \#: 7553-56-2; Crystallography Open Database ID: 1010091), octacyclic sulfur (Alfa Aesar, 99.5\%, Product \#: AA33394-36, CAS \#: 770434-9; Cambridge Structural Database (CSD) Reference: FURHUV), naphthalene (TCI America, 98\%, Product \#: TCN0885, CAS \#: 91-20-3; CSD Reference: NAPHTA15), hexachlorobenzene (Sigma-Aldrich, analytical standard, Product \#: 45522, CAS \#: 118-74-1; CSD Reference: HCLBNZ12), (+)-camphor (Spectrum Chemicals, 98\%, 
Product \#: 70003, CAS \#: 464-49-3; CSD Reference: UGAHUF), dimethyl sulfone (Sigma-Aldrich, 98\%, Product \#: M81705, CAS \#: 67-71-0; CSD Reference: DMSLLO04), (-)-menthol (TCI America, 99\%, Product \#: TCM0545, CAS \#: 2216-515), and (S)-ibuprofen (Enzo Life Sciences, 98.5\%, Product \#: 98157-946, CAS \#: 51146-56-6; CSD Reference: IBPRAC05). All molecular solids and solvents used were purchased from commercial vendors and used as received. Glass slides (Gold Seal Products, ThermoFisher Scientific, Cat. \# 3051) were used as substrates and melt-bonded samples were handled and stored using aluminum foil (Reynolds Wrap) to minimize contamination of surfaces and sublimation of the molecular solids. The thickness and mass of each glass slide was measured using a digital display micrometer (iGaging) and balance (VWR 124B), respectively. A chemicallyresistant polytetrafluoroethylene washer (McMaster-Carr, Product No. 95630A410) was used to set the thickness of the adhesive layer during meltbonding. Melt-bonding was performed on a lab hot plate (IKA RCT Basic) thermocoupled to a custom bonding frame.

\section{SII. Physical Properties of Molecular Solids}

Table S1. Molar mass, melting point, boiling point, enthalpy of fusion and enthalpy of sublimation for molecular solids. The molar mass $\left(\mathrm{MM}, \mathrm{g} \mathrm{mol}^{-1}\right)$, melting point $\left(\mathrm{MP},{ }^{\circ} \mathrm{C}\right)$, boiling point $\left(B P,{ }^{\circ} \mathrm{C}\right)$, enthalpy of fusion $\left(\Delta \mathrm{H}_{\text {fus }}, \mathrm{kJ} \mathrm{mol}^{-1}\right)$ and enthalpy of sublimation $\left(\Delta \mathrm{H}_{\text {sub }}, \mathrm{kJ}\right.$ $\mathrm{mol}^{-1}$ ) were used to develop structure-property relationships and determine whether the molecular solid met the selection criteria. The MM was calculated from the molecular structure. The MP and BP were retrieved from the digital archive called Chemistry Webbook organized and maintained by National Institute of Standards and Technology. ${ }^{\mathrm{S1}}$ 


\begin{tabular}{|c|c|c|c|c|c|c|c|}
\hline Molecular solid & $\begin{array}{c}\mathrm{MM} \\
\left(\mathrm{g} \mathrm{mol}^{-1}\right)\end{array}$ & $\begin{array}{l}\mathrm{MP} \\
\left({ }^{\circ} \mathrm{C}\right)\end{array}$ & $\begin{array}{l}\mathrm{BP} \\
\left({ }^{\circ} \mathrm{C}\right)\end{array}$ & $\begin{array}{c}\Delta \mathrm{H}_{\text {fus }} \\
\left(\mathrm{kJ} \mathrm{mol}{ }^{-1}\right)\end{array}$ & 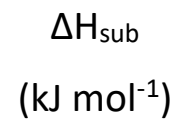 & $\begin{array}{l}\text { Ref \# } \\
\Delta \mathrm{H}_{\text {fus }}\end{array}$ & $\begin{array}{l}\text { Ref \# } \\
\Delta \mathrm{H}_{\text {sub }}\end{array}$ \\
\hline lodine & 127 & 113 & 184 & 16 & 55 & S2 & S3 \\
\hline Octacyclic sulphur & 257 & 117 & 445 & 17 & 100 & S4 & S4 \\
\hline Naphthalene & 128 & 80 & 218 & 19 & 71 & S5 & S6 \\
\hline Hexachlorobenzene & 285 & 231 & 322 & 74 & 97 & S7 & S8 \\
\hline \multicolumn{8}{|c|}{$\begin{array}{l}\text { Table S1. Molar mass, melting point, boiling point, enthalpy of fusion and enthalpy of } \\
\text { sublimation for molecular solids (continued). }\end{array}$} \\
\hline Molecular solid & $\mathrm{MM}$ & MP & BP & $\Delta \mathrm{H}_{\text {fus }}$ & $\Delta \mathrm{H}_{\text {sub }}$ & Ref \# & Ref \# \\
\hline & $\left(\mathrm{g} \mathrm{mol}^{-1}\right)$ & $\left({ }^{\circ} \mathrm{C}\right)$ & $\left({ }^{\circ} \mathrm{C}\right)$ & $\left(\mathrm{kJ} \mathrm{mol}^{-1}\right)$ & $\left(\mathrm{kJ} \mathrm{mol}^{-1}\right)$ & $\Delta \mathrm{H}_{\text {fus }}$ & $\Delta \mathrm{H}_{\text {sub }}$ \\
\hline Camphor & 152 & 175 & 209 & 7 & 52 & S9 & S10 \\
\hline Dimethyl sulfone & 94 & 109 & 238 & 18 & 77 & S11 & S12 \\
\hline (-)-Menthol & 186 & 40 & 212 & 10 & 90 & s13 & S13 \\
\hline Ibuprofen & 256 & 76 & 157 & 28 & 116 & S14 & S15 \\
\hline
\end{tabular}

Kinetics of sublimation are important to the rapid release of substrates by sublimable adhesives. The kinetics of sublimation are a function of vapor pressure. ${ }^{516}$ The equilibrium vapor pressure of a solid at a given temperature can be estimated using the Clausius-Clapeyron equation (Eq. S1), $\Delta \mathrm{H}_{\text {sub }}$, and another equilibrium vapor pressure for a defined temperature. ${ }^{\mathrm{S16}}$

$$
\ln \left(\frac{P_{1}}{P_{2}}\right)=\frac{\Delta H_{\text {vap }}}{R}\left(\frac{1}{T_{2}}-\frac{1}{T_{1}}\right)
$$

The vapor pressure of each molecular solid selected was estimated and tabulated in Table S2. Crystal structures, and relevant structural information for each molecular solid are presented in Figures S9-S9. ${ }^{17-23}$ 
Table S2. Vapor pressure of selected molecular solids. The vapor pressure $\left(\mathrm{VP}_{2}\right.$, $\mathrm{Pa}$ ) was estimated for each selected molecular solid at $298.15 \mathrm{~K}\left(25^{\circ} \mathrm{C}\right)$ using the Clausius-Clapeyron equation (Eq. S1), ${ }^{\mathrm{S} 16}$ enthalpy of sublimation $\left(\Delta \mathrm{H}_{\mathrm{sub}}, \mathrm{kJ} \mathrm{mol}^{-1}\right)$ from Table S1 and a vapor pressure ( $\left.\mathrm{VP}_{1}, \mathrm{~Pa}\right)$ of the solid at a defined temperature $\left(T_{1}, K\right)$ obtained from the literature.

\begin{tabular}{ccccc}
\hline Molecular solid & $\mathrm{VP}_{2}(\mathrm{~Pa}, 298 \mathrm{~K})$ & $\mathrm{T}_{1}(\mathrm{~K})$ & $\mathrm{VP}_{1}(\mathrm{~Pa})$ & Ref for $\mathrm{T}_{1}$ and $\mathrm{VP}_{1}$ \\
\hline lodine & 804 & 338 & 807 & $\mathrm{~S}^{24}$ \\
Octacyclic sulphur & $1.0 \times 10^{-7}$ & 312 & $1.0 \times 10^{-5}$ & $\mathrm{~S} 4$ \\
Table S2. Vapor pressure of selected molecular solids (continued). & \\
\hline Molecular solid & $\mathrm{VP}_{2}(\mathrm{~Pa}, 298 \mathrm{~K})$ & $\mathrm{T}_{1}(\mathrm{~K})$ & $\mathrm{VP}_{1}(\mathrm{~Pa})$ & Ref for $\mathrm{T}_{1}$ and VP \\
\hline Naphthalene & 2.7 & 290 & 2.7 & $\mathrm{~S}^{25}$ \\
Hexachlorobenzene & 1.4 & 358 & 1.4 & $\mathrm{~S}^{25}$ \\
Camphor & 87 & 298 & 87 & $\mathrm{~S}^{26}$ \\
\hline Dimethyl sulfone & 990 & 440 & 10,000 & $\mathrm{~S}^{26}$ \\
(-) -Menthol & 0.001 & 298 & 0.001 & $\mathrm{~S}^{27}$ \\
Ibuprofen & $6.3 \times 10^{-6}$ & 298 & $6.3 \times 10^{-6}$ & $\mathrm{~S}^{28}$ \\
\hline
\end{tabular}




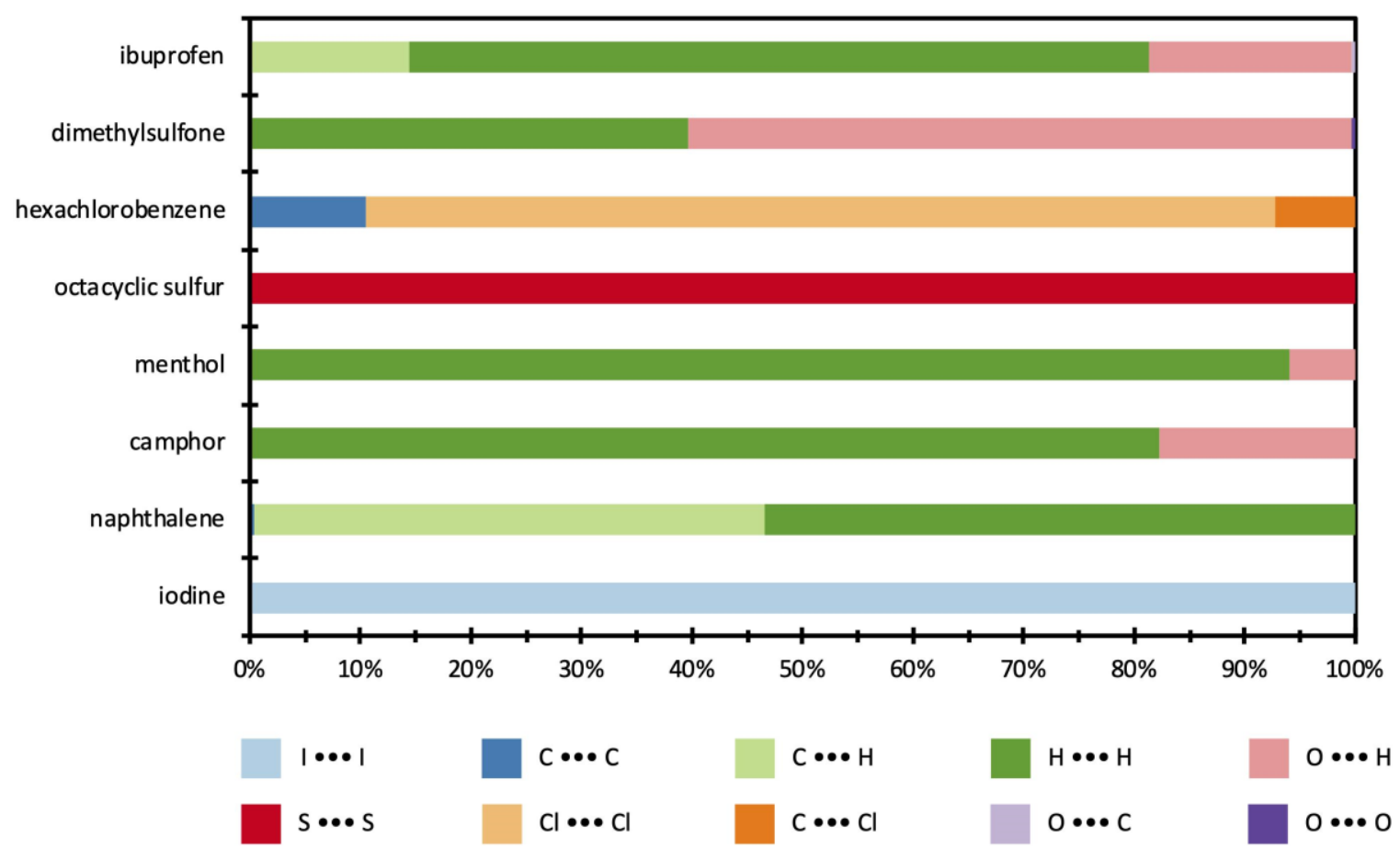

Figure S1. Quantification of the intermolecular interactions present within each molecular solid. Percentage of each interaction were calculated using Hirshfeld Surface Analysis in CrystalExplorer (see SI Section SXII for more detail). 
A)

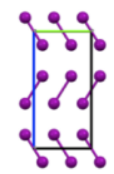

B)

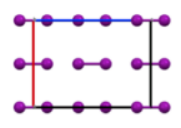

C)

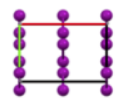

D)

\begin{tabular}{|c|c|c|c|c|c|c|}
\hline $\begin{array}{c}\text { space } \\
\text { group }\end{array}$ & $\mathrm{a}(\AA \AA)$ & $\mathrm{b}(\AA))$ & $\mathrm{c}(\AA)$ & $\alpha\left(^{\circ}\right)$ & $\beta\left(^{\circ}\right)$ & $\gamma\left({ }^{\circ}\right)$ \\
\hline Cmca (64) & 7.255 & 4.795 & 9.78 & 90 & 90 & 90 \\
\hline
\end{tabular}

Figure S2. Unit cell of iodine. A) Unit cell as viewed down the crystallographic a-axis.

B) Unit cell as viewed down the crystallographic b-axis. C) Unit cell as viewed down the crystallographic c-axis. D) Structural data for the unit cell. 
A)

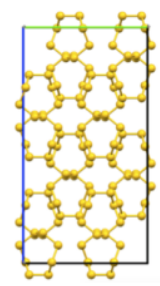

B)

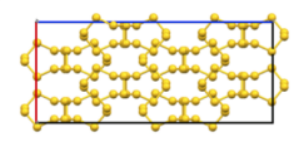

C)

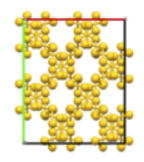

D)

\begin{tabular}{|c|c|c|c|c|c|c|}
\hline $\begin{array}{c}\text { space } \\
\text { group }\end{array}$ & $\mathrm{a}(\mathrm{A})$ & $\mathrm{b}(\AA)$ & $\mathrm{c}(\AA \AA)$ & $\alpha\left(^{\circ}\right)$ & $\beta\left(^{\circ}\right)$ & $\gamma\left({ }^{\circ}\right)$ \\
\hline Fddd (70) & $10.4646(1)$ & $12.8660(1)$ & $24.4860(3)$ & 90 & 90 & 90 \\
\hline
\end{tabular}

Figure S3. Unit cell of octacyclic sulfur. A) Unit cell as viewed down the crystallographic a-axis. B) Unit cell as viewed down the crystallographic b-axis. C) Unit cell as viewed down the crystallographic c-axis. D) Structural data for the unit cell. 
A)

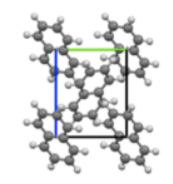

B)

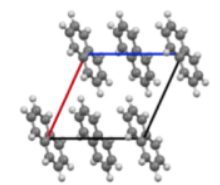

C)

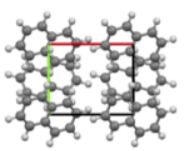

\section{D)}

\begin{tabular}{|c|c|c|c|c|c|c|}
\hline $\begin{array}{c}\text { space } \\
\text { group }\end{array}$ & $\mathrm{a}(\AA \AA)$ & $\mathrm{b}(\AA \AA)$ & $\mathrm{c}(\AA ̊)$ & $\alpha\left(^{\circ}\right)$ & $\beta\left(^{\circ}\right)$ & $\gamma\left({ }^{\circ}\right)$ \\
\hline $\mathrm{P} 2_{1} / \mathrm{c}(14)$ & $7.8249(2)$ & $5.9349(1)$ & $8.0997(2)$ & 90 & $114.441(2)$ & 90 \\
\hline
\end{tabular}

Figure S4. Unit cell of naphthalene. A) Unit cell as viewed down the crystallographic aaxis. B) Unit cell as viewed down the crystallographic b-axis. C) Unit cell as viewed down the crystallographic c-axis. D) Structural data for the unit cell. 
A)

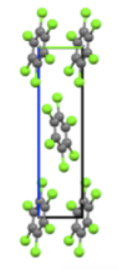

B)

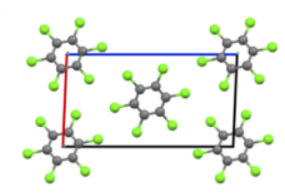

C)

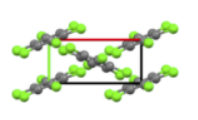

D)

\begin{tabular}{|c|c|c|c|c|c|c|}
\hline $\begin{array}{c}\text { space } \\
\text { group }\end{array}$ & $\mathrm{a}(\AA \AA)$ & $\mathrm{b}(\AA \AA)$ & $\mathrm{c}(\AA \AA)$ & $\alpha\left(^{\circ}\right)$ & $\beta\left(^{\circ}\right)$ & $\gamma\left(^{\circ}\right)$ \\
\hline $\mathrm{P} 2_{1} / \mathrm{n}(14)$ & $7.967(3)$ & $3.7609(14)$ & $14.670(5)$ & 90 & $92.459(6)$ & 90 \\
\hline
\end{tabular}

Figure S5. Unit cell of hexachlorobenzene. A) Unit cell as viewed down the crystallographic a-axis. B) Unit cell as viewed down the crystallographic b-axis. C) Unit cell as viewed down the crystallographic c-axis. D) Structural data for the unit cell. 
A)

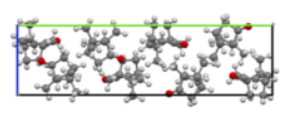

B)

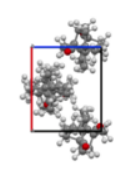

C)

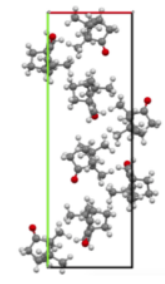

D)

\begin{tabular}{|c|c|c|c|c|c|c|}
\hline $\begin{array}{c}\text { space } \\
\text { group }\end{array}$ & $\mathrm{a}(\AA)$ & $\mathrm{b}(\AA \AA)$ & $\mathrm{c}(\AA)$ & $\alpha\left(^{\circ}\right)$ & $\beta\left(^{\circ}\right)$ & $\gamma\left({ }^{\circ}\right)$ \\
\hline $\begin{array}{c}\mathrm{P} 2_{1} 2_{1} 2_{1} \\
(19)\end{array}$ & $8.9277(2)$ & $27.0359(5)$ & $7.3814(1)$ & 90 & 90 & 90 \\
\hline
\end{tabular}

Figure S6. Unit cell of (+)-camphor. A) Unit cell as viewed down the crystallographic aaxis. B) Unit cell as viewed down the crystallographic b-axis. C) Unit cell as viewed down the crystallographic c-axis. D) Structural data for the unit cell. 
A)

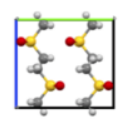

B)

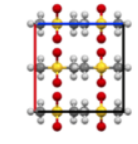

C)

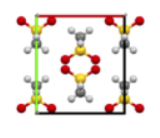

D)

\begin{tabular}{|c|c|c|c|c|c|c|}
\hline $\begin{array}{c}\text { space } \\
\text { group }\end{array}$ & $a(\AA)$ & $b(\AA)$ & $c(\AA)$ & $\alpha\left(^{\circ}\right)$ & $\beta\left(^{\circ}\right)$ & $\gamma\left(^{\circ}\right)$ \\
\hline Cmcm (63) & $7.186(2)$ & $7.965(3)$ & $7.215(3)$ & 90 & 90 & 90 \\
\hline
\end{tabular}

Figure S7. Unit cell of dimethyl sulfone. A) Unit cell as viewed down the crystallographic a-axis. B) Unit cell as viewed down the crystallographic b-axis. C) Unit cell as viewed down the crystallographic c-axis. D) Structural data for the unit cell. 
A)

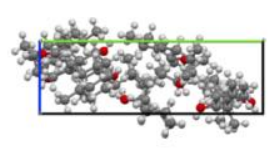

B)

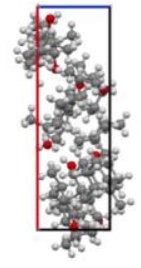

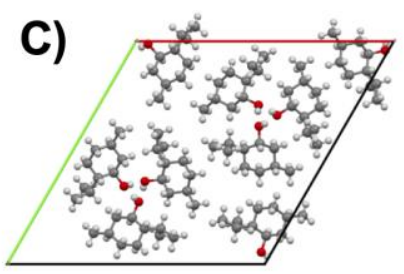

D)

\begin{tabular}{|c|c|c|c|c|c|c|}
\hline $\begin{array}{c}\text { space } \\
\text { group }\end{array}$ & $a(\AA)$ & $b(\AA)$ & $c(\AA)$ & $\alpha\left(^{\circ}\right)$ & $\beta\left(^{\circ}\right)$ & $\gamma\left(^{\circ}\right)$ \\
\hline $\mathrm{P3}_{1}(144)$ & $21.452(4)$ & $21.452(4)$ & $6.0355(10)$ & 90 & 90 & 120 \\
\hline
\end{tabular}

Figure S8. Unit cell of (-)-menthol. A) Unit cell as viewed down the crystallographic aaxis. B) Unit cell as viewed down the crystallographic b-axis. C) Unit cell as viewed down the crystallographic c-axis. D) Structural data for the unit cell. 
A)

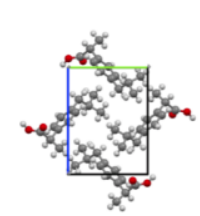

B)

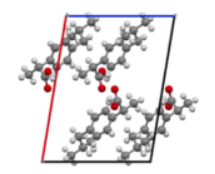

C)

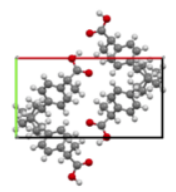

D)

\begin{tabular}{|c|c|c|c|c|c|c|}
\hline $\begin{array}{c}\text { space } \\
\text { group }\end{array}$ & $\mathrm{a}(\AA \AA)$ & $\mathrm{b}(\AA \AA)$ & $\mathrm{c}(\AA)$ & $\alpha\left(^{\circ}\right)$ & $\beta\left(^{\circ}\right)$ & $\gamma\left(\left(^{\circ}\right)\right.$ \\
\hline $\mathrm{P2}_{1} / \mathrm{c}(14)$ & 14.667 & 7.886 & 10.730 & 90 & 99.362 & 90 \\
\hline
\end{tabular}

Figure S9. Unit cell of (S)-ibuprofen. A) Unit cell as viewed down the crystallographic a-axis. B) Unit cell as viewed down the crystallographic b-axis. C) Unit cell as viewed down the crystallographic c-axis. D) Structural data for the unit cell. 


\section{SIII. Cleaning Glass Substrates}

To remove contaminants (e.g., lint, dust, or adsorbed molecules from the laboratory environment) from the surface of the glass slides, glass slides were washed with water, ethanol, and acetone, in that order, and then treated using a plasma cleaner (Harrick Plasma, Model PDC-32G). The selection and order of the solvent ensured removal of contaminants with a range of solubility, no drying residue or "coffee rings" and minimal drying time. Once washed the glass slides were laid out on aluminum foil in a fume hood. Once dried, plasma cleaner treatment entailed exposing the glass slide to the "HI" RF (radiofrequency electromagnetic radiation) setting for one minute. The glass slides where then stored in plastic petri dishes immediately after treatment for 24 hours to avoid contamination and allow the reactive species on the surface of the glass to equilibrate with air before being melt-bonded.

\section{SIV. Melt-Bonding Substrates with Molecular Solids}

Substrates were melt-bonded using an aluminum fixture (Aluminum Alloy 6061, Fig. S10) to hold the substrates in place while the molecular solid was melted by heating with a hotplate (IKA RCT Basic). The aluminum fixture was designed in E-Z Cam and fabricated in a Trak DPM2 Bed Mill. The aluminum fixture was designed to be thermocoupled to the hotplate for greater control and accuracy of the temperature during melt-bonding. Lastly, PTFE washers (thickness $=0.002-0.004 "$ ) were placed between the glass substrates during melt-bonding while the glass slides were pressed 
down to expel excess melted molecular solid and force the adhesive layer to be the thickness of the PTFE washer for each assembly. 


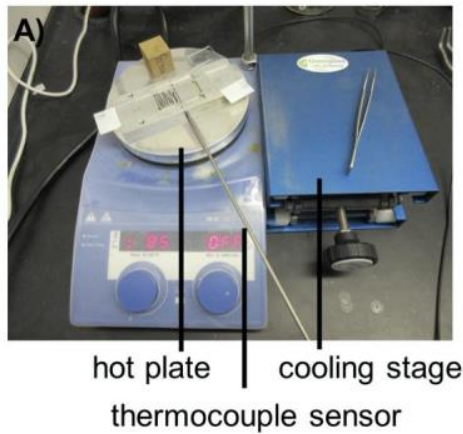

Figure S10. Procedure for controlling thickness of polycrystalline film during meltbonding of two glass substrates using a molecular solid. (a) Glass substrates were preheated to 10 to $15{ }^{\circ} \mathrm{C}$ above the melting point of the molecular solid on an aluminium fixture that is thermocoupled to a hotplate. (b) A powder of molecular solid was deposited on the overlap area marked by permanent marker. A PTFE washer (c) was placed at the edge of the overlap area. The top substrate was then placed on top of the molecular solid and washer. Once the molecular solid melts a mass was placed on top of the top substrate to expel excess molecular solid and to confine the melt to the thickness of the PTFE washer. The aluminium fixture and assembly were then moved to the cooling stage to allow the molecular solid to crystallize between the glass substrates. After the assembly has cooled the PTFE washer was removed. (d) Detailed photograph of the PTFE washer. 
The aluminum fixture and substrates were preheated at the melt-bonding temperature before placing the molecular solid between the glass slides. The bottom substrate was placed in the lower step while the top substrate was placed perpendicular to the upper step, resting on the small lip around the upper step during preheat. Once the hotplate signaled it had reached the set temperature the molecular solid was placed on the bottom substrate in the designated overlap area $\left(20 \times 25 \mathrm{~mm}^{2}\right)$ demarked using permanent marker on the aluminum fixture. The mass of molecular solid deposited on the bottom substrate was held constant and summarized in Table S3. A clean PTFE washer was then placed on the bottom substrate at the edge of the overlap area. The top substrate was then placed over the molecular solid and designated overlap area. A mass of $112.3 \mathrm{~g}$ was placed on top of the top substrate to flatten the assembly, spread the melted molecular solid, and pushed the top substrate down onto the PTFE washer to set the thickness of adhesive layer. The fixture is then transferred from the hot plate to a lab jack (ChemGlass, CG-3054). The lab jack acted as a cooling stage for the fixture and assembly of substrates and melted molecular solid. As the melted molecular solid cooled it fused and bonded the substrates. The PTFE washer was removed after the molecular solid was cooled which left a small defect in the adhesive layer. Ten assemblies were produced for each molecular solid, but some assemblies were not included in the final results for any values due to fracture due to typical handling or being excluded by a Dixon's Q-test with a 90\% confidence interval on either the stress or strain. All micrographs of the disregarded assemblies were labelled "failed Q-test". 
Table S3. Summary of mass and thickness of polycrystalline films of molecular solids between glass substrates. The average mass and thickness of the polycrystalline film were controlled using PTFE washers to physically limit the mass and thickness of the film. Ten overlap joints were melt-bonded for each molecular solid. The number of bonded assemblies that made it through mechanical testing without the glass fracturing are noted.

\begin{tabular}{cccc}
\hline Molecular solid & $\mathrm{n}$ & mass $(\mathrm{mg})$ & film thickness $(\mu \mathrm{m})$ \\
\hline lodine & 10 & $180 \pm 39$ & $58 \pm 9$ \\
Octacyclic sulphur & 9 & $36 \pm 7$ & $52 \pm 7$ \\
Naphthalene & 10 & $29 \pm 7$ & $60 \pm 6$ \\
Hexachlorobenzene & 7 & $27 \pm 10$ & $70 \pm 10$ \\
Camphor & 7 & $20 \pm 3$ & $51 \pm 6$ \\
Dimethyl sulfone & 10 & $35 \pm 6$ & $72 \pm 10$ \\
(-)-Menthol & 7 & $32 \pm 6$ & $61 \pm 9$ \\
Ibuprofen & 8 & $29 \pm 5$ & $84 \pm 10$ \\
\hline
\end{tabular}




\section{SV. Cross Polarized Light Microscopy of Polycrystalline Films of Molecular Solids}

Cross Polarized Light (XPL) micrographs were obtained using an XPL microscope (Nikon E200 POL) for three assemblies from each set of ten assemblies produced for each adhesive. Each assembly was placed on the rotatable stage of the XPL microscope with the bottom substrate rested on the stage and the unbonded end of the bottom substrate pointed towards the microscope. For each assembly selected a series of XPL micrographs were obtained at $0^{\circ}, 45^{\circ}$, and $90^{\circ}$ in a region with a defining feature of interest (e.g., a boundary between crystallites or voids). Additionally, XPL micrographs were obtained at the "top", "middle", and "bottom" of the adhesive layer as labelled in Figure S11. A standard scale bar was imaged to assist in determining the scale of features in samples

(Fig. S12). The XPL micrographs obtained at $0^{\circ}, 45^{\circ}$, and $90^{\circ}$ are organized in Figures S13S20. 


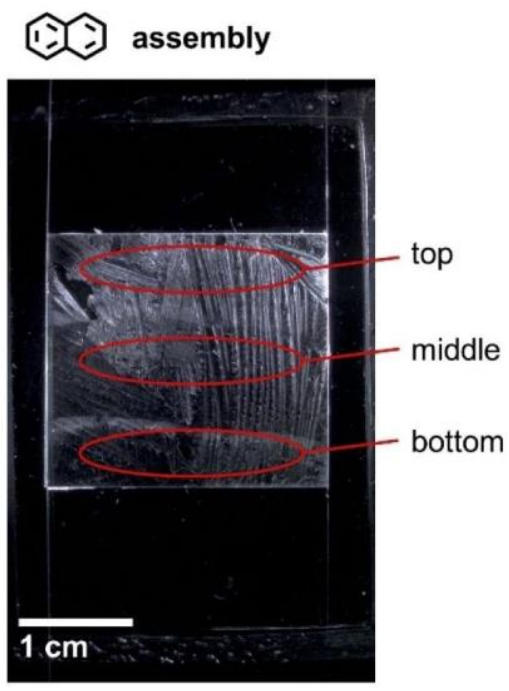

Figure S11. Optical Light micrograph of polycrystalline film of naphthalene between two glass substrates with the "top", "middle", and "bottom" labeled. The "top", "middle", and "bottom" are the regions where XPL micrographs were captured to characterize the variation in crystal morphology and organization within and between assemblies.

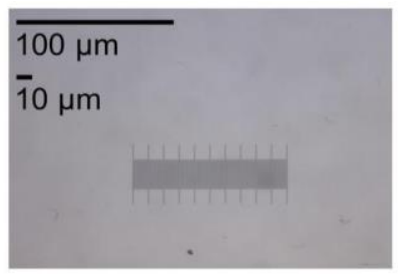

Figure S12. Cross Polarized Light micrograph of the standard scale bar used to assist in determining the scale in each micrograph obtained. The small markings, large markings, and total length of the scale bar are $1 \mu \mathrm{m}, 10 \mu \mathrm{m}$, and $100 \mu \mathrm{m}$, respectively. 
A)
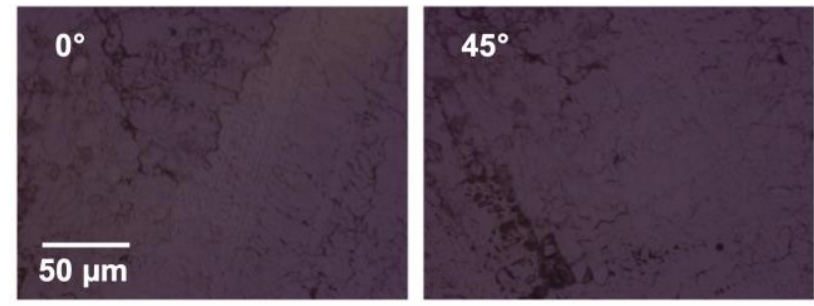

top

B)

\section{$\overline{50 \mu m}$}

C)

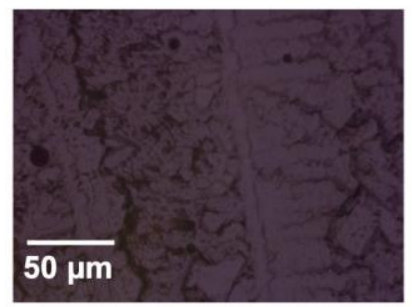

D)

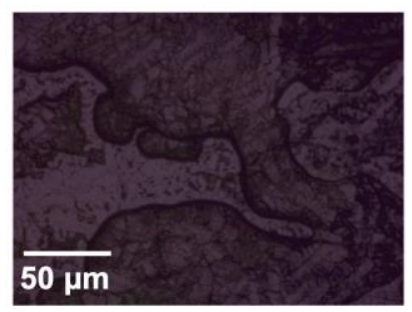

$90^{\circ}$

middle
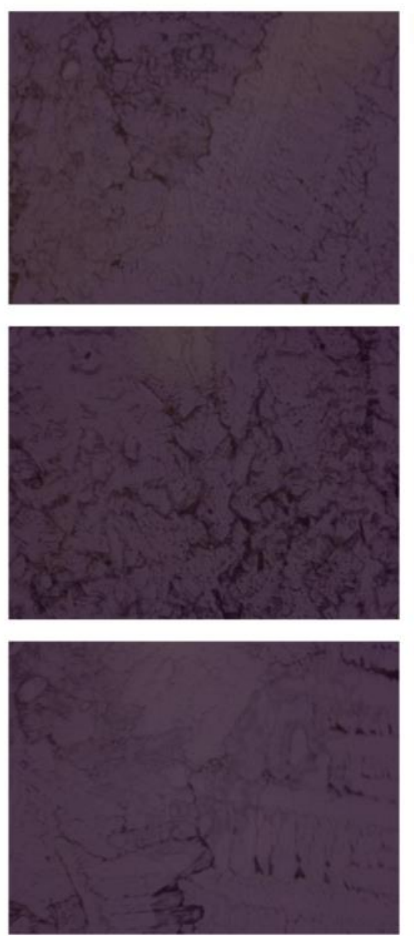

bottom
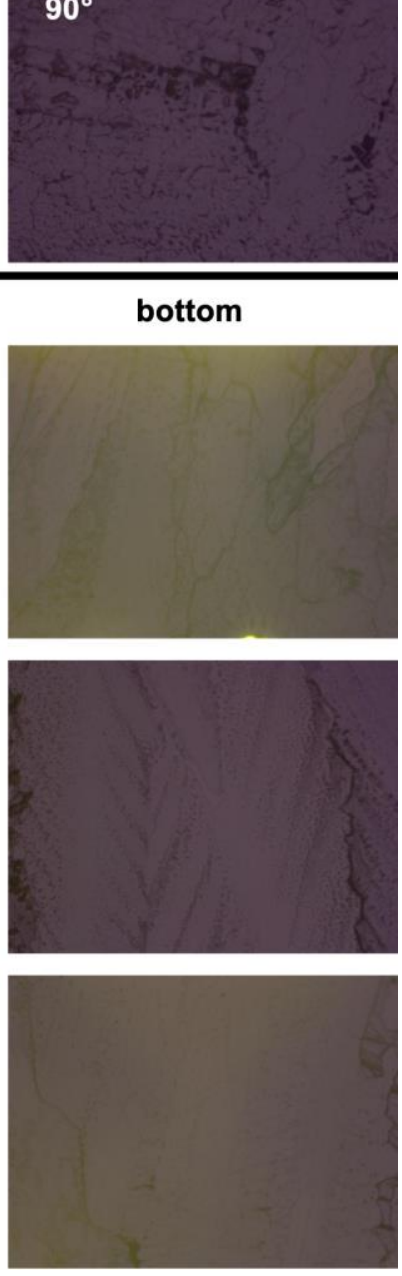

Figure S13. Polycrystalline films of iodine grown from the melt between two glass

substrates exhibit crystals with an acicular crystal habit. (a) XPL micrographs were obtained of the same area of the polycrystalline film at $0^{\circ}, 45^{\circ}$, and $90^{\circ}$ to qualitatively demonstrate the crystallites are in different orientations. (b), (c), and (d) are XPL micrographs of three areas near the top, middle, and bottom of a sample along the center of the sample for three samples. 
A)
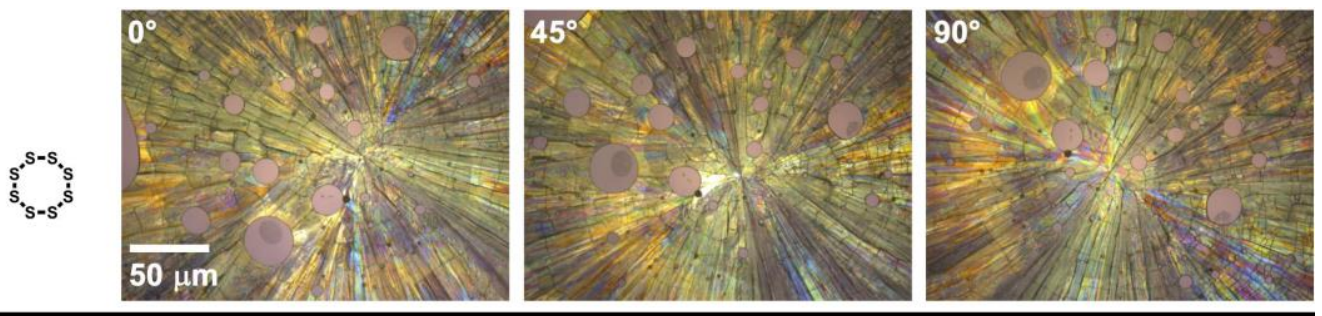

top

middle
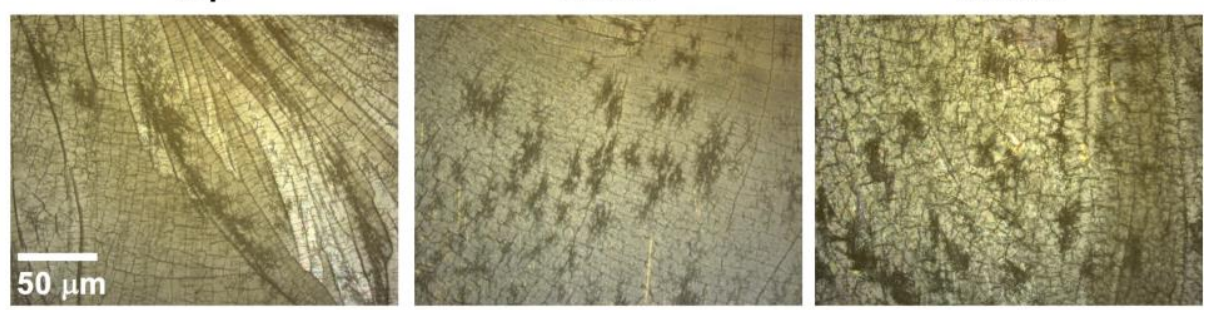

C)
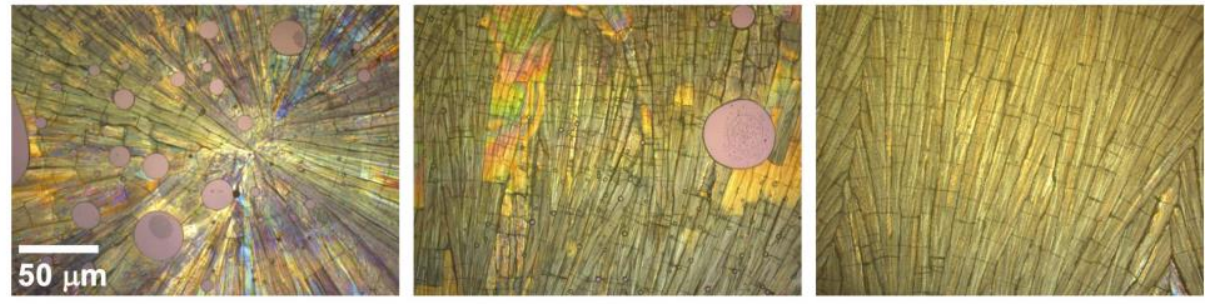

D)
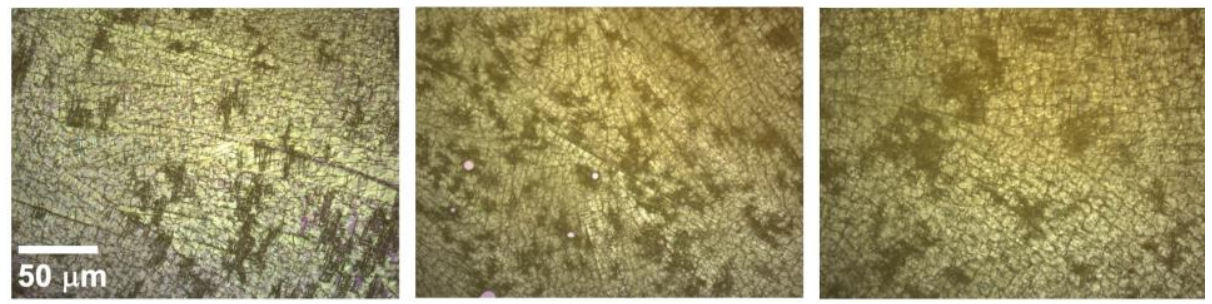

Figure S14. Polycrystalline films of octacyclic sulfur grown from the melt between

two glass substrates exhibit crystals with an acicular crystal habit. (a) XPL micrographs were obtained of the same area of the polycrystalline film at $0^{\circ}, 45^{\circ}$, and $90^{\circ}$ to qualitatively demonstrate the crystallites are in different orientations. (b), (c), and (d) are XPL micrographs of three areas near the top, middle, and bottom of a sample along the center of the sample for three samples. 
A)
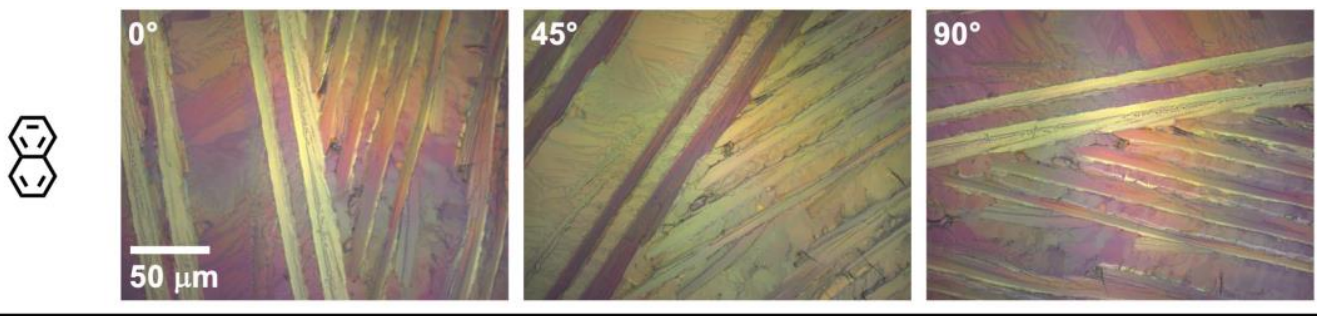

top

middle

bottom

B)
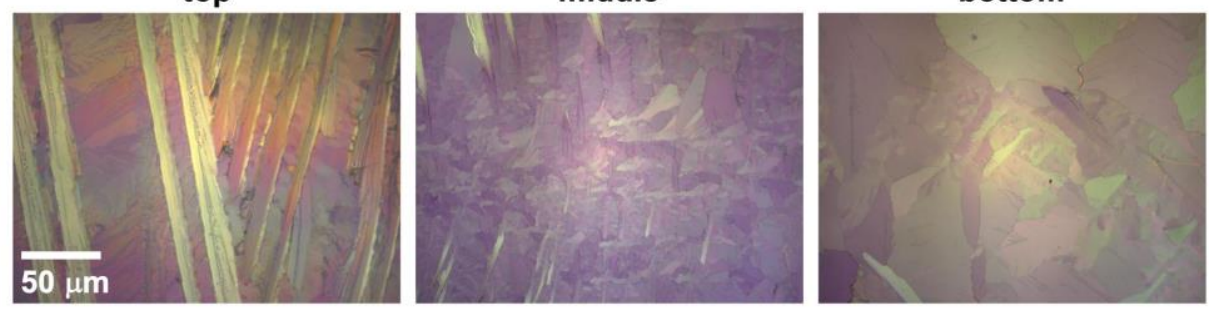

C)
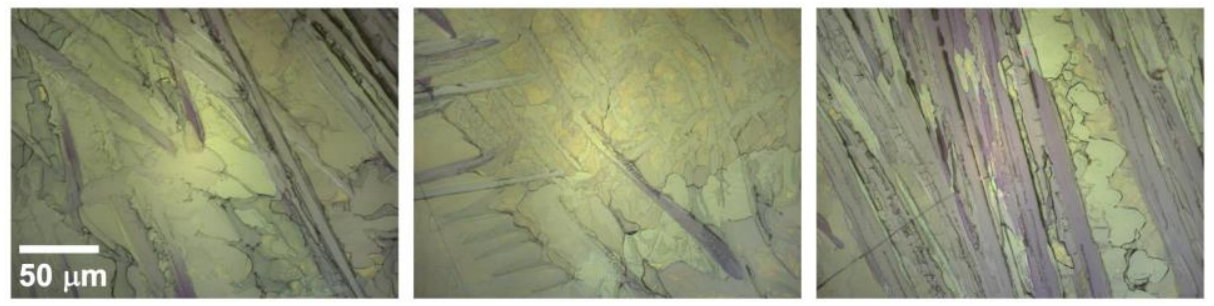

D)
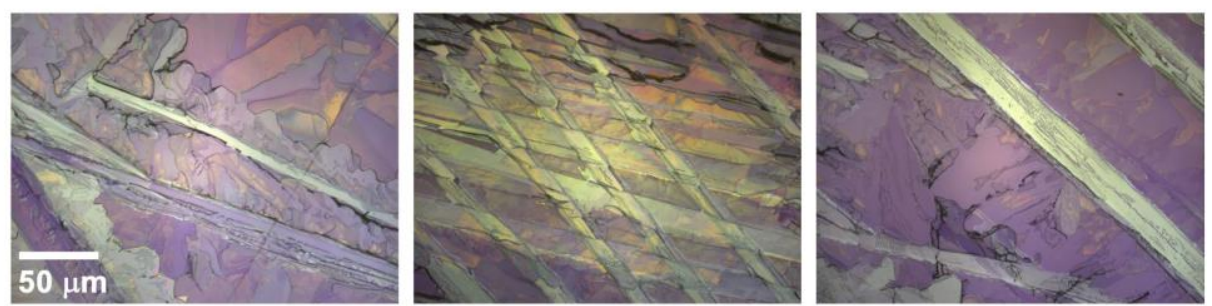

Figure S15. Polycrystalline films of naphthalene grown from the melt between two glass substrates exhibit crystals with an acicular crystal habit. (a) XPL micrographs were obtained of the same area of the polycrystalline film at $0^{\circ}, 45^{\circ}$, and $90^{\circ}$ to qualitatively demonstrate the crystallites are in different orientations. (b), (c), and (d) are XPL micrographs of three areas near the top, middle, and bottom of a sample along the center of the sample for three samples. 
A)
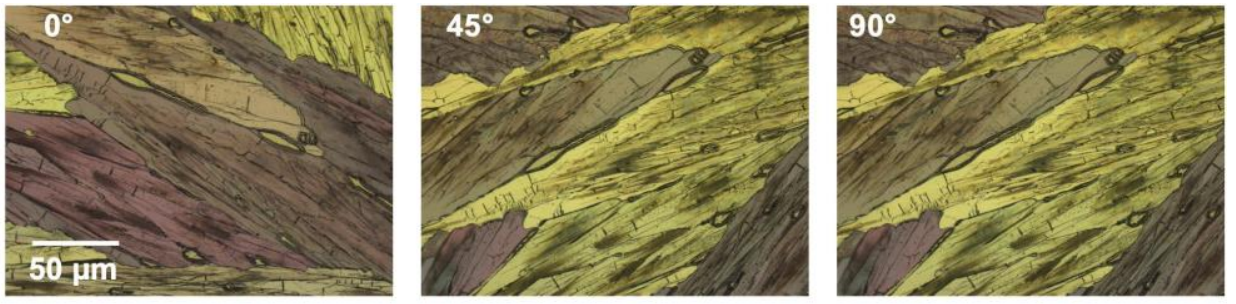

top

middle

bottom

B)
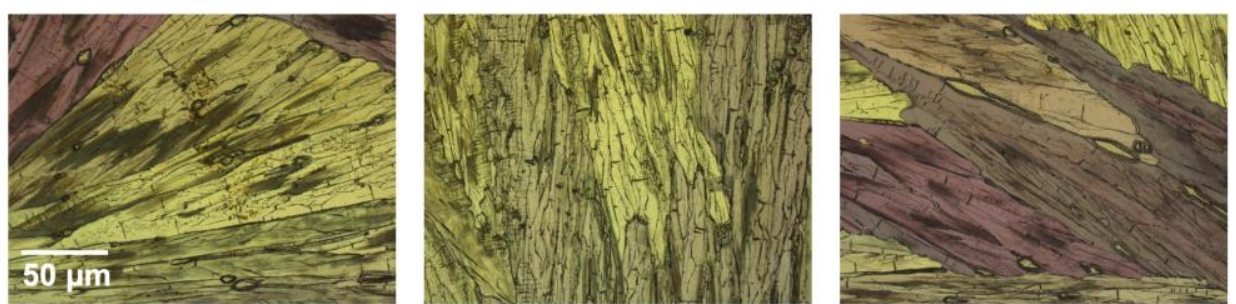

C)
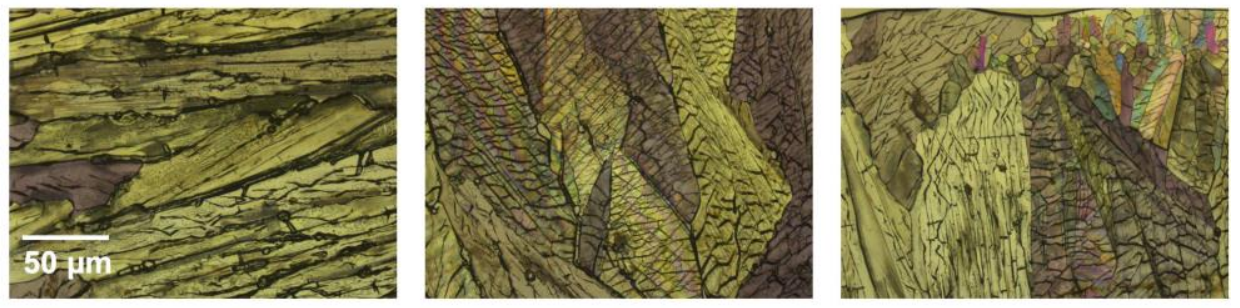

D)
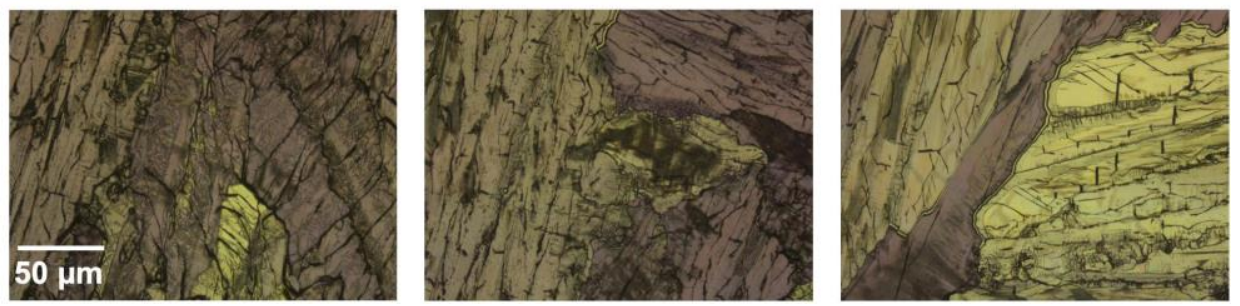

Figure S16. Polycrystalline films of hexachlorobenzene grown from the melt between two glass substrates exhibit crystals with an acicular crystal habit. (a) XPL micrographs were obtained of the same area of the polycrystalline film at $0^{\circ}, 45^{\circ}$, and $90^{\circ}$ to qualitatively demonstrate the crystallites are in different orientations. (b), (c), and (d) are XPL micrographs of three areas near the top, middle, and bottom of a sample along the center of the sample for three samples. 
A)
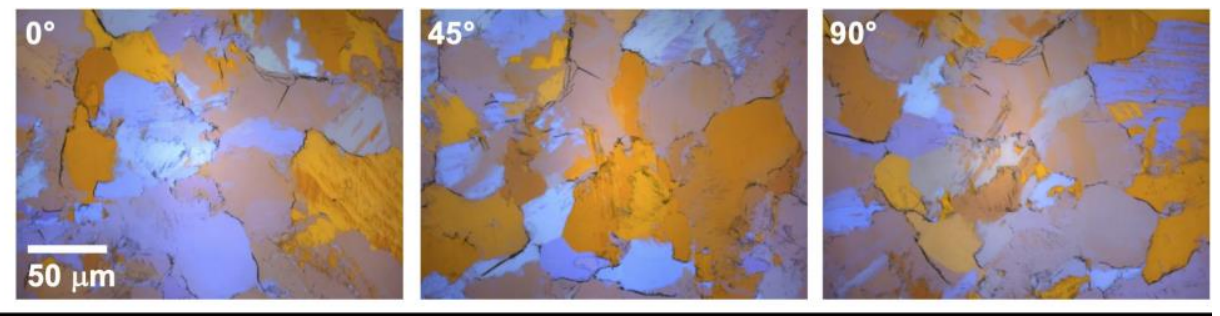

B)
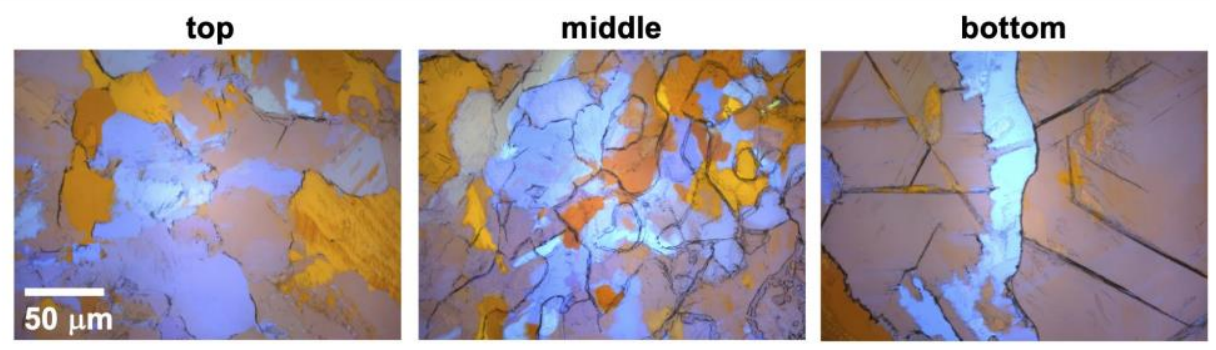

C)
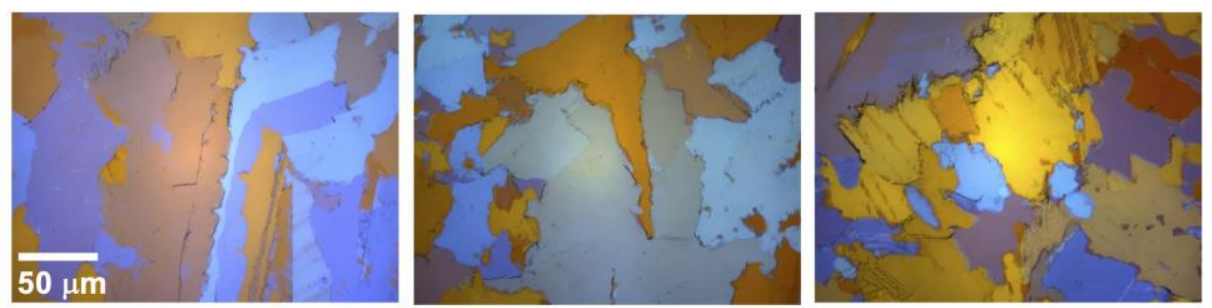

D)
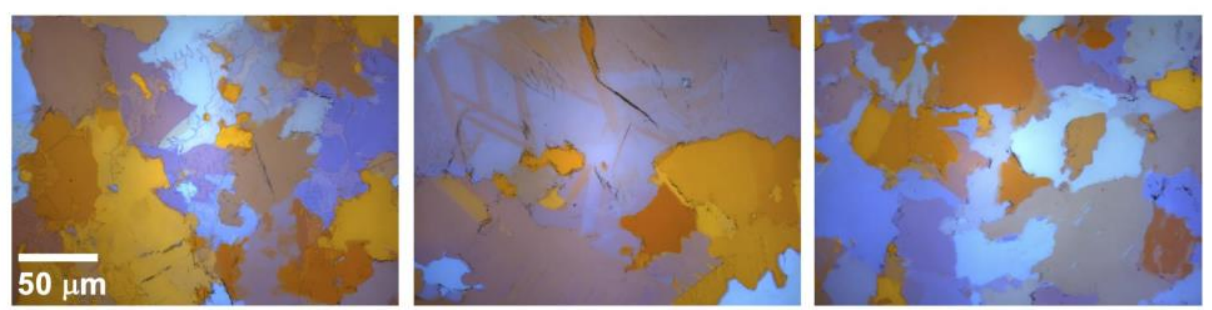

Figure S17. Polycrystalline films of $(+)$-camphor grown from the melt between two glass substrates exhibit crystals with a globular crystal habit. (a) XPL micrographs were obtained of the same area of the polycrystalline film at $0^{\circ}, 45^{\circ}$, and $90^{\circ}$ to qualitatively demonstrate the crystallites are in different orientations. (b), (c), and (d) are XPL micrographs of three areas near the top, middle, and bottom of a sample along the center of the sample for three samples. 
A)
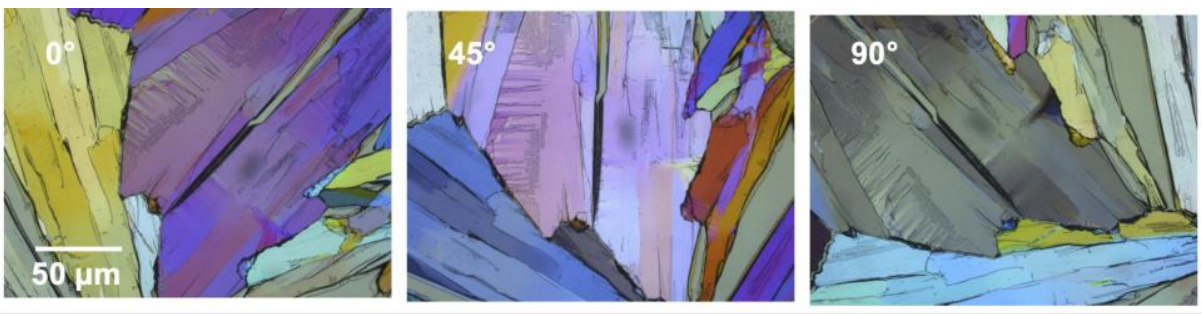

top

middle

bottom

B)
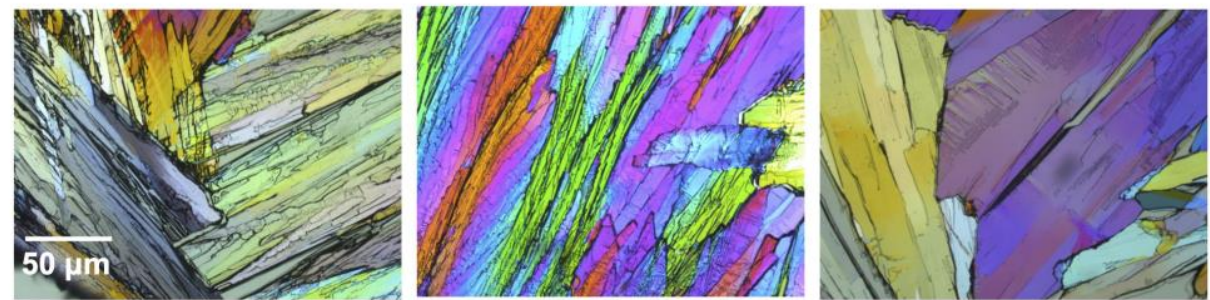

C)
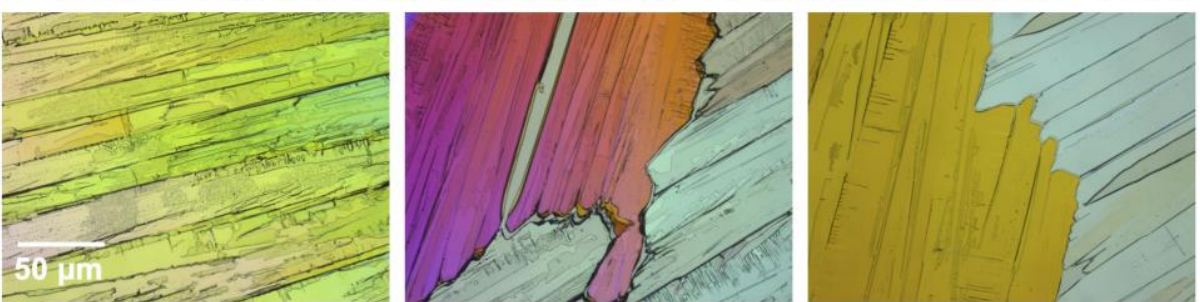

D)
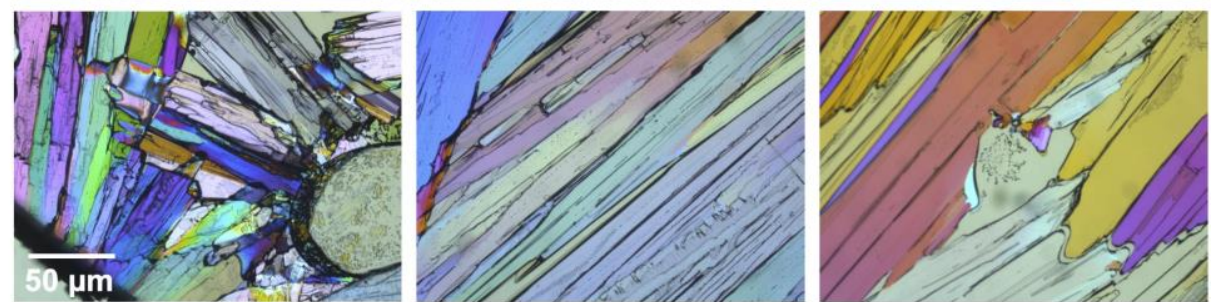

Figure S18. Polycrystalline films of dimethyl sulfone grown from the melt between two glass substrates exhibit crystals with an acicular crystal habit. (a) XPL micrographs were obtained of the same area of the polycrystalline film at $0^{\circ}, 45^{\circ}$, and $90^{\circ}$ to qualitatively demonstrate the crystallites are in different orientations. (b), (c), and (d) are XPL micrographs of three areas near the top, middle, and bottom of a sample along the center of the sample for three samples. 
A)
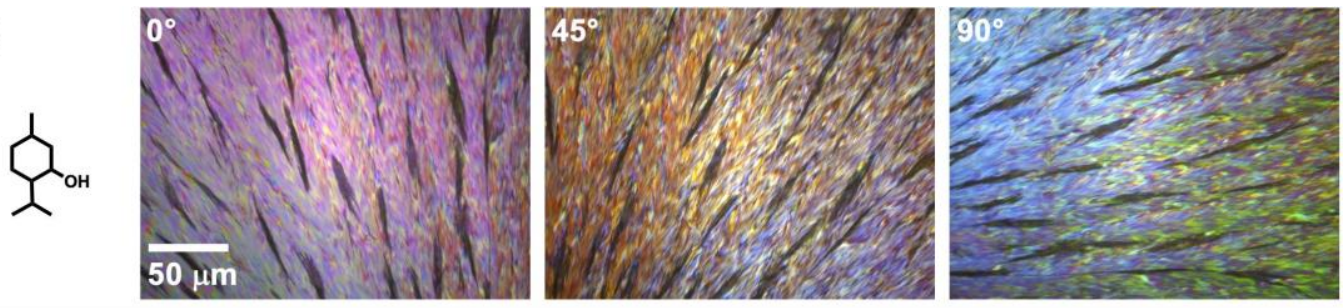

top

middle

bottom

B)
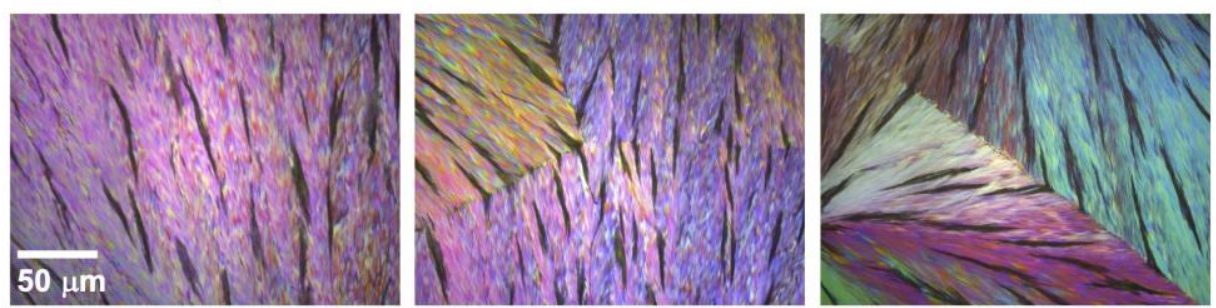

C)
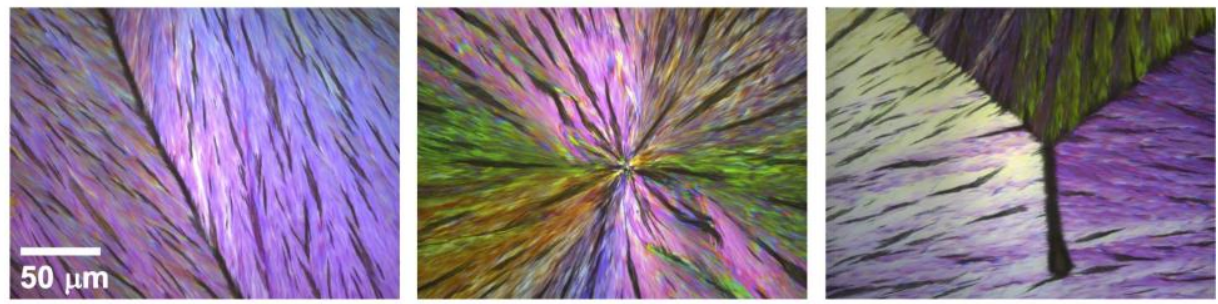

D)
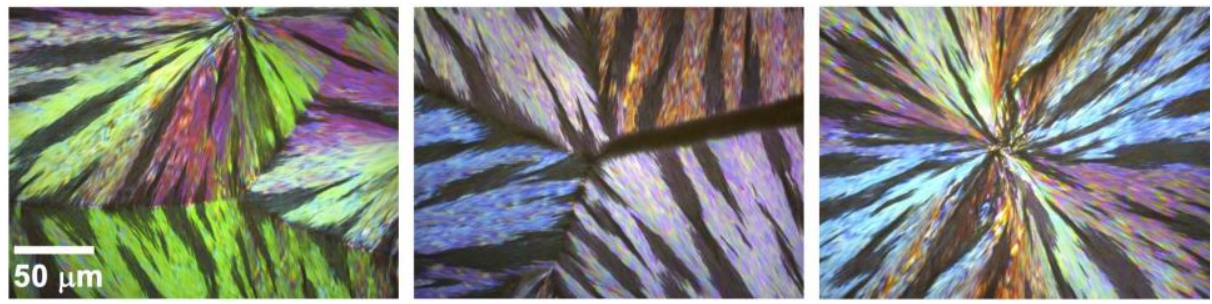

Figure S19. Polycrystalline films of (-)-menthol grown from the melt between two glass substrates exhibit crystals with an acicular crystal habit. (a) XPL micrographs were obtained of the same area of the polycrystalline film at $0^{\circ}, 45^{\circ}$, and $90^{\circ}$ to qualitatively demonstrate the crystallites are in different orientations. (b), (c), and (d) are XPL micrographs of three areas near the top, middle, and bottom of a sample along the center of the sample for three samples. 


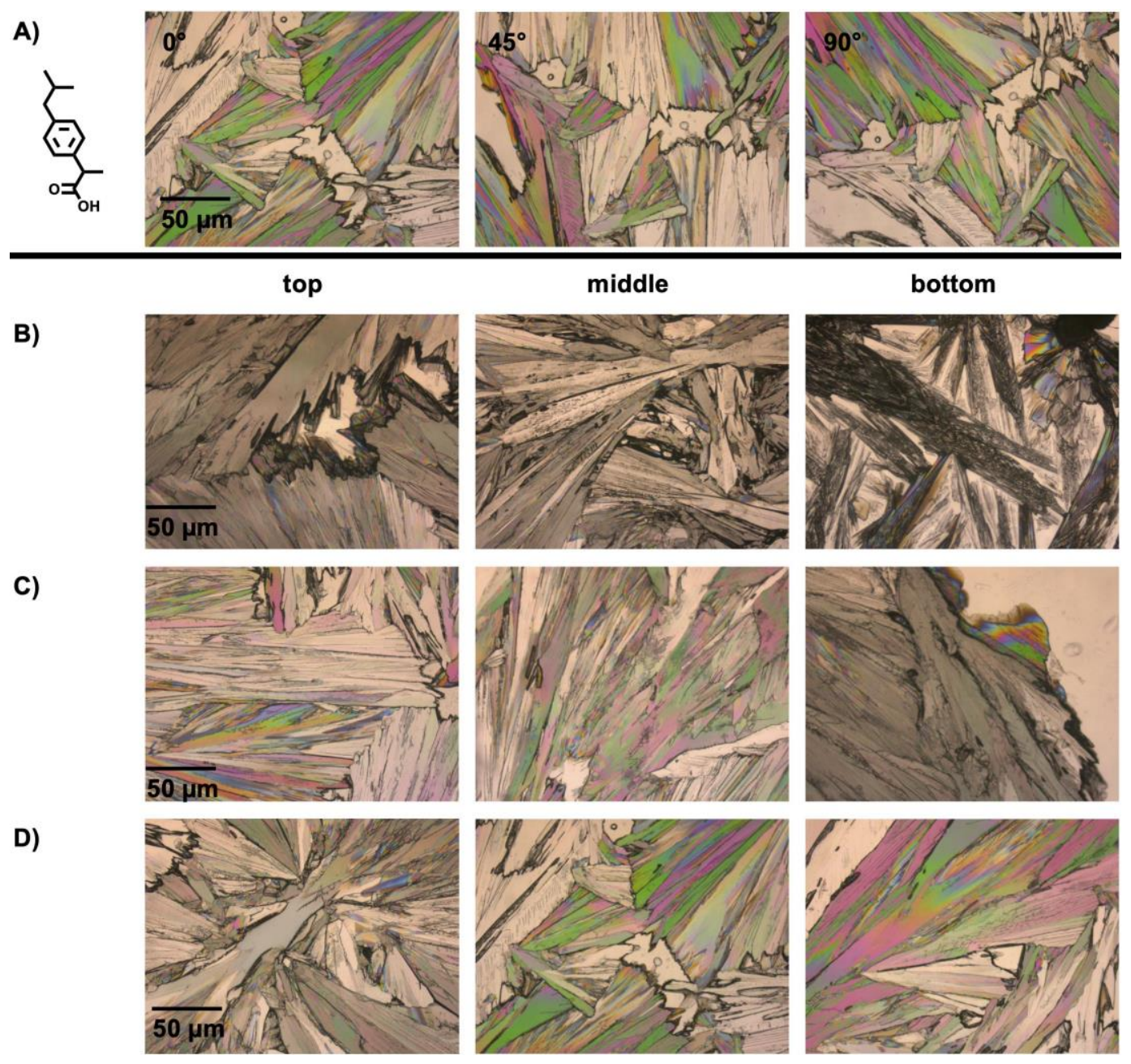

Figure S20. Polycrystalline films of (S)-ibuprofen grown from the melt between two glass substrates exhibit crystals with an acicular crystal habit. (a) XPL micrographs were obtained of the same area of the polycrystalline film at $0^{\circ}, 45^{\circ}$, and $90^{\circ}$ to qualitatively demonstrate the crystallites are in different orientations. (b), (c), and (d) are XPL micrographs of three areas near the top, middle, and bottom of a sample along the center of the sample for three samples. 


\section{SVI. Obtaining and Analyzing Optical Light Micrographs of Overlap Joints Before and After Shearing the Assembly}

Optical light (OL) micrographs were obtained for overlap joints before shearing the assemblies using an optical light microscope (Keyence High Definition Light Microscope).

OL micrographs were obtained for all assemblies of the initial ten assemblies that withstood typical handling. To increase contrast and visibility of the crystallites within the polycrystalline film, the light on the optical light microscope and an auxiliary light were used to illuminate assemblies. These lighting conditions assisted in discerning crystallite/crystallite interfaces (grain boundaries), transparent crystals, and voids. The auxiliary light was used to direct light at $a \sim 15^{\circ}$ angle to the surface of the assembly. The light from the optical light microscope directed light from above the sample, normal to the surface of the top substrate. A darkly tinted glass was placed behind the samples to minimize reflection of light. OL micrographs were obtained of the bottom and top substrates after fracture using the Keyence High Definition Light Microscope. The same lighting conditions were used for $\mathrm{OL}$ micrographs of the substrates from the sheared assembly. All OL micrographs obtained are organized by molecular solid and assembly in Figure S21-S28. 


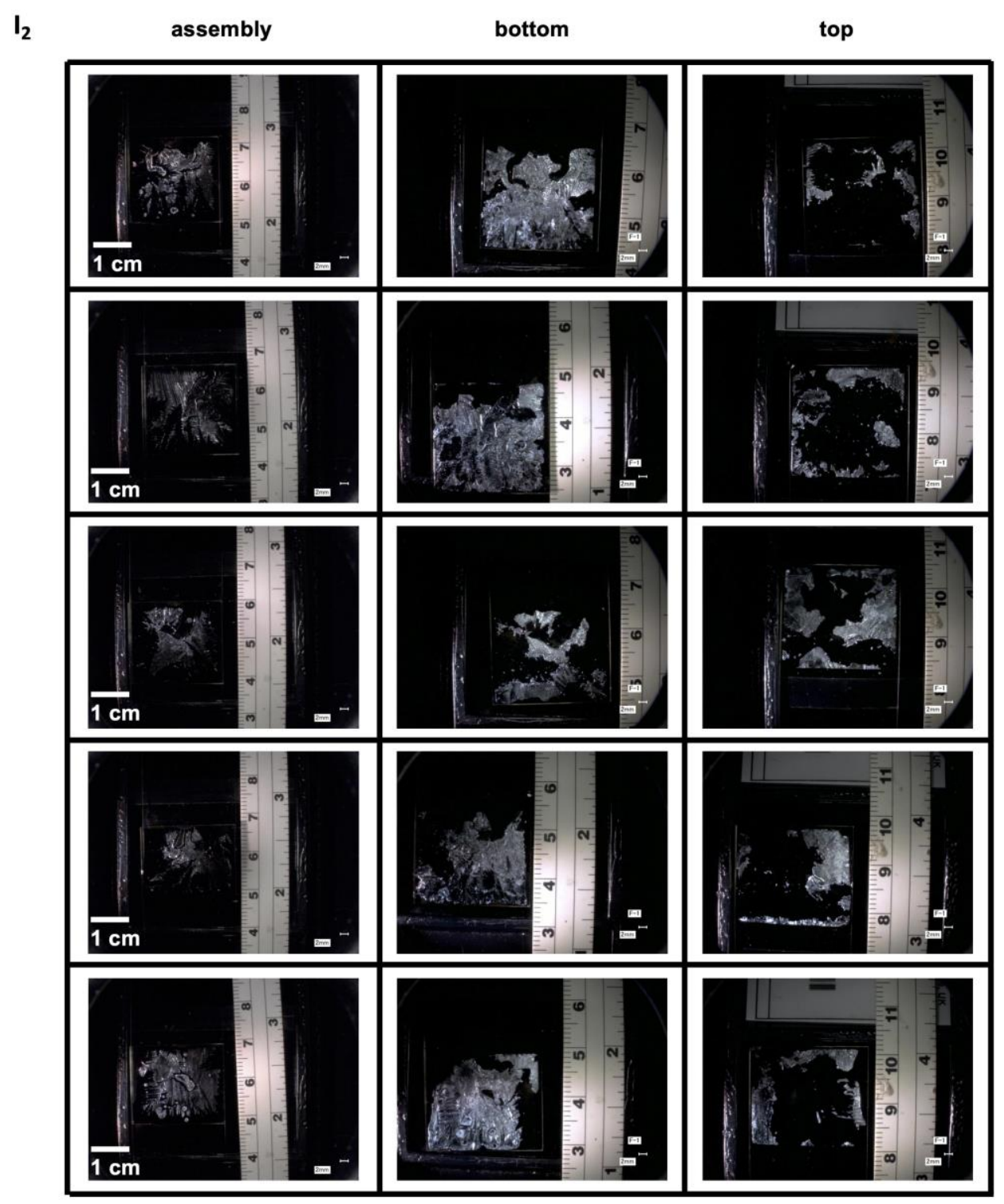

Figure S21. Optical micrographs of the bonded assembly with iodine as the sublimable adhesive before fracture and two halves after fracture were obtained to quantify the bonded area and the area of adhesive and cohesive fracture. 


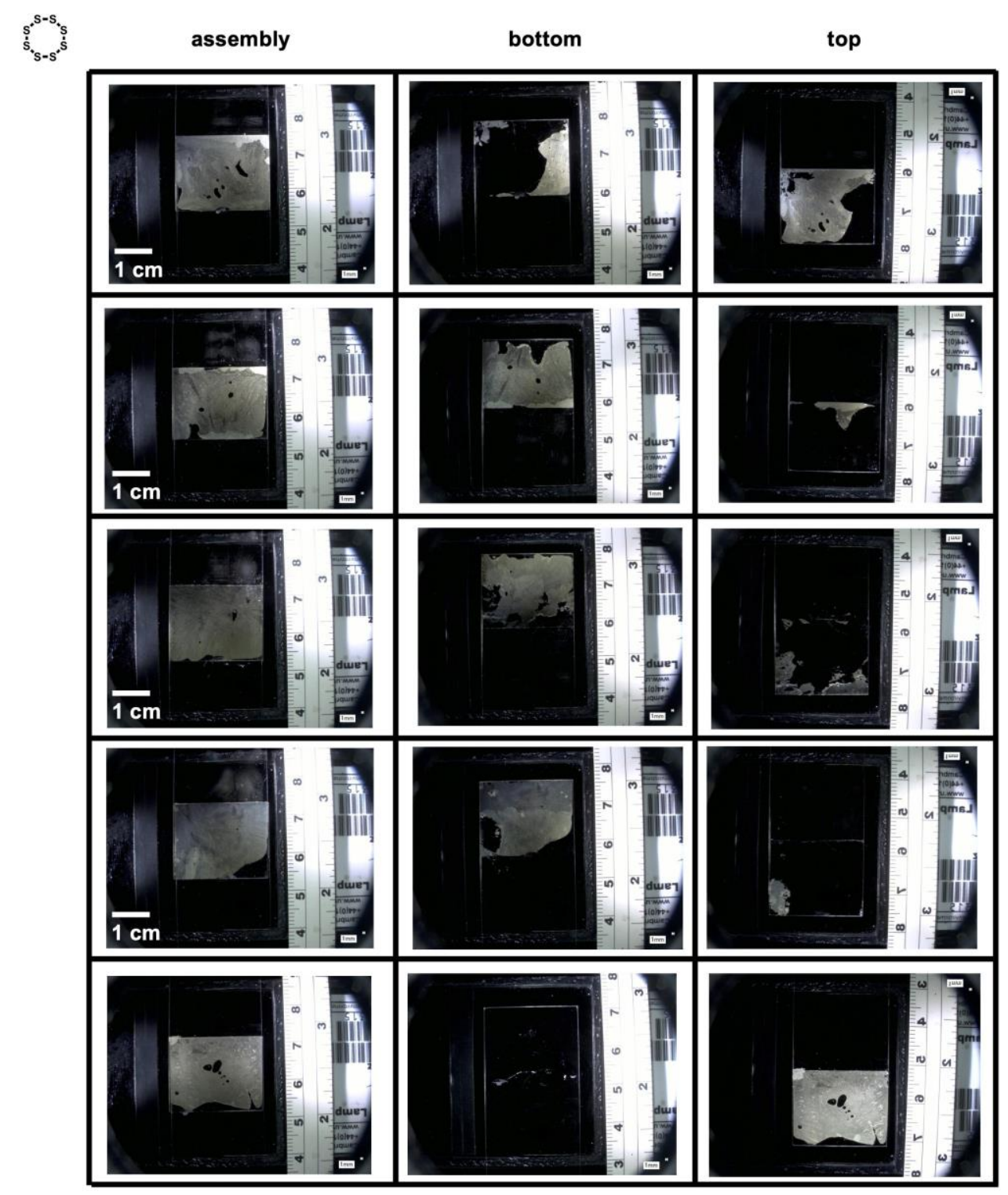

Figure S22. Optical micrographs of the bonded assembly with octacyclic sulfur as the sublimable adhesive before fracture and two halves after fracture were obtained to quantify the bonded area and the area of adhesive and cohesive fracture. 


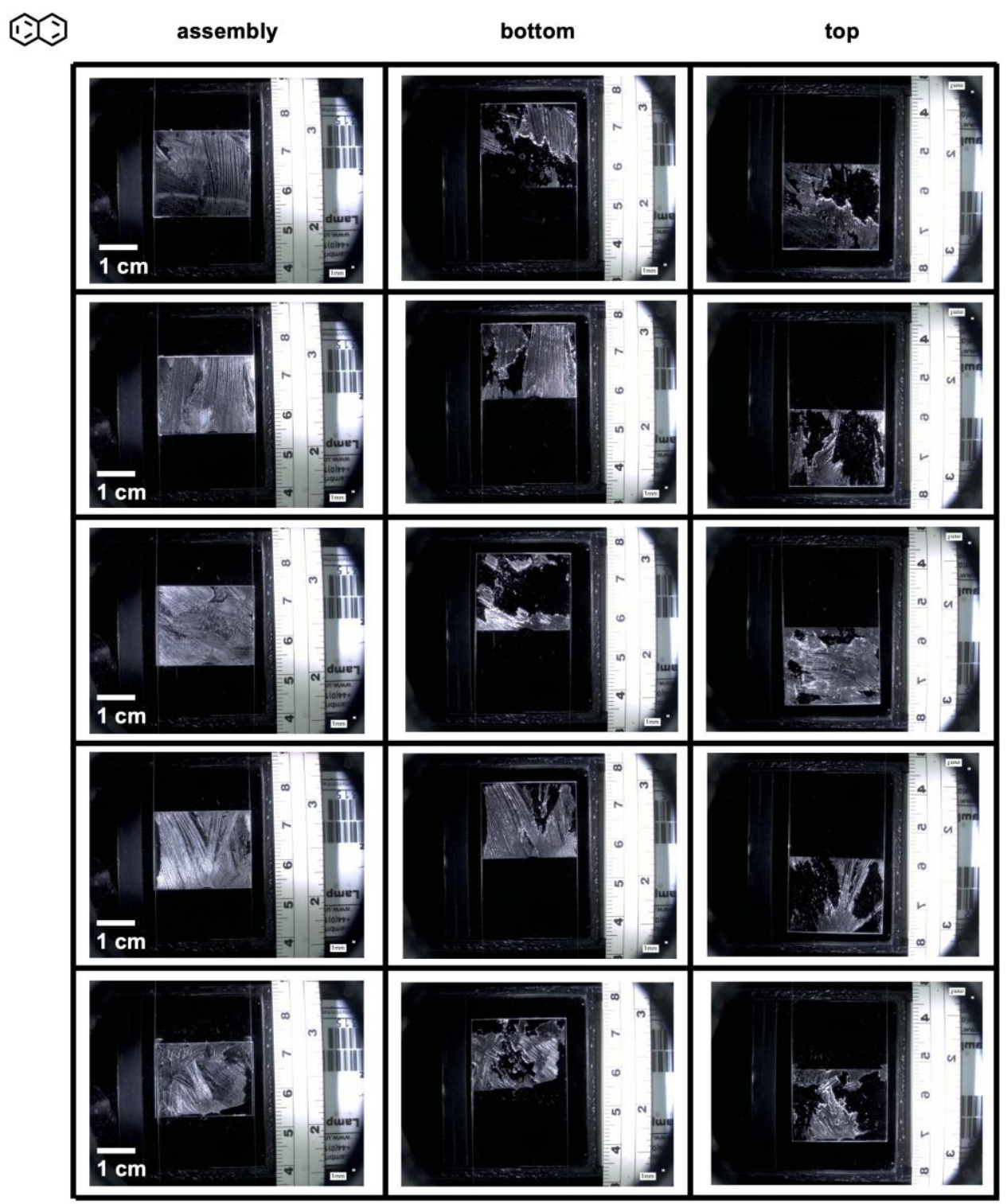

Figure S23. Optical micrographs of the bonded assembly with naphthalene as the sublimable adhesive before fracture and two halves after fracture were obtained to quantify the bonded area and the area of adhesive and cohesive fracture. 


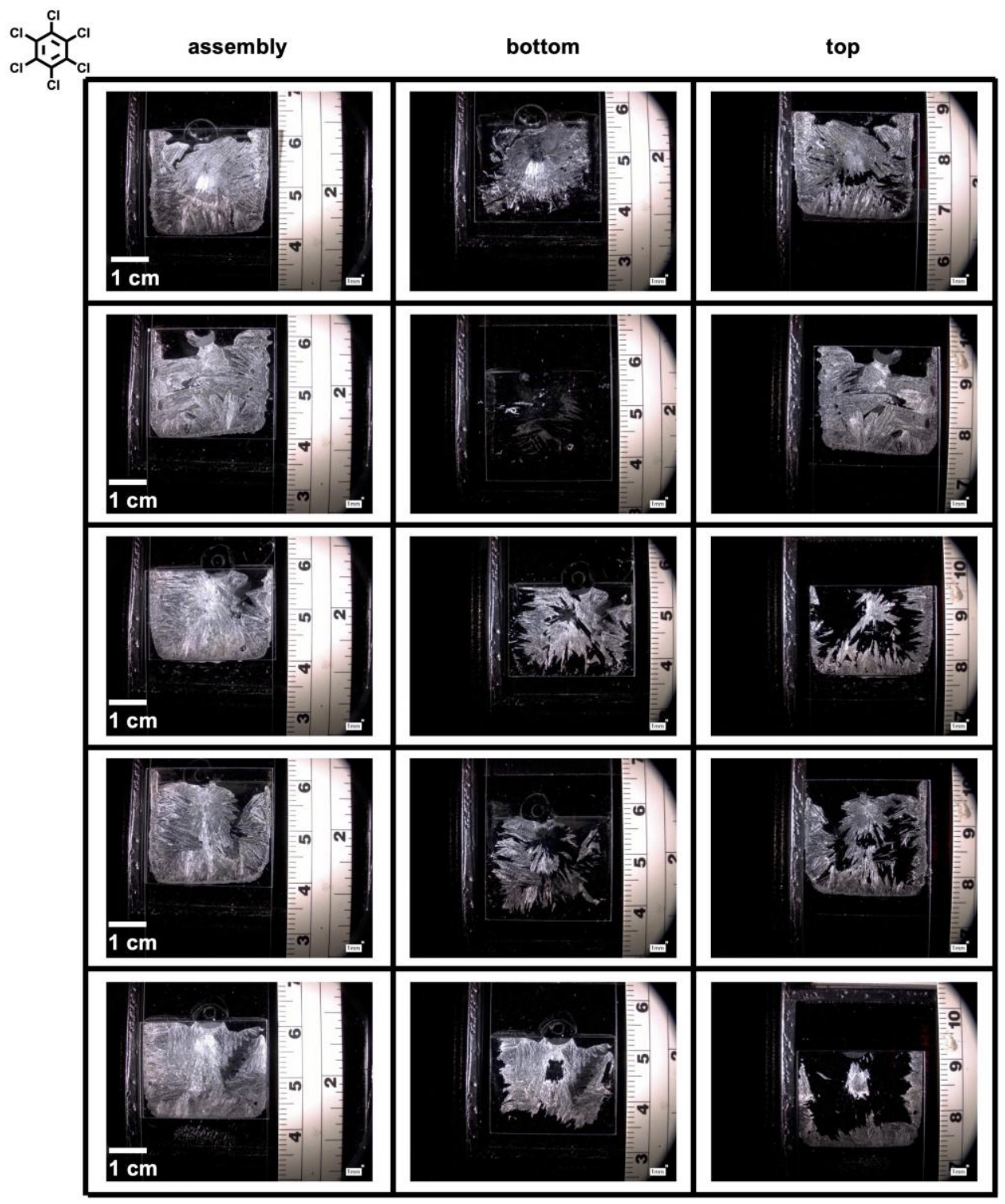

Figure S24. Optical micrographs of the bonded assembly with hexachlorobenzene as the sublimable adhesive before fracture and two halves after fracture were obtained to quantify the bonded area and the area of adhesive and cohesive fracture. 


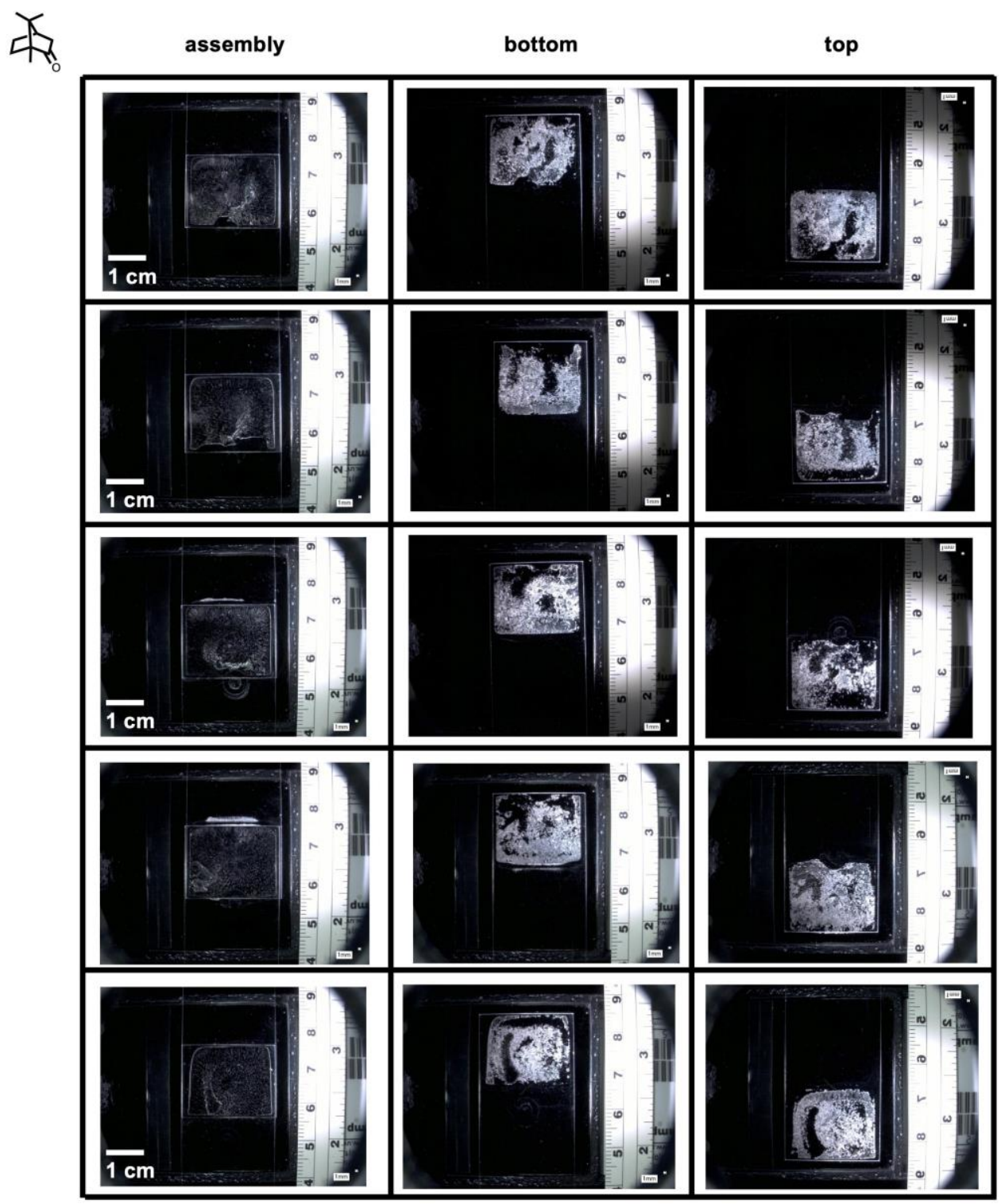

Figure S25. Optical micrographs of the bonded assembly with (+)-camphor as the sublimable adhesive before fracture and two halves after fracture were obtained to quantify the bonded area and the area of adhesive and cohesive fracture. 


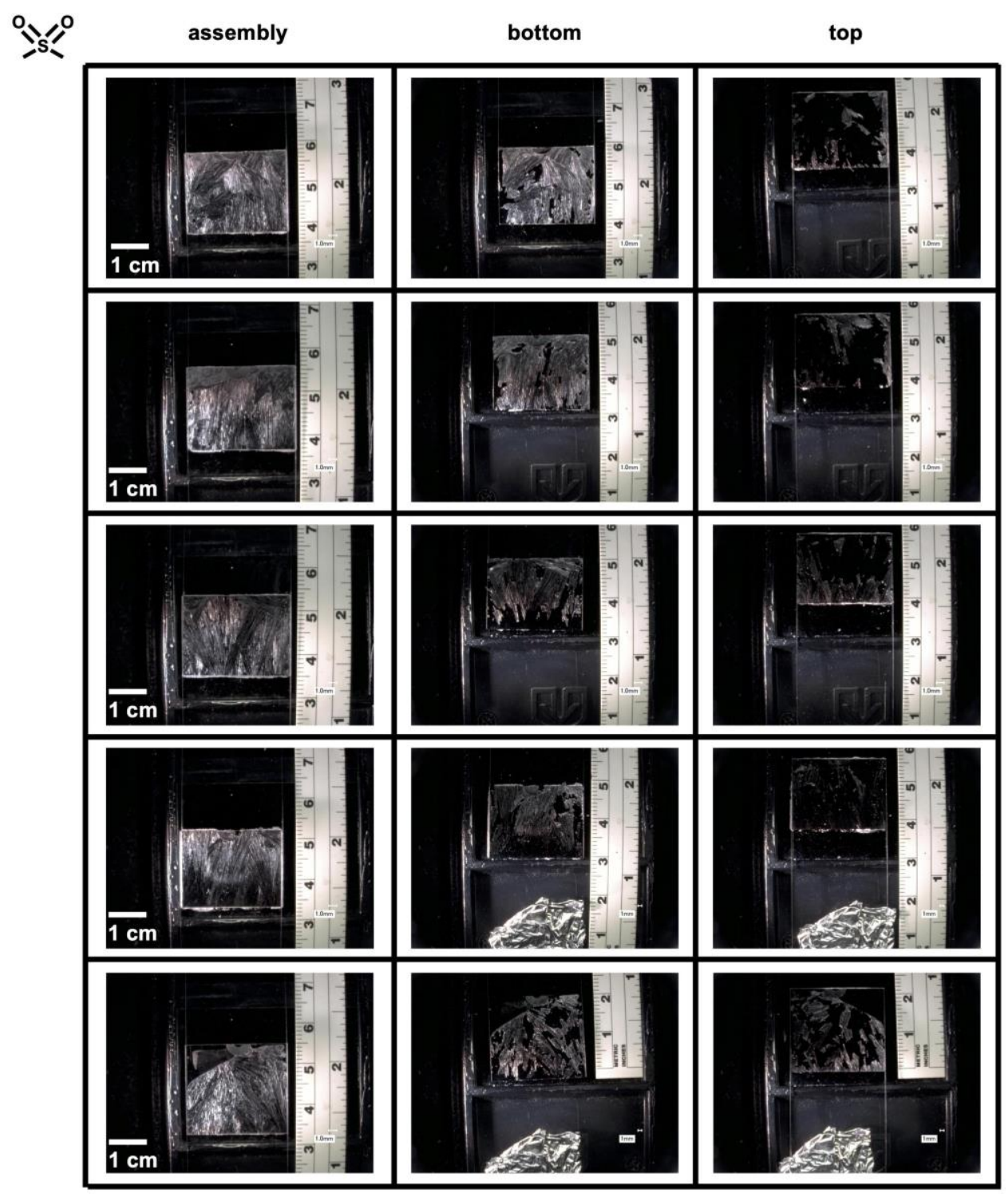

Figure S26. Optical micrographs of the bonded assembly with dimethyl sulfone as the sublimable adhesive before fracture and two halves after fracture were obtained to quantify the bonded area and the area of adhesive and cohesive fracture. 


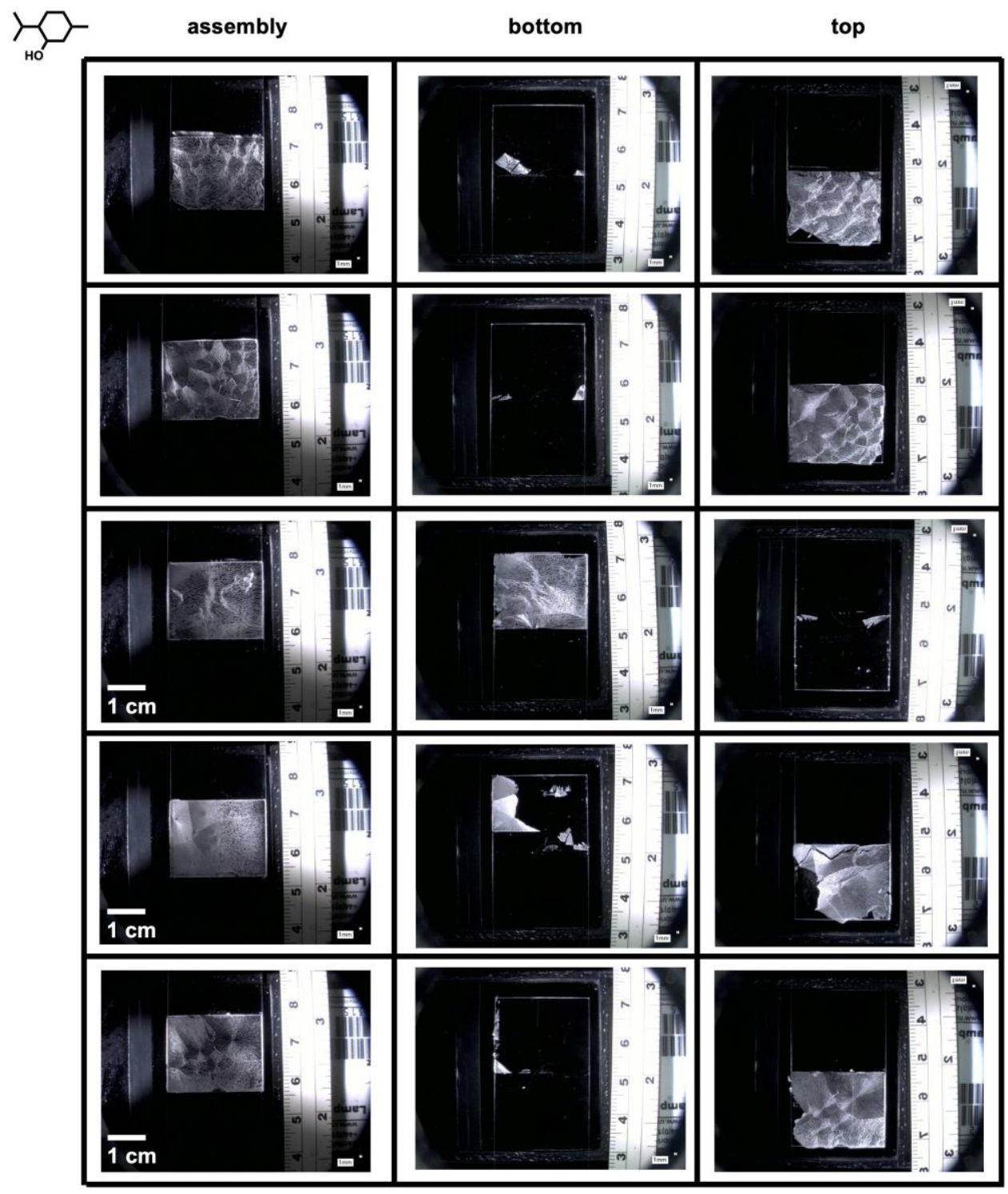

Figure S27. Optical micrographs of the bonded assembly with (-)-menthol as the sublimable adhesive before fracture and two halves after fracture were obtained to quantify the bonded area and the area of adhesive and cohesive fracture. 


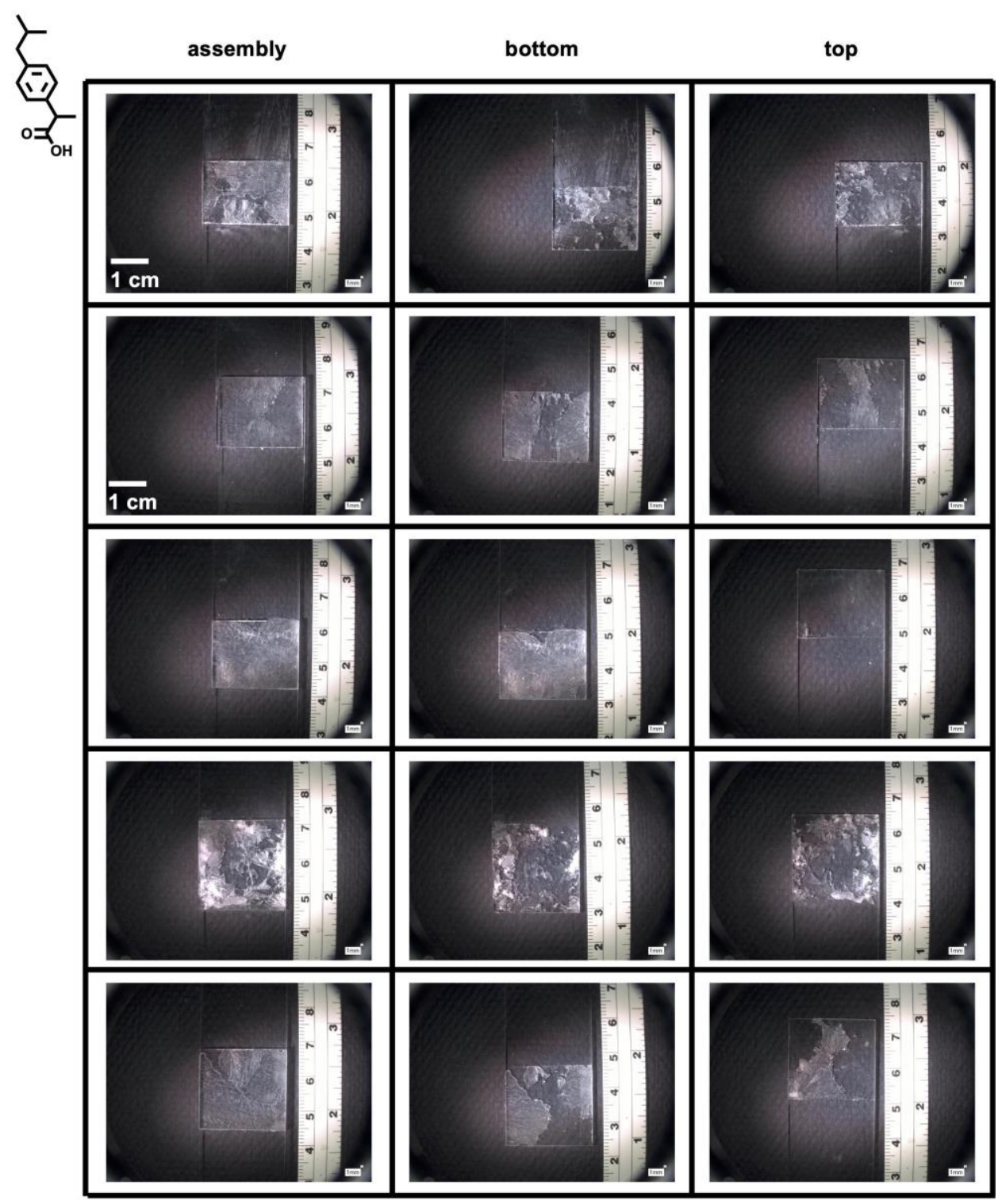

Figure S28. Optical micrographs of the bonded assembly with ibuprofen as the sublimable adhesive before fracture and two halves after fracture were obtained to quantify the bonded area and the area of adhesive and cohesive fracture. 
The surface area of the adhesive layer of the molecular solid between substrates was measured by processing the OL micrographs in Image J 5.0. The scale was set within the image. The Threshold plugin allowed for the contrast to be digitally optimized between crystallites and voids (transparent areas without molecular solid).

\section{SVII. Shear Testing of the Overlap Joint for Adhesive Measurements}

We employed an overlap shearing protocol similar to ASTM D-1002-10 to quantify the maximum force required to separate the two substrates melt-bonded by a molecular solid. ${ }^{\text {s29 }}$ Assemblies were placed into the clamps of the shear testing machine (Instron 5544 ) with the bottom substrate pointed up and sheared at a loading rate of $50 \mathrm{~N} \mathrm{~min}^{-1}$. The force and extension of the shear testing was recorded using Blue Hill software, exported as comma-separated value files, and analyzed in Microsoft Excel. The maximum force is the highest value of force withstood by the overlap joint before failing, separating into two substrates. The maximum extension is the extension value at the maximum force. An instrumental artifact, which arises as slack is taken-up from the mechanical testing rig, was subtracted from the maximum extension to obtain the accurate extension of the overlap joint. To account for variation in the area of the adhesive layer in the overlap joint we introduced the parameter $\tau(\mathrm{kPa})$ as the ratio of the maximum force $(\mathrm{N})$ withstood before the overlap joint failed, to the area of the adhesive layer $\left(\mathrm{cm}^{2}\right)$ in the overlap joint (Eq. S2). To account for variation in the thickness of the adhesive layer we introduced the parameter $\gamma$ as the ratio of the extension of the overlap joint $(\mathrm{cm})$, to the thickness of the adhesive layer (cm) (Eq. S3). 


$$
\begin{gathered}
\tau\left(N \mathrm{~cm}^{-2}\right)=\frac{\text { maximum force before failure }(N)}{\text { surface area of adhesive layer }\left(\mathrm{cm}^{2}\right)} \\
\gamma\left(\mathrm{m} \mathrm{m}^{-1}\right)=\frac{\text { extension of the overlap joint }(\mathrm{cm})}{\text { thickness of the adhesive layer }(\mathrm{cm})}
\end{gathered}
$$

\section{SVIII. Quantifying the Shear Moduli of Adhesives in Overlap Joints}

All samples were assumed to exhibit brittle fracture mechanics. The shear modulus of each sample (i.e., the stress to strain ratio) was measured by calculating the slope of the linear region of the stress-strain profile. The individual modulus of each sample was calculated independently.

\section{SIX. Micrographs Obtained by White Light Interferometry}

The topography of the surface of the fractured adhesive layer was characterized by White Light Interferometry (WLI) microscopy using a WLI microscope (Zygo NewView 5000 3D Surface Profiler). The WLI micrographs were obtained from three scans averaged with scan length of $145 \mu \mathrm{m}$ in the preset "Micro" application settings of the Zygo Mx software. The bottom substrate was selected unless there was an insufficient area of adhesive on the substrate to characterize or be representative of how the majority of the adhesive layered fractured. Micrographs were obtained at the edge and surface of the fractured adhesive layer to ensure a holistic characterization of the surfaces generated by fracture. Representative micrographs are presented in Figures S29-S36. 

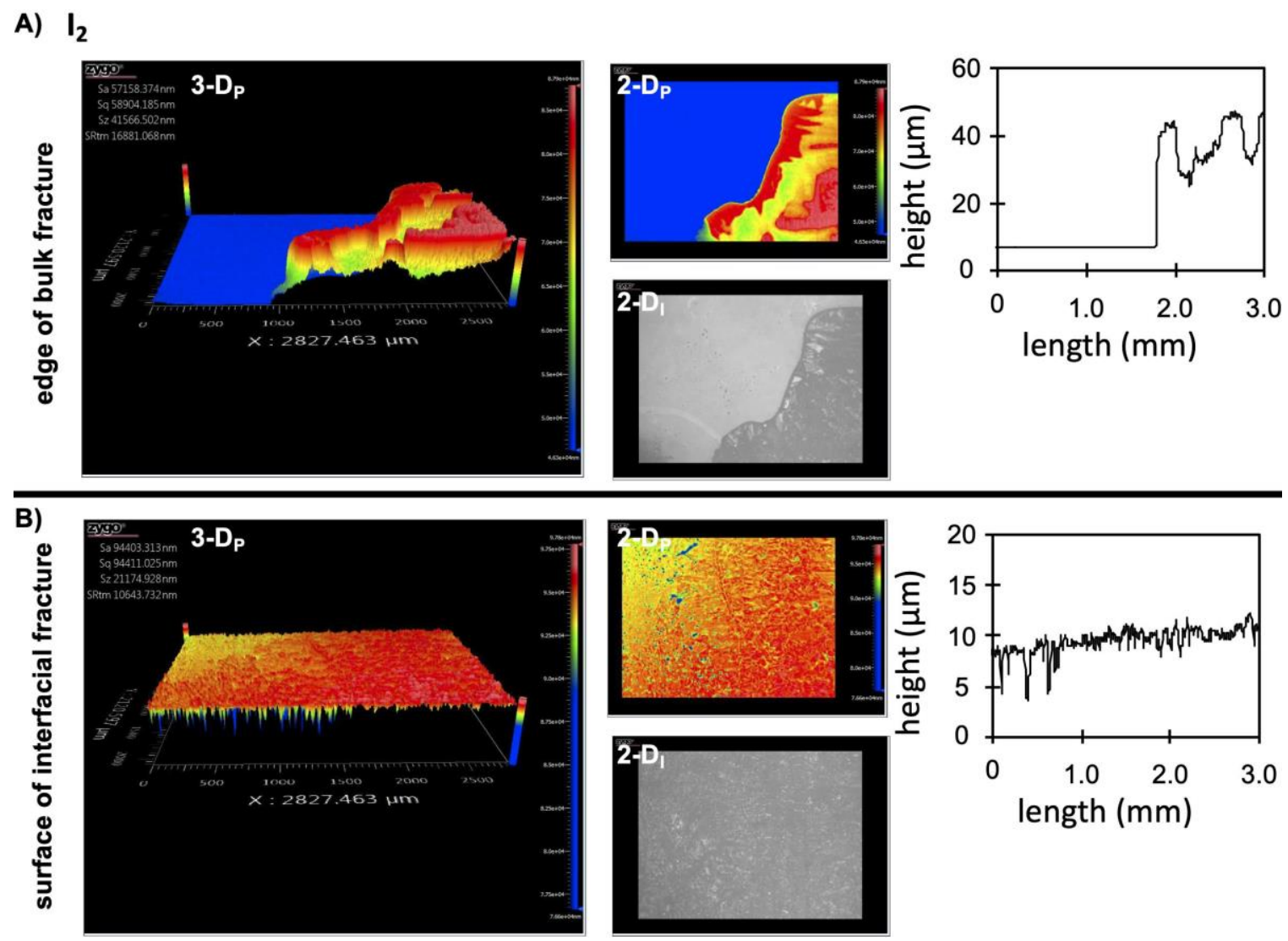

Figure S29. Micrographs obtained by White Light Interferometry at the edge and surface of a fracture through a polycrystalline film of iodine melt-bonded between two glass substrates. The edge and surface are generated from a fracture through the bulk of the polycrystalline film and interface between the polycrystalline film and glass, respectively. A 3-D profile (3- $\left.D_{P}\right), 2-D$ profile $\left(2-D_{P}\right), 2-D$ image $\left(2-D_{1}\right)$ and plot (far right) are generated for each WLI micrograph of the edge (a) and surface (b). The lowest and highest surfaces are represented by blue and red, respectively, in the 3-Dp and 2-Dp. The plot for the height of surface fractured from the interface at the glass does not represent the total height of the film. 


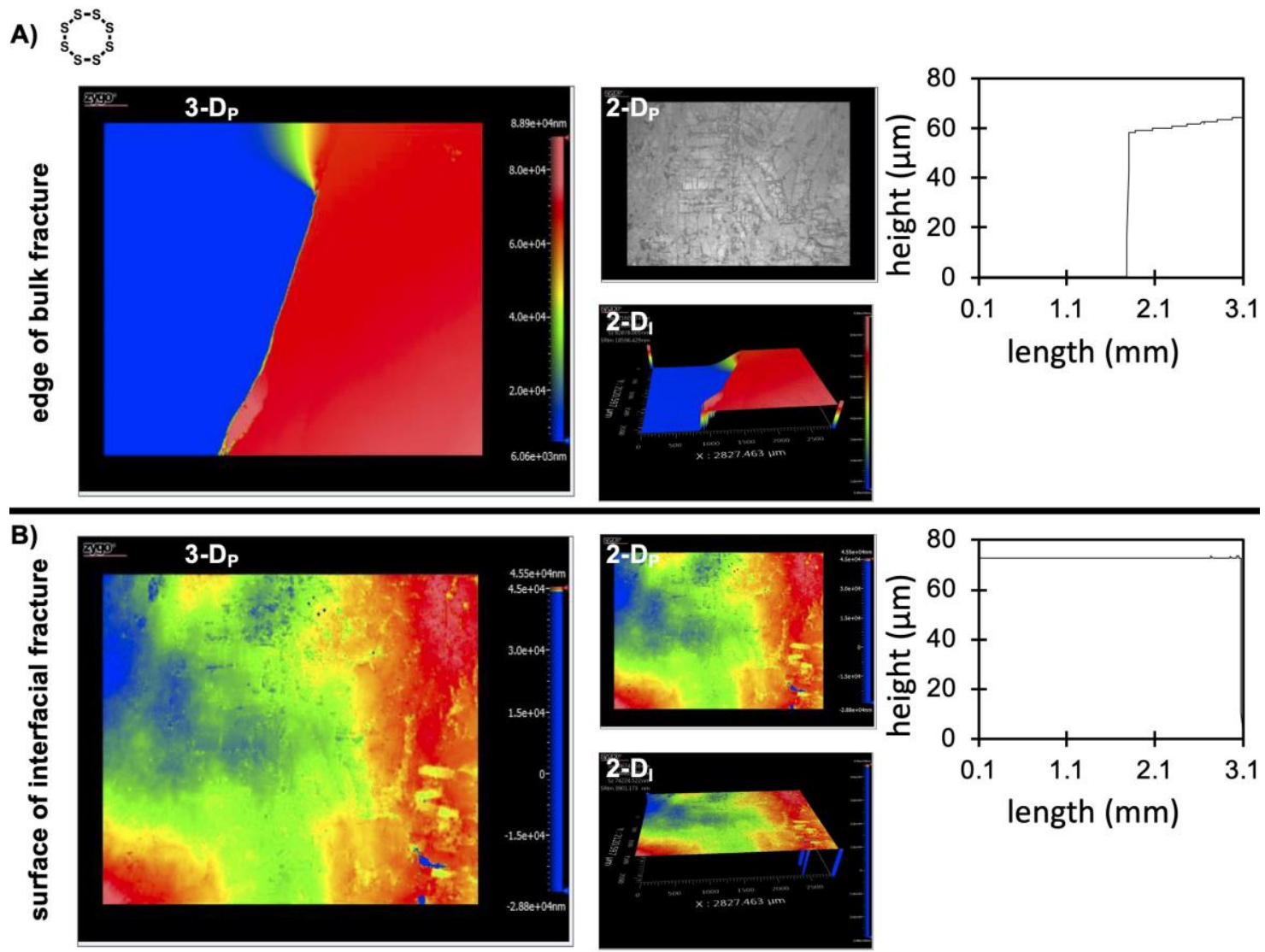

Figure S30. Micrographs obtained by White Light Interferometry at the edge and surface of a fracture through a polycrystalline film of octacyclic sulfur melt-bonded between two glass substrates. The edge and surface are generated from a fracture through the bulk of the polycrystalline film and interface between the polycrystalline film and glass, respectively. A 3-D profile (3- $\left.D_{P}\right)$, 2-D profile (2-D $)$, 2-D image $\left(2-D_{1}\right)$ and plot (far right) are generated for each WLI micrograph of the edge (a) and surface (b). The lowest and highest surfaces are represented by blue and red, respectively, in the 3-Dp and 2-Dp. The plot for the height of surface fractured from the interface at the glass does not represent the total height of the film. 

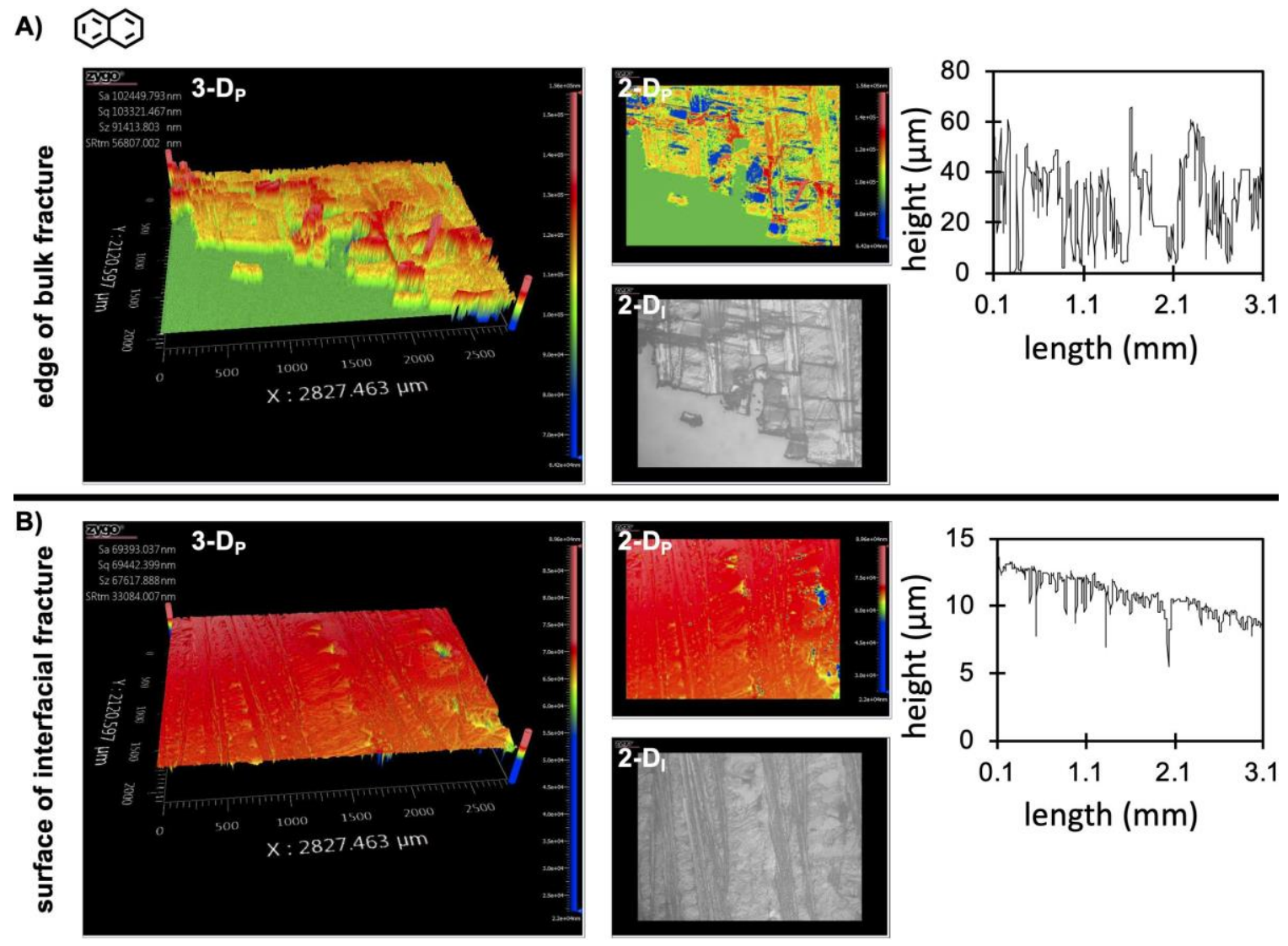

Figure S31. Micrographs obtained by White Light Interferometry at the edge and surface of a fracture through a polycrystalline film of naphthalene melt-bonded between two glass substrates. The edge and surface are generated from a fracture through the bulk of the polycrystalline film and interface between the polycrystalline film and glass, respectively. A 3-D profile (3-DP), 2-D profile (2-DP), 2-D image (2-D।) and plot (far right) are generated for each WLI micrograph of the edge (a) and surface (b). The lowest and highest surfaces are represented by blue and red, respectively, in the 3-Dp and 2-Dp. The plot for the height of surface fractured from the interface at the glass does not represent the total height of the film. 


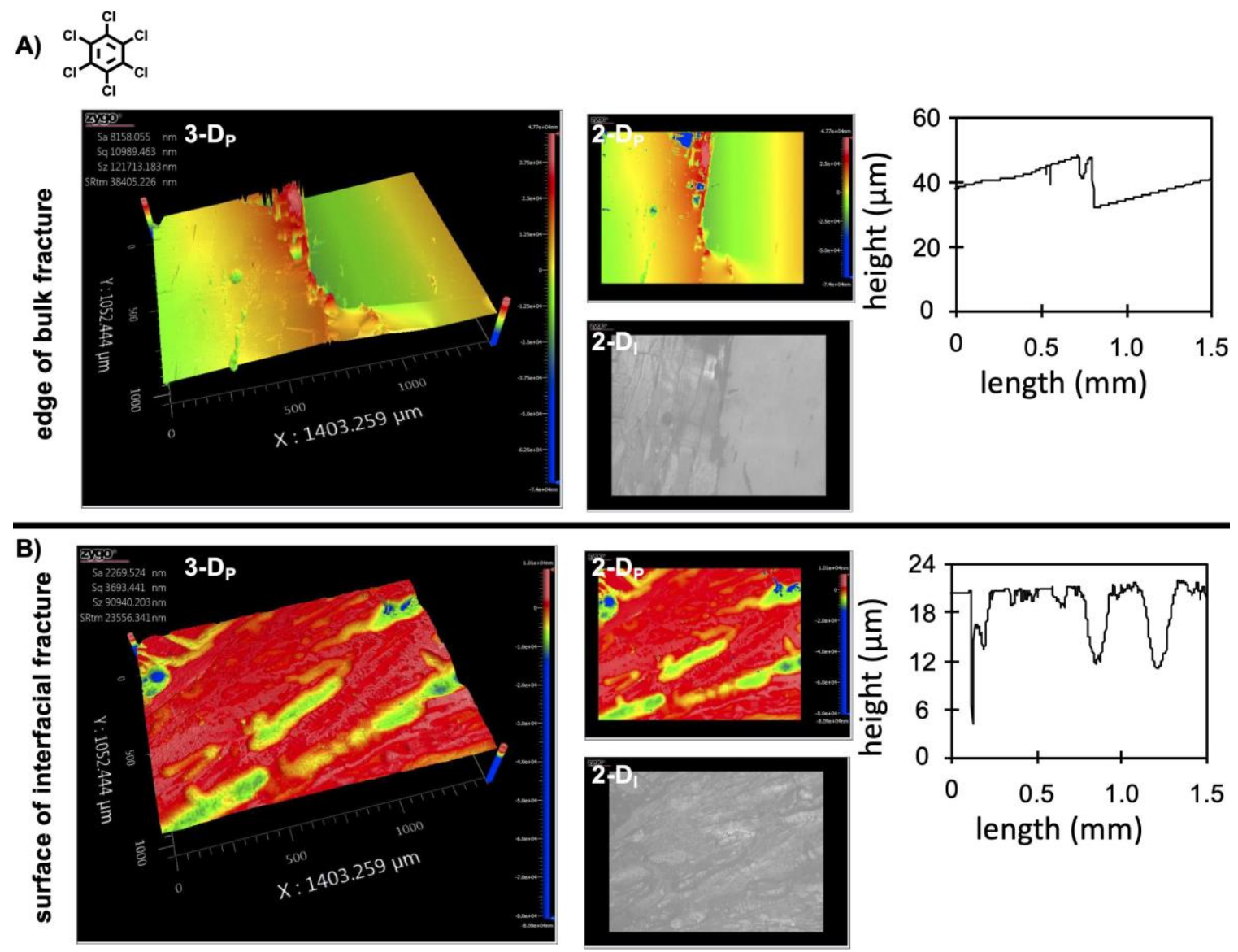

Figure S32. Micrographs obtained by White Light Interferometry at the edge and surface of a fracture through a polycrystalline film of hexachlorobenzene meltbonded between two glass substrates. The edge and surface are generated from a fracture through the bulk of the polycrystalline film and interface between the polycrystalline film and glass, respectively. A 3-D profile (3-DP), 2-D profile (2-DP), 2-D image $\left(2-D_{1}\right)$ and plot (far right) are generated for each WLI micrograph of the edge (a) and surface (b). The lowest and highest surfaces are represented by blue and red, respectively, in the 3-Dp and 2-Dp. The plot for the height of surface fractured from the interface at the glass does not represent the total height of the film. 


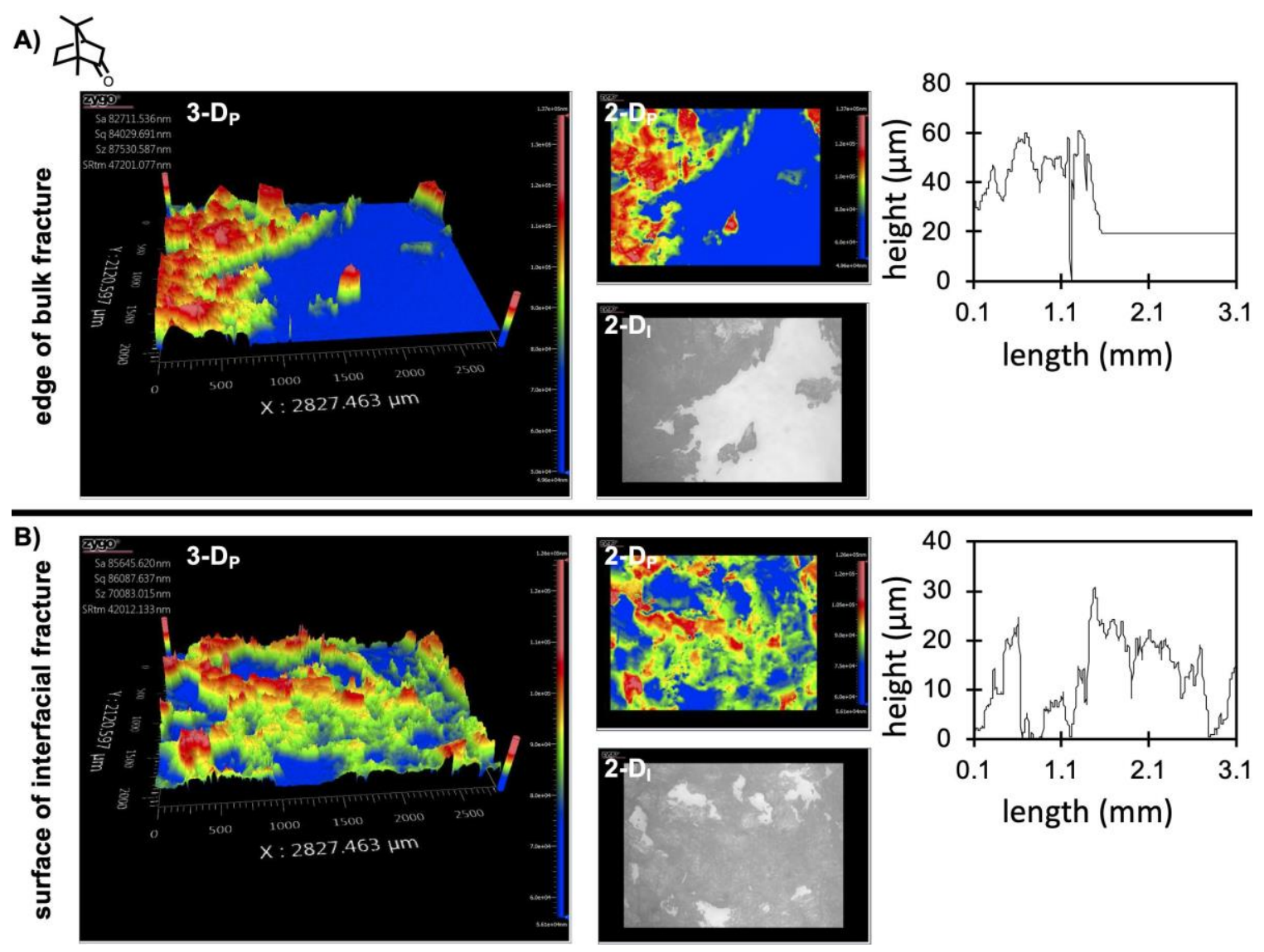

Figure S33. Micrographs obtained by White Light Interferometry at the edge and surface of a fracture through a polycrystalline film of $(+)$-camphor melt-bonded between two glass substrates. The edge and surface are generated from a fracture through the bulk of the polycrystalline film and interface between the polycrystalline film and glass, respectively. A 3-D profile (3- $\left.D_{P}\right), 2-D$ profile $\left(2-D_{P}\right), 2-D$ image $\left(2-D_{1}\right)$ and plot (far right) are generated for each WLI micrograph of the edge (a) and surface (b). The lowest and highest surfaces are represented by blue and red, respectively, in the 3-Dp and 2-Dp. The plot for the height of surface fractured from the interface at the glass does not represent the total height of the film. 

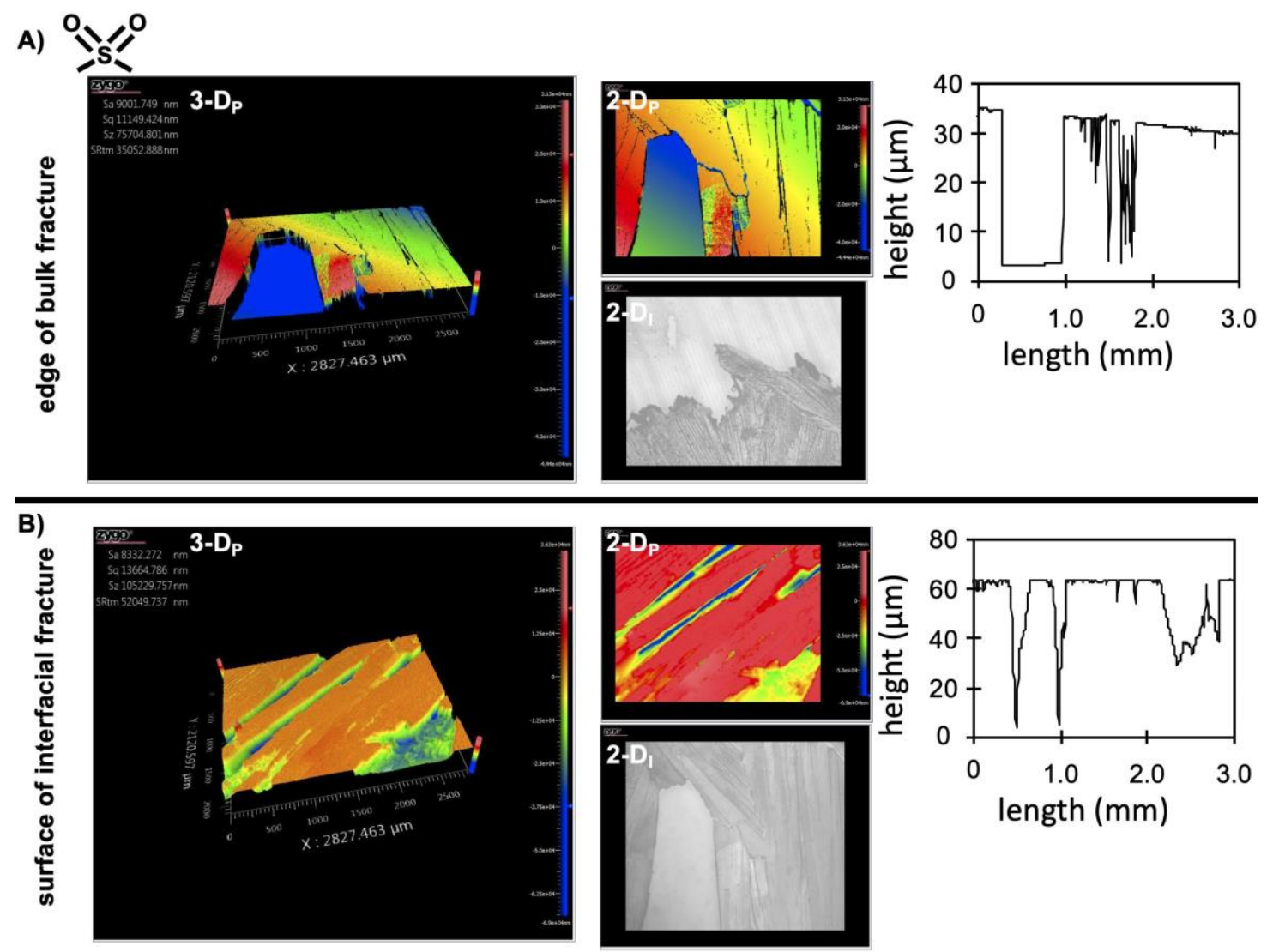

Figure S34. Micrographs obtained by White Light Interferometry at the edge and surface of a fracture through a polycrystalline film of dimethyl sulfone melt-bonded between two glass substrates. The edge and surface are generated from a fracture through the bulk of the polycrystalline film and interface between the polycrystalline film and glass, respectively. A 3-D profile (3-DP), 2-D profile (2-DP), 2-D image (2-D। $)$ and plot (far right) are generated for each WLI micrograph of the edge (a) and surface (b). The lowest and highest surfaces are represented by blue and red, respectively, in the 3-Dp and 2-Dp. The plot for the height of surface fractured from the interface at the glass does not represent the total height of the film. 


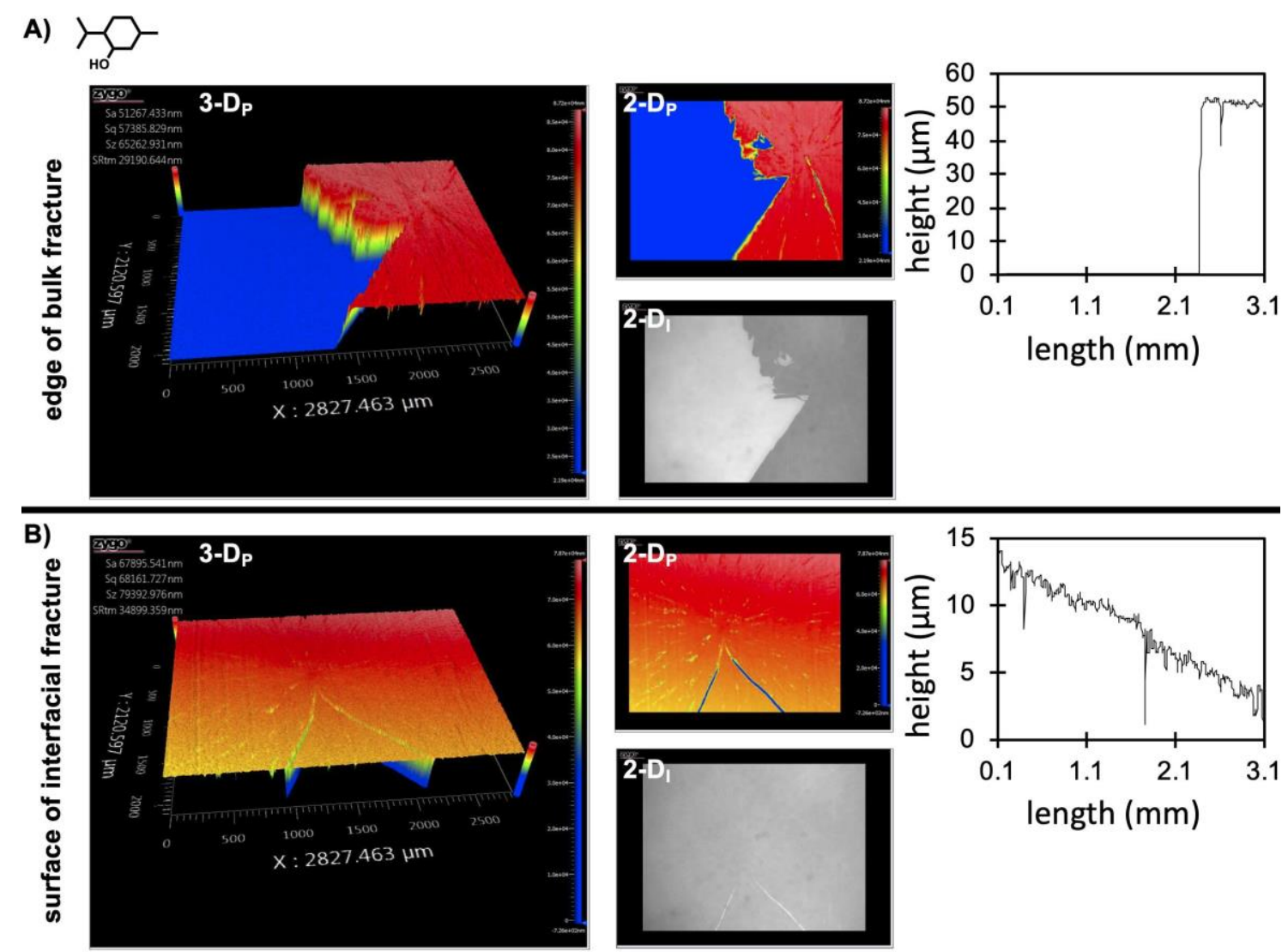

Figure S35. Micrographs obtained by White Light Interferometry at the edge and surface of a fracture through a polycrystalline film of (-)-menthol melt-bonded between two glass substrates. The edge and surface are generated from a fracture through the bulk of the polycrystalline film and interface between the polycrystalline film and glass, respectively. A 3-D profile (3-DP), 2-D profile (2-DP), 2-D image (2- $\left.D_{1}\right)$ and plot (far right) are generated for each WLI micrograph of the edge (a) and surface (b). The lowest and highest surfaces are represented by blue and red, respectively, in the 3-Dp and 2-Dp. The plot for the height of surface fractured from the interface at the glass does not represent the total height of the film. 


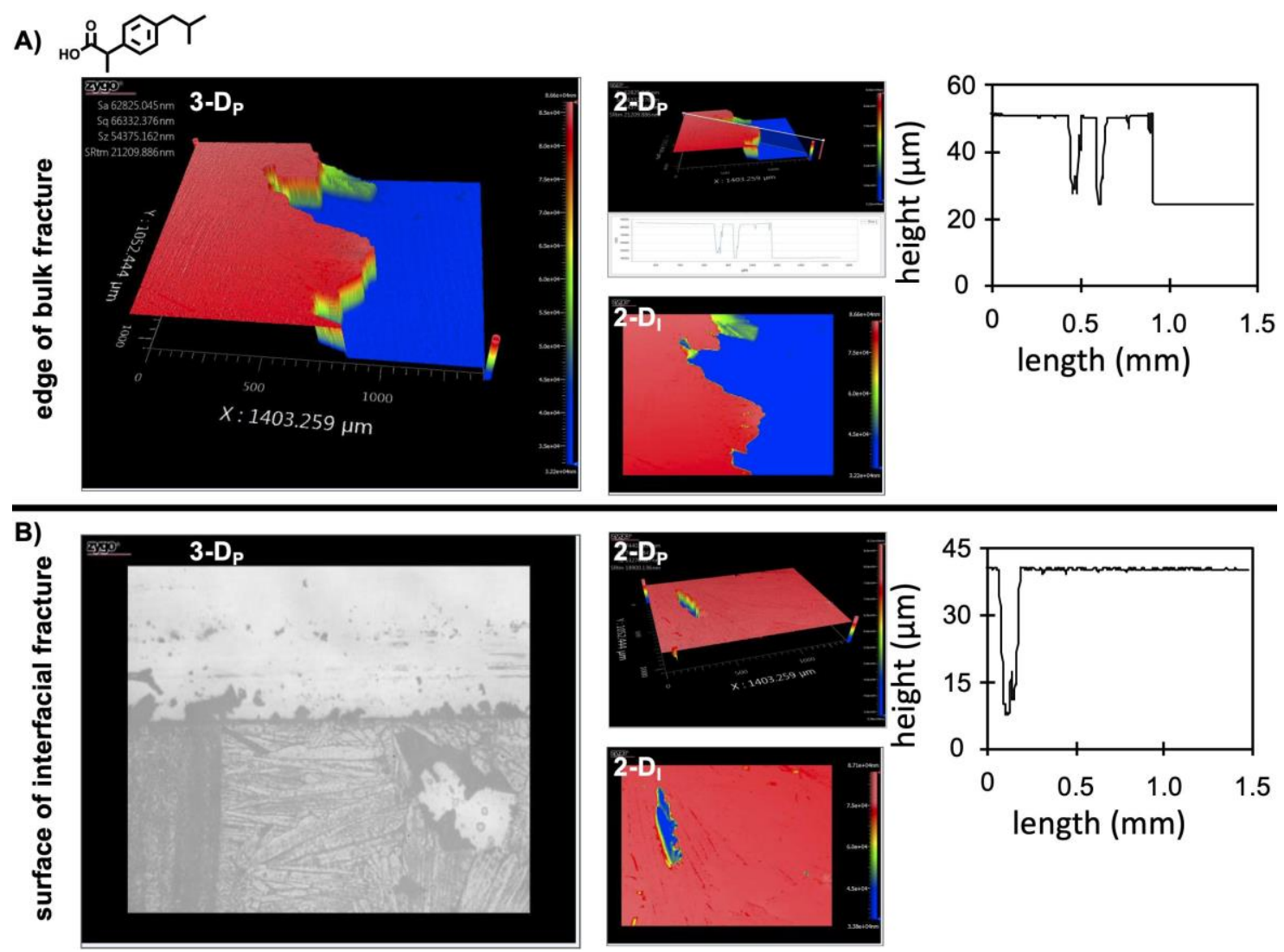

Figure S36. Micrographs obtained by White Light Interferometry at the edge and surface of a fracture through a polycrystalline film of ibuprofen melt-bonded between two glass substrates. The edge and surface are generated from a fracture through the bulk of the polycrystalline film and interface between the polycrystalline film and glass, respectively. A 3-D profile (3-DP), 2-D profile (2-DP), 2-D image (2- $\left.D_{1}\right)$ and plot (far right) are generated for each WLI micrograph of the edge (a) and surface (b). The lowest and highest surfaces are represented by blue and red, respectively, in the 3-Dp and 2-Dp. The plot for the height of surface fractured from the interface at the glass does not represent the total height of the film. 


\section{SX. Powder X-Ray Diffraction}

We collected spectra using a Rigaku MiniFlex Powder X-ray Diffractometer (PXRD) equipped with a Cu $600 \mathrm{~W}\left(40 \mathrm{kV}, 15 \mathrm{~mA}, \lambda=1.54 \AA\right.$ ) radiation source. The range between $2^{\circ}$ and $60^{\circ} 2 \theta$ was scanned, with a step size of $0.02^{\circ}$ per 2 seconds. Commercially obtained, finely ground molecular solids were used as the reference bulk samples, assuming random orientation of crystallites within the sample. We accept that the distribution of Bragg planes observed for the reference bulk samples is skewed because crystallite packing is determined by their morphology and size, so the randomness of crystallite distribution is skewed due to the preferred cleavage planes of the molecular solids. Single crystal X-ray diffraction data files (CIFs) were used to generate simulated PXRD spectra using Mercury 3.3. Glass slides were used to melt-bond adherends using the molecular solids and then sheared apart. The remaining film on the surface of the adherend was used to obtain diffraction patterns of each adhesive.

The peak intensity for a given $2 \theta$ is predominantly determined by the number of X-rays that diffract along the corresponding Bragg plane and reach the detector through constructive interference. ${ }^{530}$ The relative peak intensity percentages for the dominant Bragg planes of polycrystalline films were calculated using Equation S4. Four peaks with the highest intensity in the melt-bonded assembly were selected to represent the total peak intensity of the assembly. If the PXRD spectra of the melt-bonded assembly has less than four obvious peaks, the remaining peaks required are selected from the bulk. The maximum intensity of each peak was used to represent the entire peak. The intensity of a selected peak was divided by the total peak intensity of the sample to produce the relative peak intensity (Eq. S4). Differences in the relative peak 
intensities of the bulk and assemblies were interpreted as the crystallites taking on preferred orientation from melt-bonding.

$$
\text { relative peak intensity }(\%)=\frac{\text { intensity of selected peak }}{\text { sum intensity of all peaks }}
$$



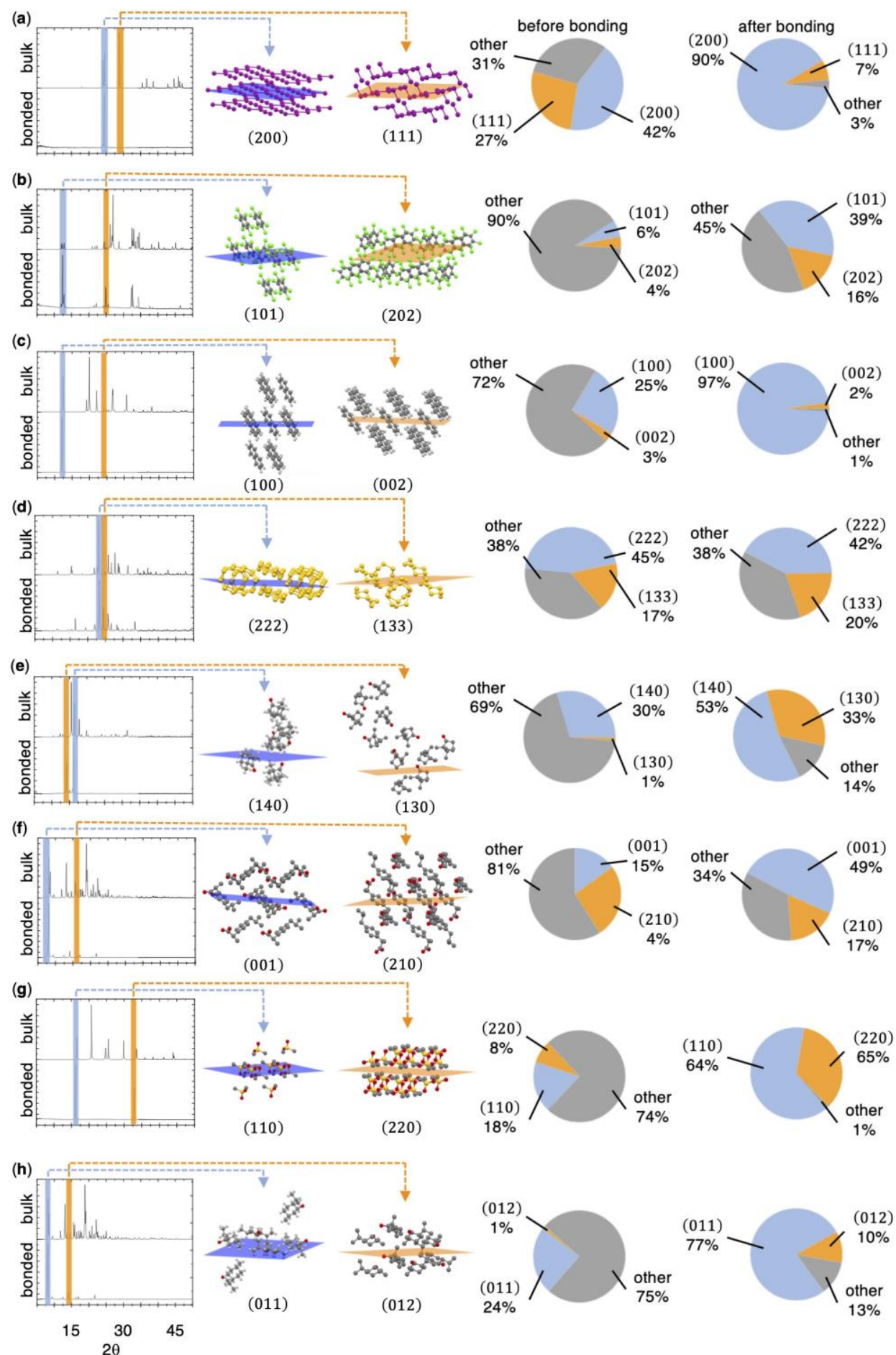

Figure S37. X-ray diffraction of polycrystalline films reveals that melt-bonding between glass substrates induces preferential orientation. Preferential orientation is evident in the reduction in the number of Bragg peaks in the melt-bonded samples relative to bulk samples in (a) iodine, (b) hexachlorobenzene, (c) naphthalene, (d) octacyclic sulfur, (e) (+)-camphor, (f) (-)-menthol, (g) dimethyl sulfone, and (h) Sibuprofen. 


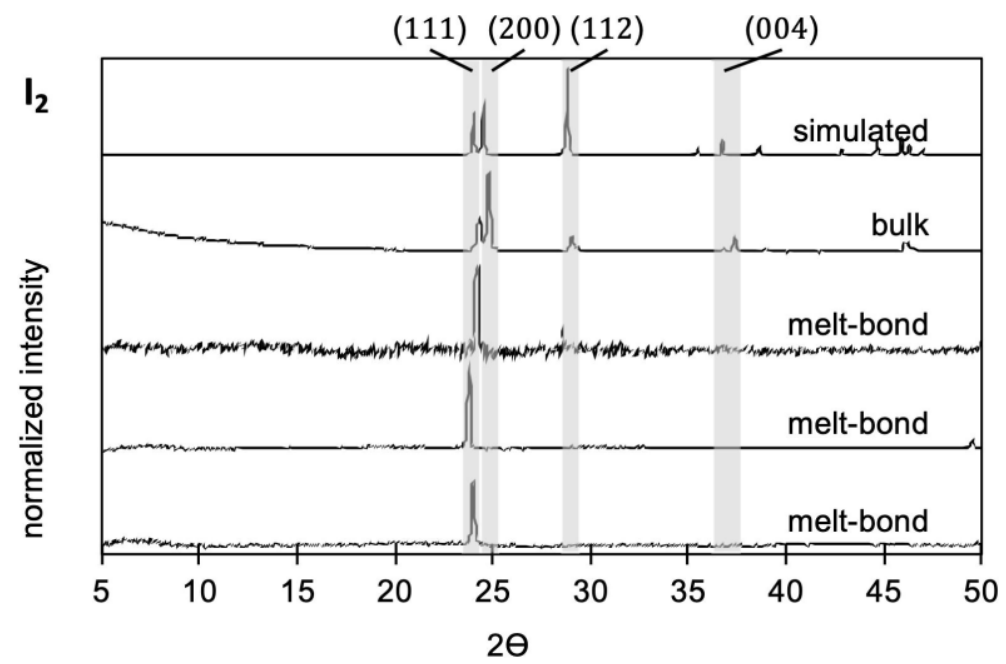

Figure S38. X-Ray Diffraction spectra for iodine. X-Ray Diffraction (XRD) spectra were generated from a Crystallographic Information File (CIF), ground powder (bulk), and three individual melt-bonded samples. 


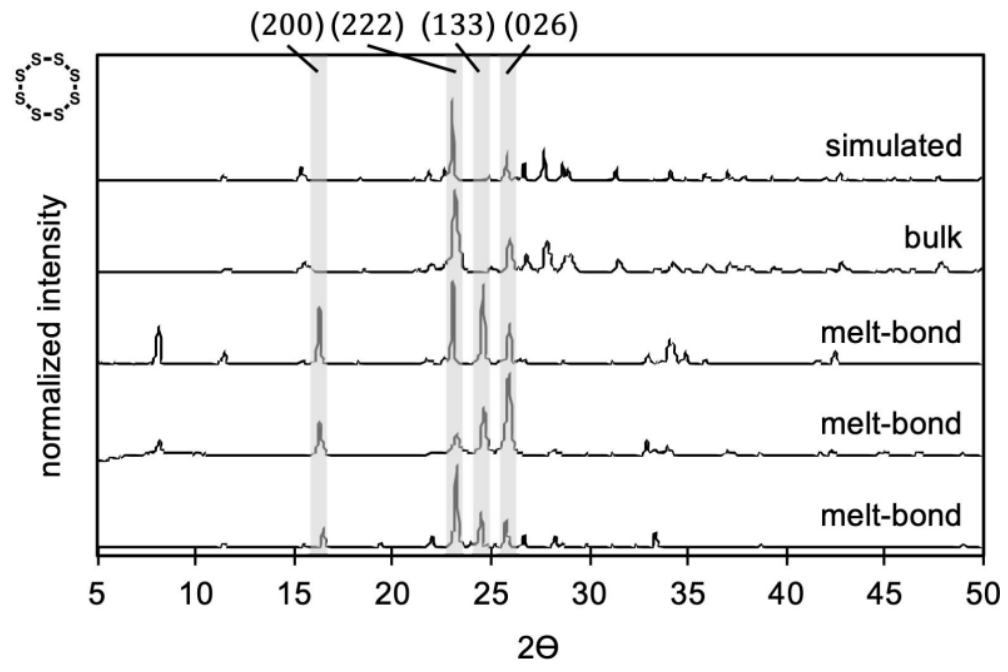

Figure S39. X-Ray Diffraction spectra for octacyclic sulfur. X-Ray Diffraction (XRD) spectra were generated from a Crystallographic Information File (CIF), ground powder (bulk), and three individual melt-bonded samples. 


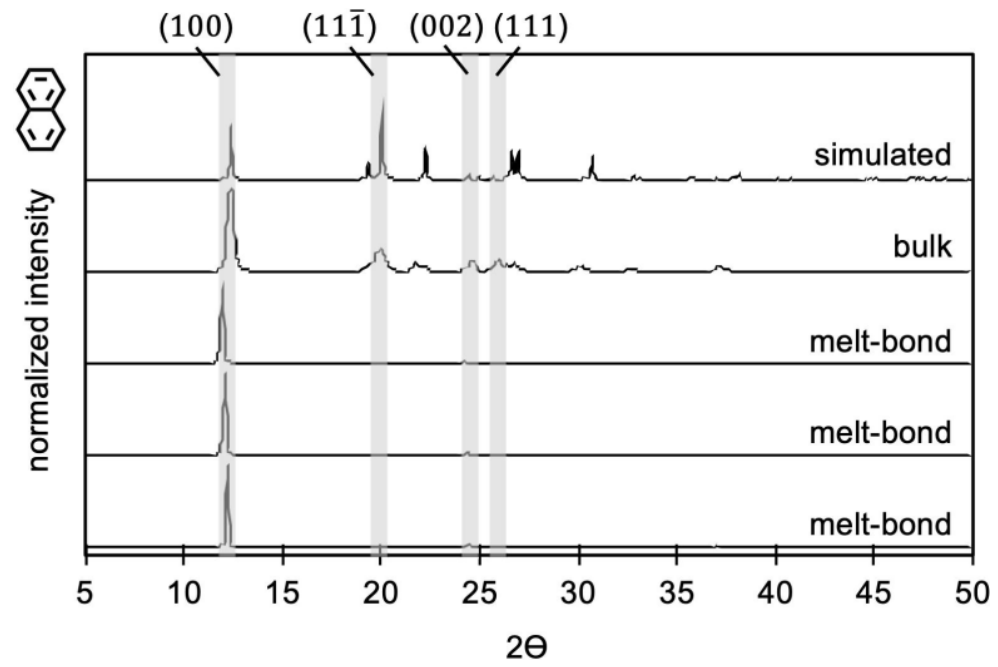

Figure S40. X-Ray Diffraction spectra for naphthalene. X-Ray Diffraction (XRD) spectra were generated from a Crystallographic Information File (CIF), ground powder (bulk), and three individual melt-bonded samples. 


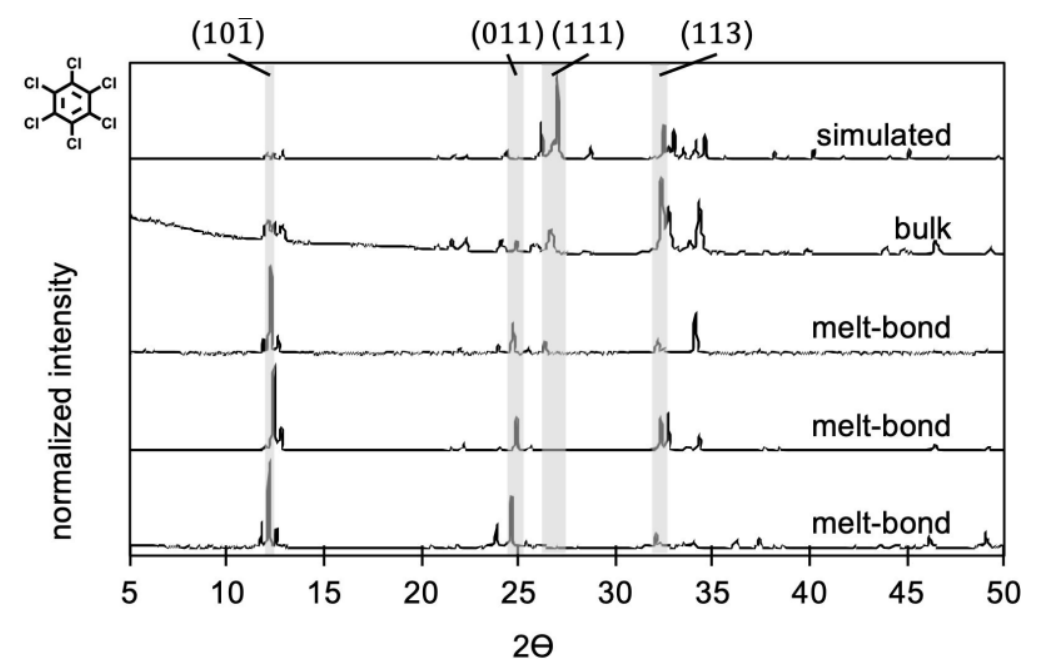

Figure S41. X-Ray Diffraction spectra for hexachlorobenzene. X-Ray Diffraction (XRD) spectra were generated from a Crystallographic Information File (CIF), ground powder (bulk), and individual three melt-bonded samples. 


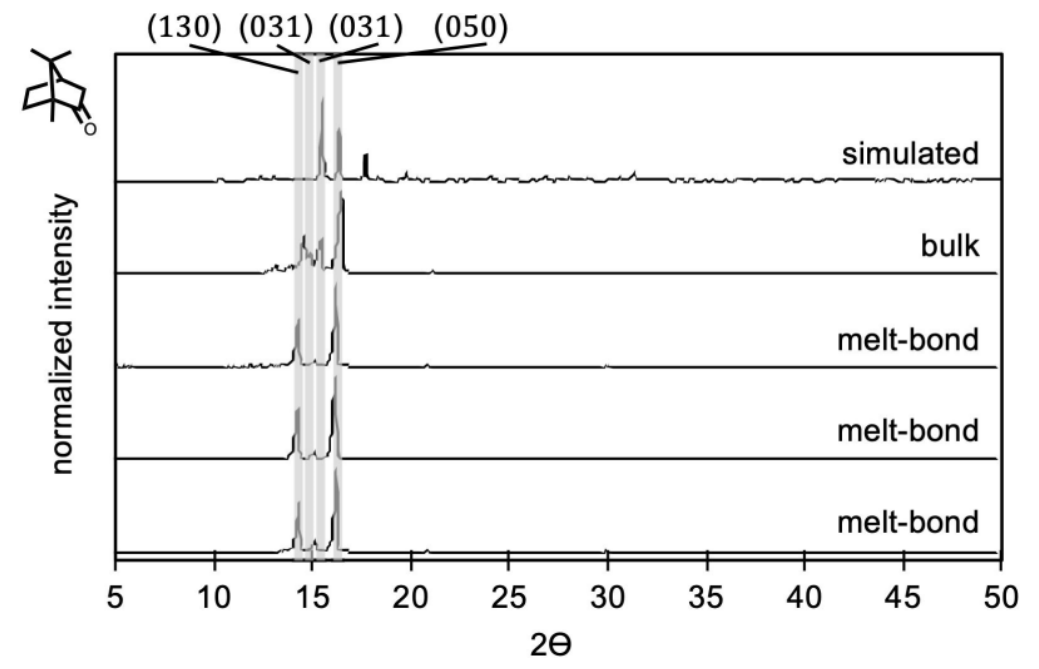

Figure S42. X-Ray Diffraction spectra for (+)-camphor. X-Ray Diffraction (XRD) spectra were generated from a Crystallographic Information File (CIF), ground powder (bulk), and three individual melt-bonded samples. 


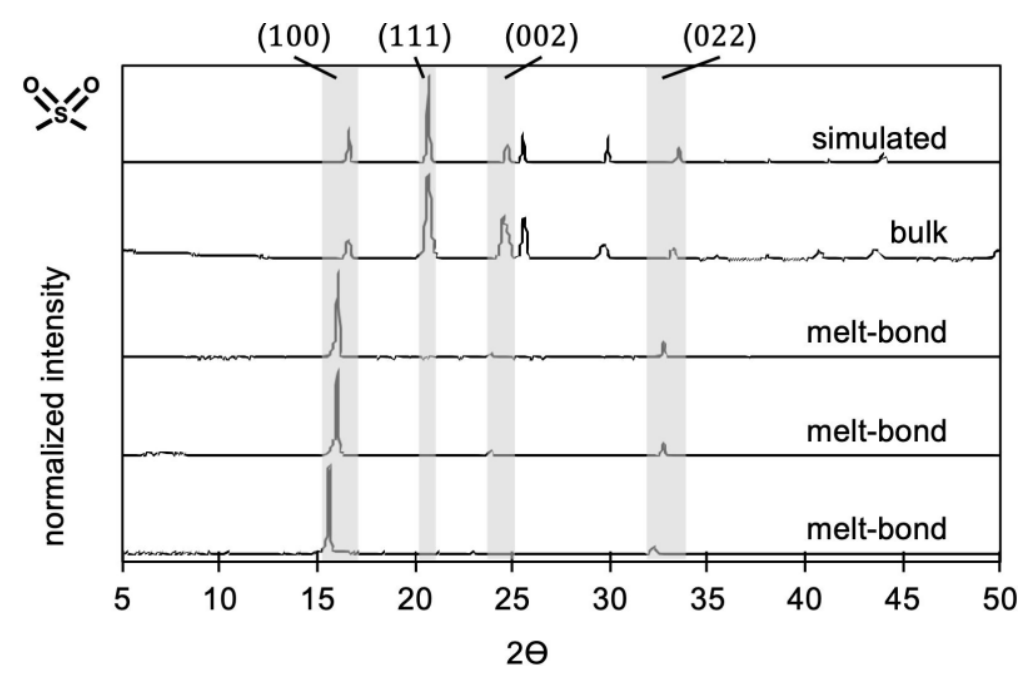

Figure S43. X-Ray Diffraction spectra for dimethyl sulfone. X-Ray Diffraction (XRD) spectra were generated from a Crystallographic Information File (CIF), ground powder (bulk), and individual three melt-bonded samples. 


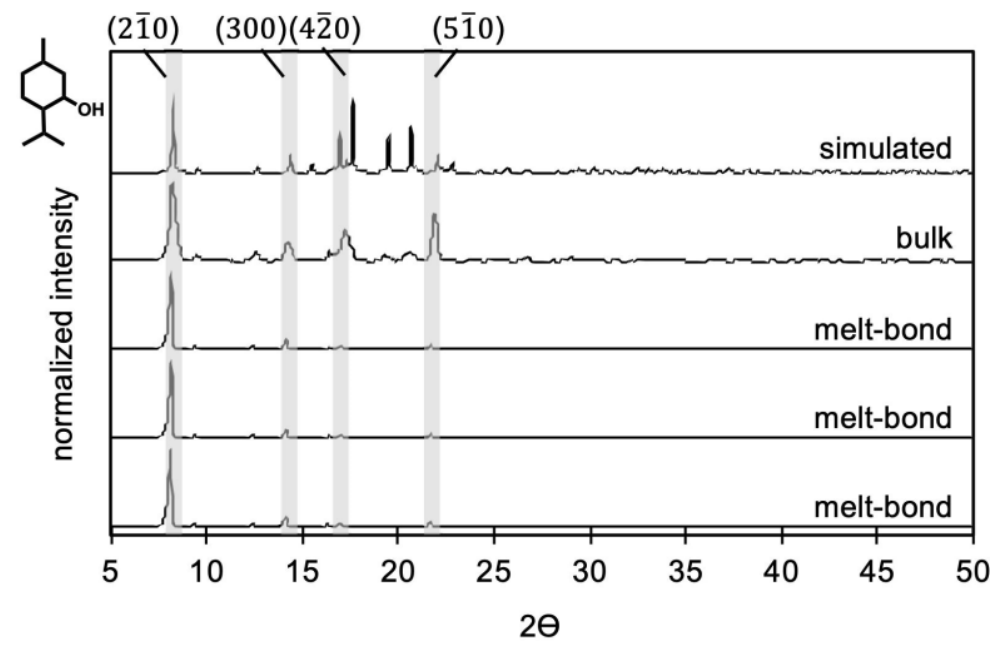

Figure S44. X-Ray Diffraction spectra for menthol. X-Ray Diffraction (XRD) spectra were generated from a Crystallographic Information File (CIF), ground powder (bulk), and three individual melt-bonded samples. 


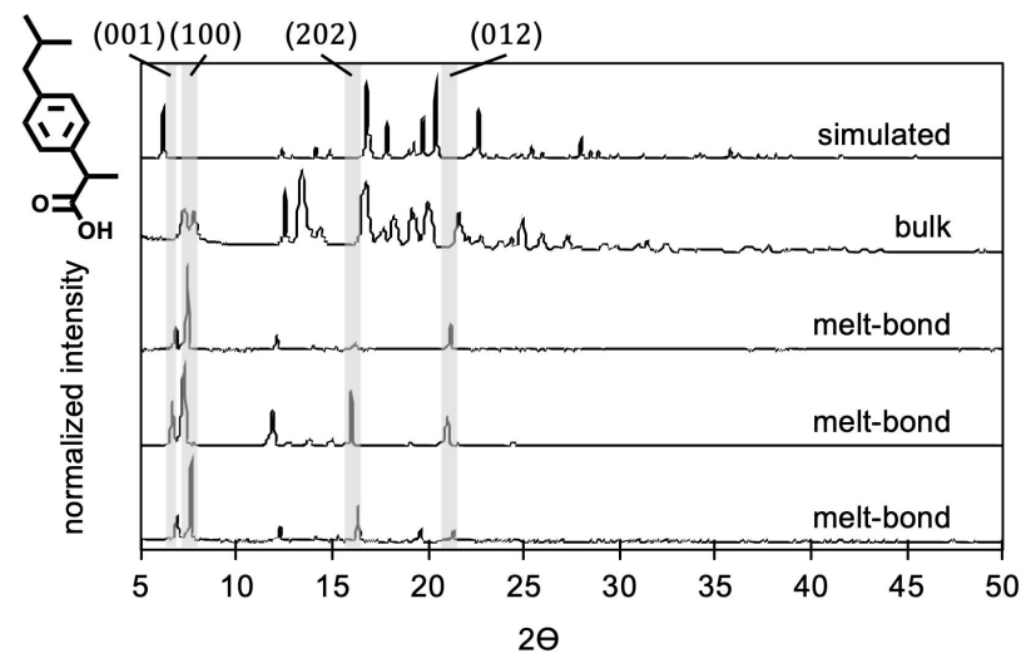

Figure S45. X-Ray Diffraction spectra for ibuprofen. X-Ray Diffraction (XRD) spectra were generated from a Crystallographic Information File (CIF), ground powder (bulk), and three individual melt-bonded samples. 


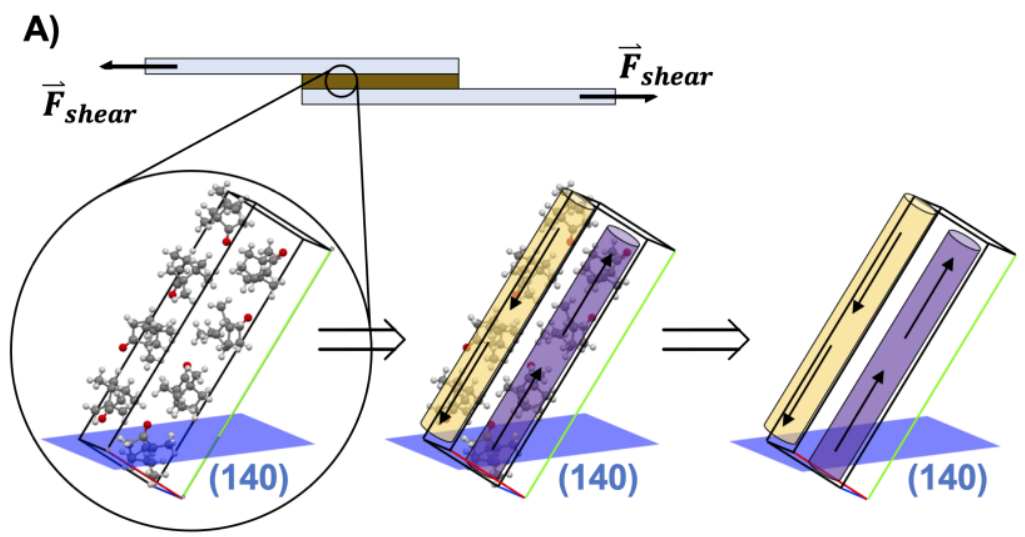

B)
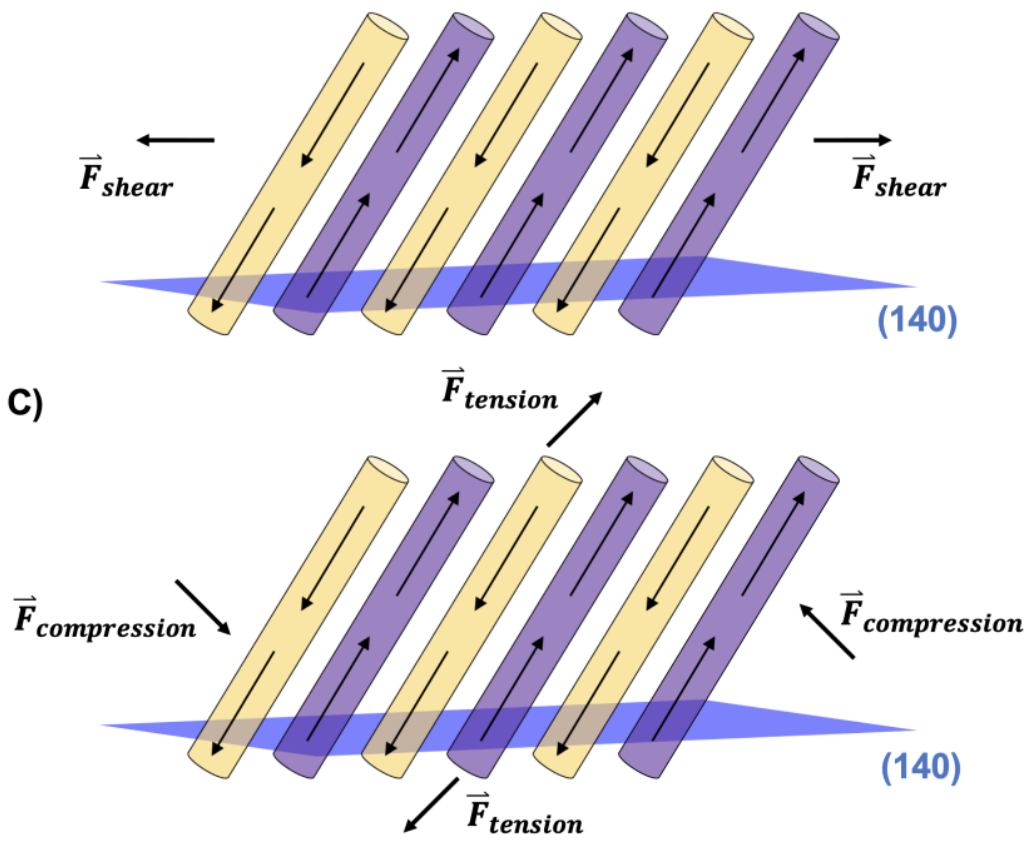

Figure S46. Visualizing shear and tension/compression stress states on the crystal structure of camphor in the (140) preferential orientation. (A) During melt-bonding of lap-shear samples on unmodified glass substrates, crystals of camphor are preferentially oriented such that the (140) crystal plane is parallel to the surface of the glass. In this orientation, the helices of camphor are angled at $37^{\circ}$ relative to the glass surface. These helices can be thought of as columns of strong dipole-dipole intermolecular interactions (the direction of these interactions is illustrated by arrows). (B) When a sample is subjected to lap-shear testing, a shear force is applied to the sample and increased at a rate of $50 \mathrm{~N} \mathrm{~min}^{-1}$ until sample failure. (C) By changing the coordinate system (an equivalent procedure to switching between cartesian and polar coordinates), the applied stress state can alternatively be viewed in terms of tension and compression. When doing so, it is evident that the strong intermolecular interactions in the camphor helices are well-aligned with the applied tensile forces. We hypothesize that this alignment is a major contribution to the mechanical and adhesive performance of camphor. 


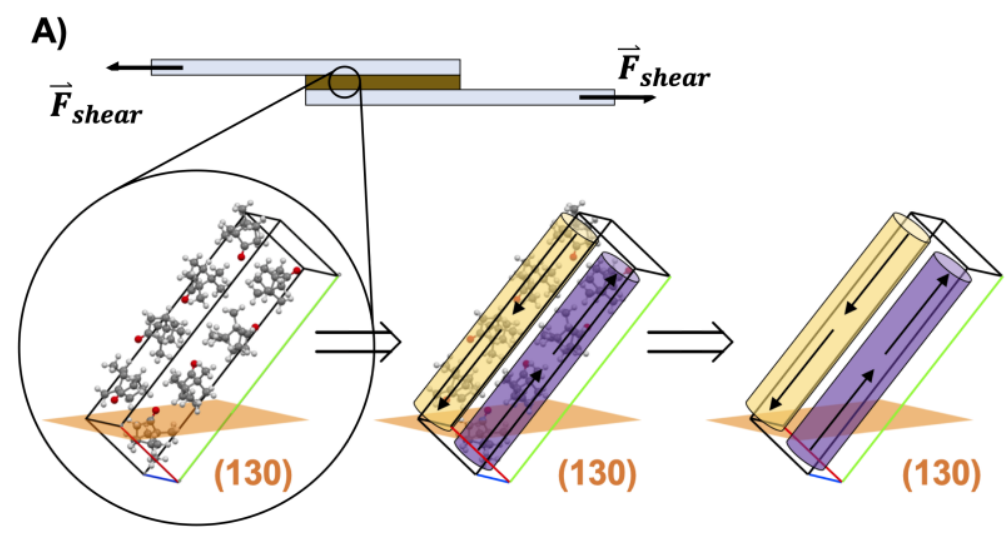

B)
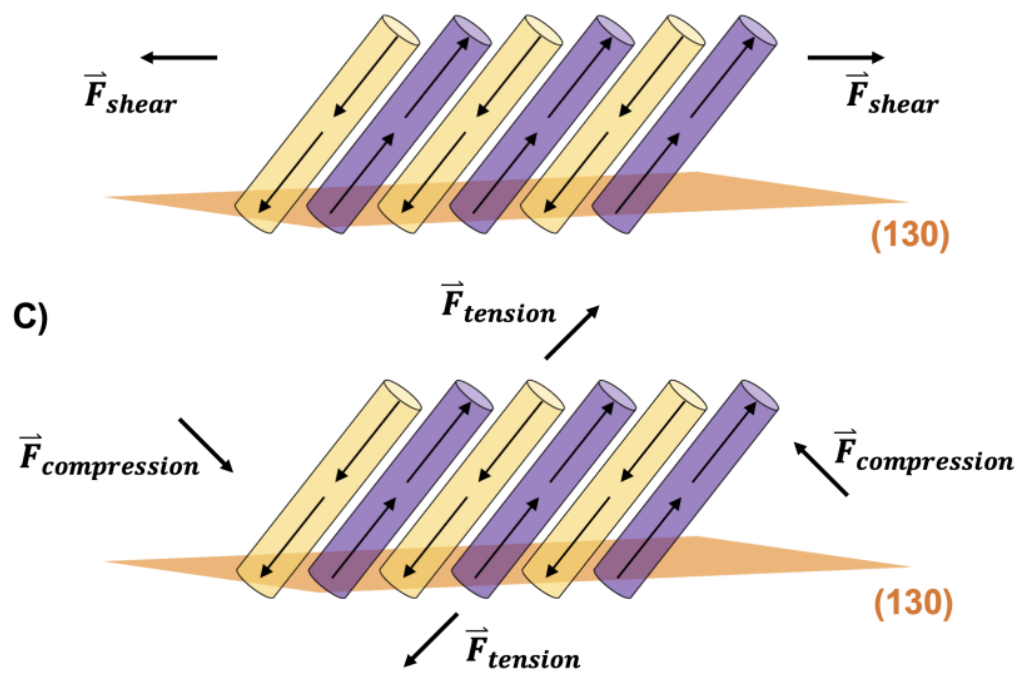

Figure S47. Visualizing shear and tension/compression stress states on the crystal structure of camphor in the (130) preferential orientation. (A) During melt-bonding of lap-shear samples on unmodified glass substrates, crystals of camphor are preferentially oriented such that the (130) crystal plane is parallel to the surface of the glass. In this orientation, the helices of camphor are angled at $45^{\circ}$ relative to the glass surface. These helices can be thought of as columns of strong dipole-dipole intermolecular interactions (the direction of these interactions is illustrated by arrows). (B) When a sample is subjected to lap-shear testing, a shear force is applied to the sample and increased at a rate of $50 \mathrm{~N} \mathrm{~min}^{-1}$ until sample failure. (C) By changing the coordinate system (an equivalent procedure to switching between cartesian and polar coordinates), the applied stress state can alternatively be viewed in terms of tension and compression. When doing so, it is evident that the strong intermolecular interactions in the camphor helices are well-aligned with the applied tensile forces. We hypothesize that this alignment is a major contribution to the mechanical and adhesive performance of camphor. 

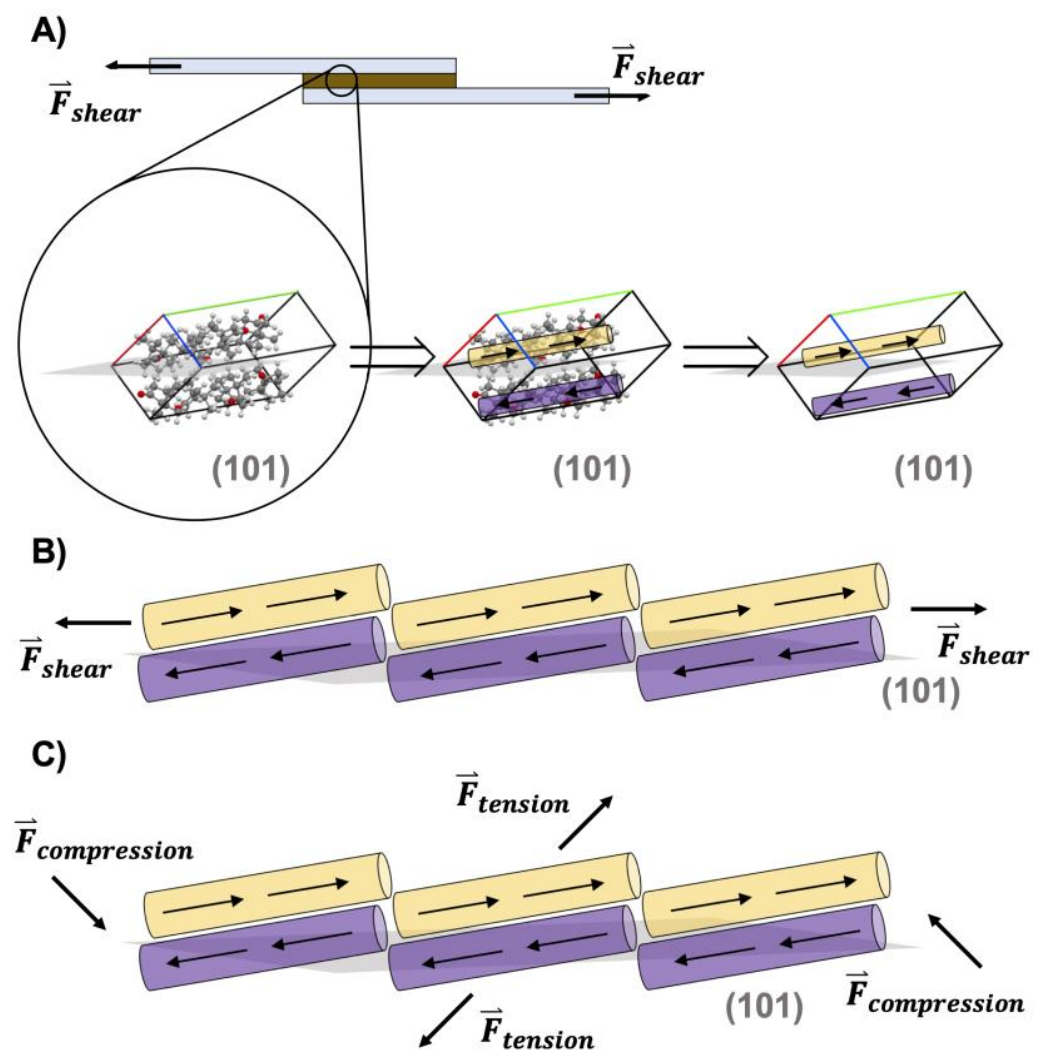

Figure S48. Visualizing shear and tension/compression stress states on the crystal structure of camphor in the (101) preferential orientation. (A) During melt-bonding of lap-shear samples on trichloro(octyl)silane-modified glass substrates, crystals of camphor are preferentially oriented such that the (101) crystal plane is parallel to the surface of the glass. In this orientation, the helices of camphor are angled nearly parallel to the glass surface. These helices can be thought of as columns of strong dipole-dipole intermolecular interactions (the direction of these interactions is illustrated by arrows). (B) When a sample is subjected to lap-shear testing, a shear force is applied to the sample and increased at a rate of $50 \mathrm{~N} \mathrm{~min}^{-1}$ until sample failure. (C) By changing the coordinate system (an equivalent procedure to switching between cartesian and polar coordinates), the applied stress state can alternatively be viewed in terms of tension and compression. When doing so, it is evident that the strong intermolecular interactions in the camphor helices are poorly aligned with the applied tensile forces. We hypothesize that this alignment is a major contribution to the lack of mechanical and adhesive performance of camphor on trichloro(octly)silane-modified substrates. 


\section{SXI. Quantifying the Mechanism of Failure for Melt-Bonded Samples}

Optical micrographs of each sample were obtained as discussed above (Section SVI). The surface area of the polycrystalline film remaining on both the top (Atop) and bottom (A $A_{\text {bottom }}$ ) substrates were quantified using the same procedure as for the adhesive surface area $\left(A_{\text {adhesive }}\right)$. From these measurements, percent cohesive failure and percent adhesive failure were calculated with Equations S5-S6, respectively. Cohesive failure is assumed to be $0 \%$ in the case that Equation $\mathbf{S} 5$ produces a negative value. All data is presented in Table S4.

$$
\begin{gathered}
\text { cohesive failure }(\%)=\frac{A_{\text {top }}+A_{\text {bottom }}}{A_{\text {adhesive }}}-1 \\
\text { adhesive failure }(\%)=1-\text { cohesive failure }(\%)
\end{gathered}
$$

Table S4. Percent adhesive vs cohesive failure of molecular solid adhesives.

\begin{tabular}{ccc}
\hline Molecular solid & Percent Adhesive Failure (\%) & Percent Cohesive Failure (\%) \\
\hline lodine & $87 \pm 34$ & $13 \pm 34$ \\
Octacyclic sulphur & $95 \pm 18$ & $5 \pm 18$ \\
Naphthalene & $95 \pm 10$ & $5 \pm 10$ \\
Hexachlorobenzene & $85 \pm 36$ & $15 \pm 36$ \\
Camphor & $24 \pm 6$ & $76 \pm 6$ \\
Dimethyl sulfone & $65 \pm 29$ & $35 \pm 29$ \\
(-)-Menthol & $95 \pm 26$ & $5 \pm 26$ \\
lbuprofen & $78 \pm 12$ & $22 \pm 12$ \\
\hline
\end{tabular}




\section{SXII. Generating Electron Surface Potential Plots}

The lowest energy structure of each adhesive was optimized to full convergence with density functional theory (DFT) at the B3LYP/6-311++(3d,2p) level of theory in a gas phase model using Gaussian'09. Electron density maps (at the same isosurface value) were visualized in GaussView5 and generated from electrostatic potentials computed through SCF electron density at the same level of theory utilized above.

\section{SXIII. Generating Hirshfeld Surfaces and Fingerprint Plots}

Crystallographic information files (CIFs) for each material studied were imported into Crystal Explorer software (Version: 17.5, Revision: f4e298a). ${ }^{531}$ The Surface Generation dialogue box was then used to calculate a Hirshfeld surface (Property: none, Resolution: high). Finally, the Display Fingerprint Plot option was utilized to calculate the fingerprint plot for each material. ${ }^{532}$ Standard software options were then used to save/export and analyze the plot.

\section{SXIV. Casting Pellets of Molecular Solids for Cohesive Measurements}

Solids were melted in a $100-\mathrm{mL}$ beaker over a heat gun, then cast a PTFE mold to produce cylindrical solid pellets for cohesion analysis. The mold was fabricated by drilling a 0.25 " diameter hole through a $1.5^{\prime \prime}$ long 2" diameter cylinder of PTFE with a drill press. Once the material had fully cooled and crystallized, the pellet was removed from the mold. On occasion, the blunt end of a $0.25^{\prime \prime}$ diameter drill bit was used to press the pellet 
from the mold. The ends of the pellet were trimmed to produce right angles at the ends of the cylinder once removed from the mold.

\section{SXV. Shear Testing of Pellets for Cohesive Measurements}

We employed a cohesive shearing protocol similar to ASTM D732 to quantify the maximum force required to shear a molecular solid pellet. ${ }^{\mathrm{S} 33}$ Assemblies were placed into a custom-machined two-piece cohesive testing rig (Fig. S49-S51), which was attached to the shear testing machine (Instron 5544). Samples were sheared at a loading rate of $30 \mathrm{~N} \mathrm{~min}-1$ until failure. The force and extension of the shear testing was recorded using Blue Hill software, exported as comma-separated value files, and analyzed in Microsoft Excel. The maximum force is the highest force withstood by the pellet before failing. The maximum extension is the extension value at the maximum force. For analogy with adhesive lap-shear testing, we introduced the parameter $\tau(\mathrm{kPa})$ as the ratio of the maximum force $(\mathrm{N})$ withstood before failure, to the cross-sectional area $\left(\mathrm{cm}^{2}\right)$ of the pellet

(Eq. S2). As pellets were cast from a mold of known geometry, the cross-sectional area was $A=0.316 \mathrm{~cm}^{2}$. Likewise, we introduced the parameter $\gamma$ as the ratio of the extension of the cohesion testing rig $(\mathrm{mm})$, to the effective thickness of the sample $(\mathrm{mm})$ (Eq. 3). The effective thickness of the sample was the length of the pellet that spanned the small gap between the two pieces of the cohesive testing rig, which was equal to $t=1.5 \mathrm{~mm}$. In some cases, pronounced crumbling of the polycrystalline sample was evident during mechanical testing. These cases were evident by the jagged stress-strain profiles of the 
samples (Fig. S52). However, they were analyzed with the same procedure as all other samples. 

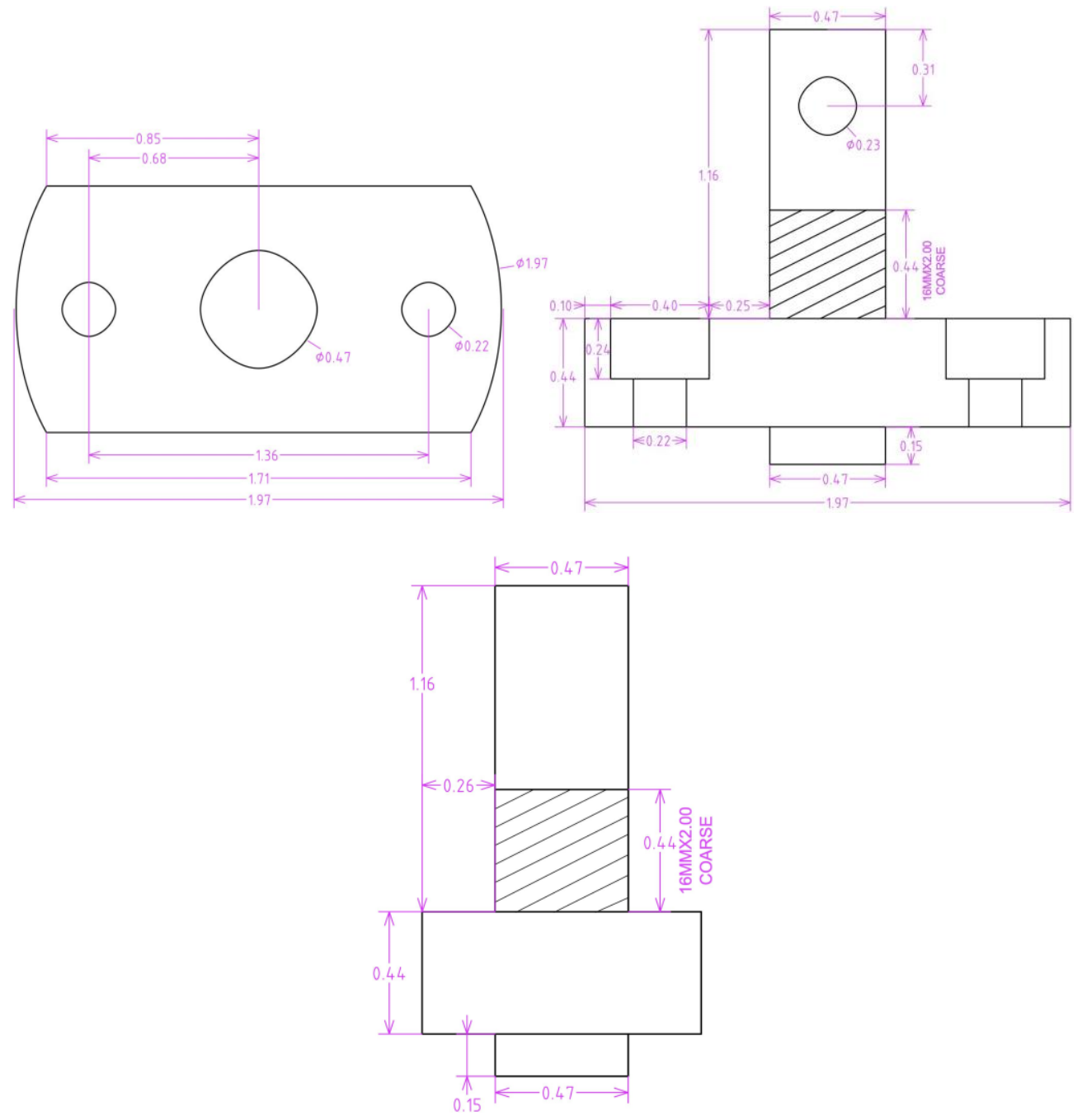

Figure S49. Technical drawing showing the top piece of the cohesive testing fixture. 


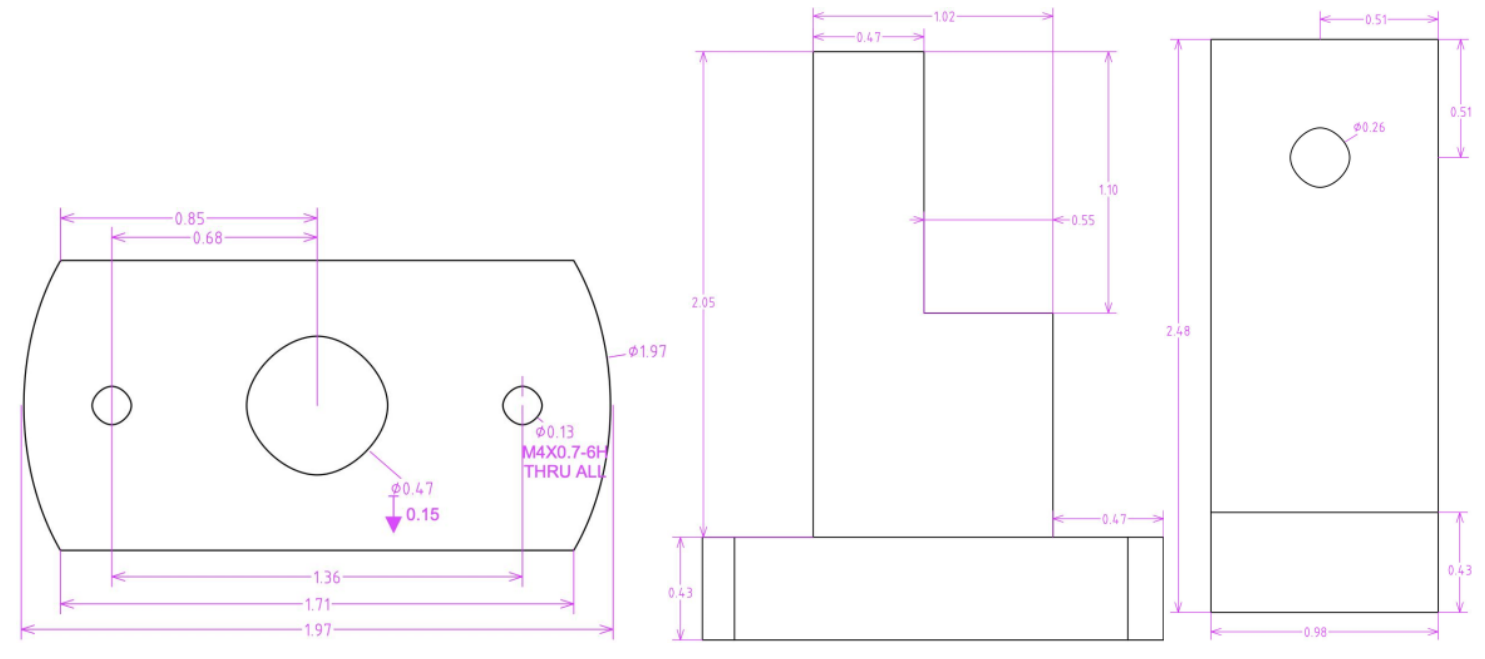

Figure S50. Technical drawing showing the bottom piece of the cohesive testing fixture. 


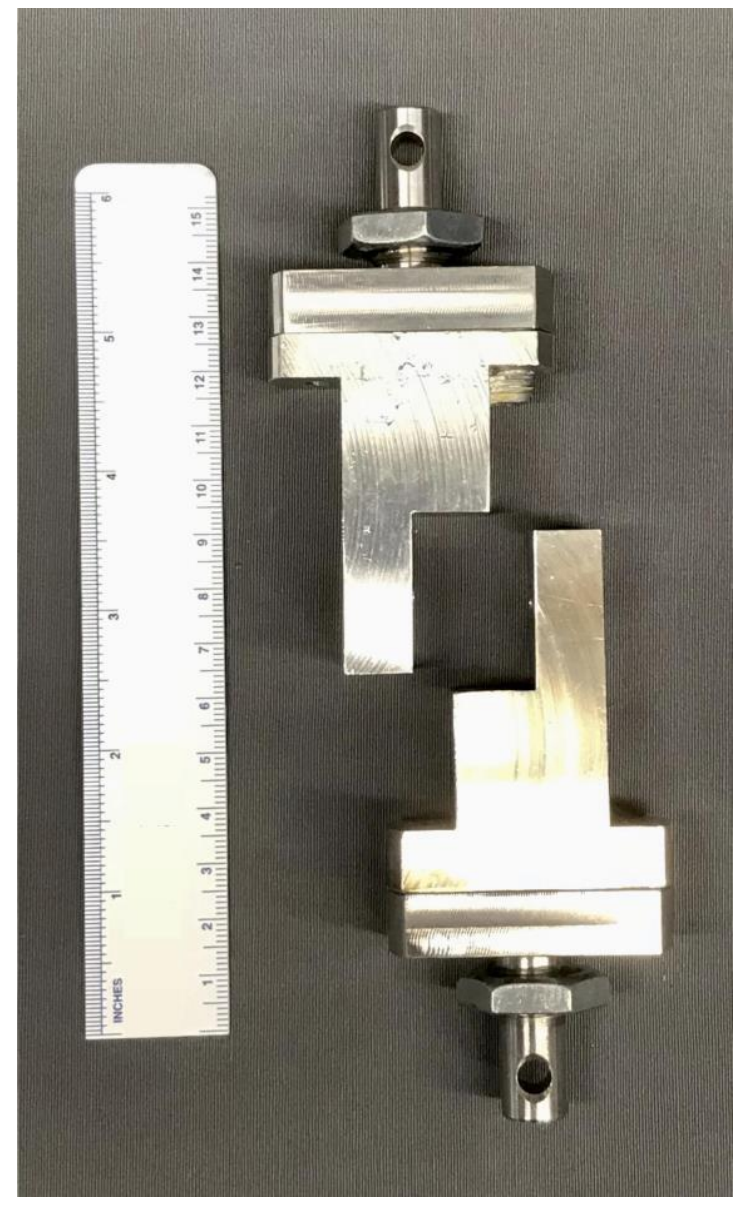

Figure S51. Optical image of the cohesive testing fixture. 


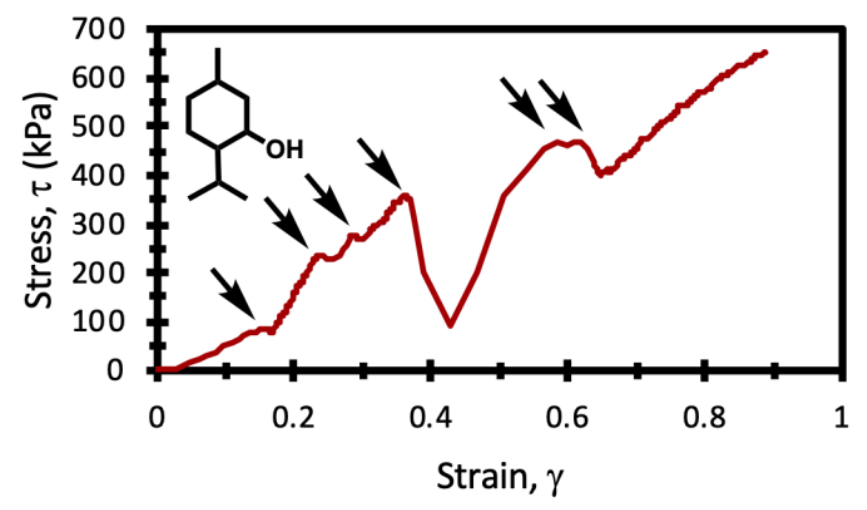

Figure S52. Pronounced crumbling of the polycrystalline samples was evident for some cohesive samples. Crumbling was evident in sudden decreases in the applied stress during testing. These events are marked by the black arrows in the representative stress-strain plot for a sample of (-)-menthol above. All samples, were analyzed using the sample procedure, discussed above, regardless of the degree of crumbling evident. 


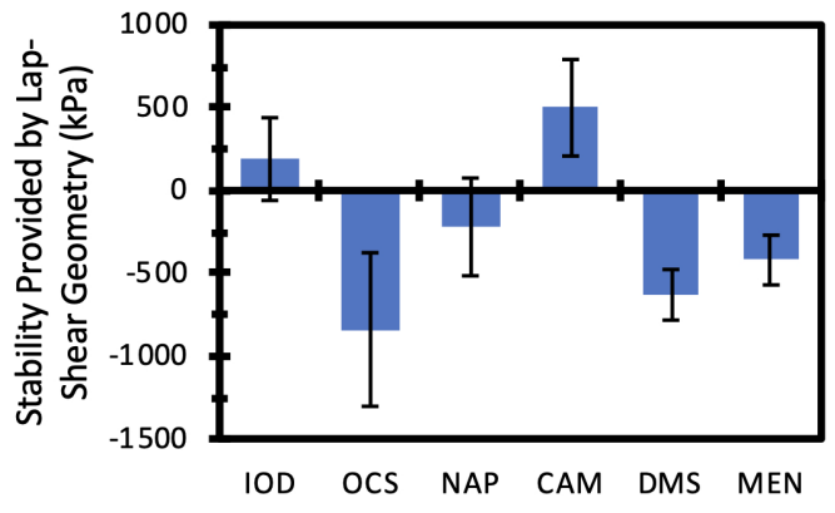

Figure S53. Relative stability of lap-shear relative to bulk-shear samples. Camphor is stabilized by $\tau=500 \pm 292 \mathrm{kPa}$ in the lap-shear, relative to the bulk shear geometry. Conversely, octacyclic sulfur, dimethyl sulfone and menthol are stabilized by $\tau=840 \pm 464,630 \pm 153$ and $420 \pm 150 \mathrm{kPa}$ respectively. There is no statistical difference in the stress at fracture between lap-shear and bulk-shear samples for iodine and naphthalene. Hexachlorobenzene and ibuprofen could not be analyzed in bulk shear. 


\section{SXVI.Melt-Bonding Substrates with Molecular Solids for Release of}

\section{Adherends by Sublimation}

The melt-bonding procedure for release by sublimation experiments was analogous to that used for shear testing experiments with a few notable changes. First, the substrates were bonded without the aid of the aluminum bonding frame. Second, PTFE washers were not used to control the thickness of the adhesive layer. Finally, a small piece of glass $\left(0.75 \times 1 \mathrm{in}^{2}\right)$ and an aluminum grate $(0.25$ " diameter holes arranged in a hexagonal pattern with $0.375^{\prime \prime}$ in between each hole) were used as adherends. The grate was selected to facilitate mass transfer through its macroscopic pores during sublimation, and a smaller piece of glass was utilized to enhance the visual impact of release.

During bonding, substrates were preheated at the melt-bonding temperature before placing the molecular solid between the adherends. Once the hotplate signaled it had reached the set temperature, the molecular solid was placed on the bottom (glass) substrate. The mass of molecular solid deposited was selected to result in $\sim 40 \mathrm{mg}$ of adherend material. The top (aluminum) substrate was then placed on the bottom substrate. A mass of $112.3 \mathrm{~g}$ was placed on top of the top substrate to flatten the assembly and spread the melted molecular solid. The fixture was then transferred from the hotplate to a lab jack (ChemGlass, CG-3054). The lab jack acted as a cooling stage for the assembly of substrates and melted molecular solid. As the molecular solid cooled it fused and bonded the substrates. 


\section{SXVII. Release of Adherends by Sublimation}

Adhered assemblies were prepared for release by sublimation experiments as described in Section SXVI. Bonded assemblies were loaded into a sublimation chamber (Kimble 746125-0001), heated to $95 \%$ of the melting point of the material, and placed under a high-vacuum of 50-100 mTorr (Fig. S54, Movie S1-S2). Progress of the sublimation was monitored, and, in some cases, could be qualitatively judged by the visual accumulation of adhesive material on the inside of the sublimation chamber and/or cold trap. Release of adherends was judged to be complete when the glass substrate fell off the aluminum grate under the force of gravity. Sublimation rates were calculated by dividing the mass of adhesive lost throughout the debonding process, by the time to release. Data are presented in Table S5. 


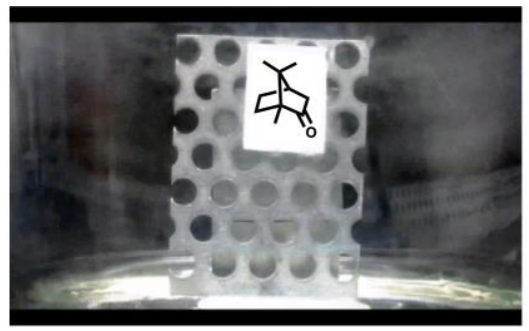

$63^{\circ} \mathrm{C}, 75$ mTorr $30 \mathrm{~min}$

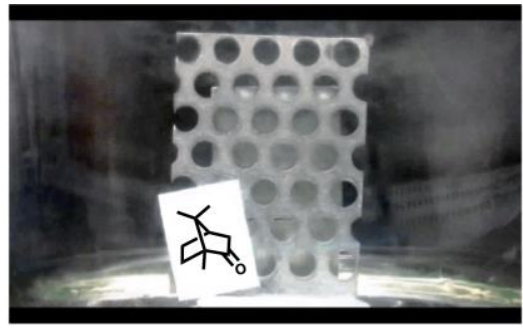

Figure S54. Release-by-sublimation of glass and porous aluminum adherends. A small glass adherend was melt-bonded to a porous aluminum substrate with naphthalene. The sample was place into a sublimation chamber, heated to $63^{\circ} \mathrm{C}$, and subjected to a vacuum of 75 mTorr. After $30 \mathrm{~min}$, the naphthalene adhesive had sublimed, resulting in the release of the adherends under the force of gravity. 
Table S5. On-demand release of adherends by sublimation. ${ }^{a}$

\begin{tabular}{cccc}
\hline Molecular solid & Temperature $\left({ }^{\circ} \mathrm{C}\right)$ & Time to Release $(\mathrm{min})$ & $\begin{array}{c}\text { Sublimation Rate } \\
\left(\mathrm{mg} \mathrm{min}^{-1}\right)\end{array}$ \\
\hline Iodine & 108 & 25 & 3.22 \\
Octacyclic sulphur & 109 & $--^{\mathrm{b}}$ & $--^{\mathrm{b}}$ \\
Naphthalene & 76 & 30 & 1.55 \\
Hexachlorobenzene & 219 & $--^{\mathrm{c}}$ & $--^{\mathrm{c}}$ \\
Camphor & 166 & 6 & 1.72 \\
Dimethyl sulfone & 104 & 90 & 0.46 \\
(-)-Menthol & 42 & 105 & 0.44 \\
Ibuprofen & 72 & $--^{\mathrm{b}}$ & $--^{\mathrm{b}}$ \\
\hline
\end{tabular}

a Sublimation experiments were performed at 50-100 mTorr.

b Substrates did not release within $24 \mathrm{~h}$.

${ }^{\mathrm{b}}$ Adhesive was too volatile to melt-bond to a porous substrate.

\section{SXVIII. Thermal Analysis of Molecular Solids}

Each molecular solid was analyzed via differential scanning calorimetry (DSC) to confirm that only one polymorph was present. A DSC Q2000 (TA Instruments) was used to cycle a $3 \mathrm{mg}$ sample of each solid across a broad temperature range roughly centering on the literature melting point. Each sample was placed through a heat/cool cycle prior to data collection to erase any previous thermal history and to mimic the effects of the melt-bonding and casting processes. The presence of only one polymorph was confirmed by a single melting peak present in the thermogram of each sample. 
All thermogravimetric experiments were carried out utilizing a TGA 55 (TA Instruments). Data was analyzed in TA7000 Thermal Analysis Software (Hitachi, Version 11.1). Two experiments were completed on each molecular solid. First, a sample of each solid was heated from room temperature to $350{ }^{\circ} \mathrm{C}$ at a constant rate of $10{ }^{\circ} \mathrm{C} \min ^{-1}$. Second, sample of each solid was heated to $95 \%$ of the decomposition temperature of the material at a constant rate of $20^{\circ} \mathrm{C} \mathrm{min}^{-1}$, then held at constant temperature for $3 \mathrm{~h}$. The sublimation rate of each solid was quantified by finding the slope of a line of best fit for the plot of sample mass as a function of temperature. All data collected are presented in Figures S55-S62. 

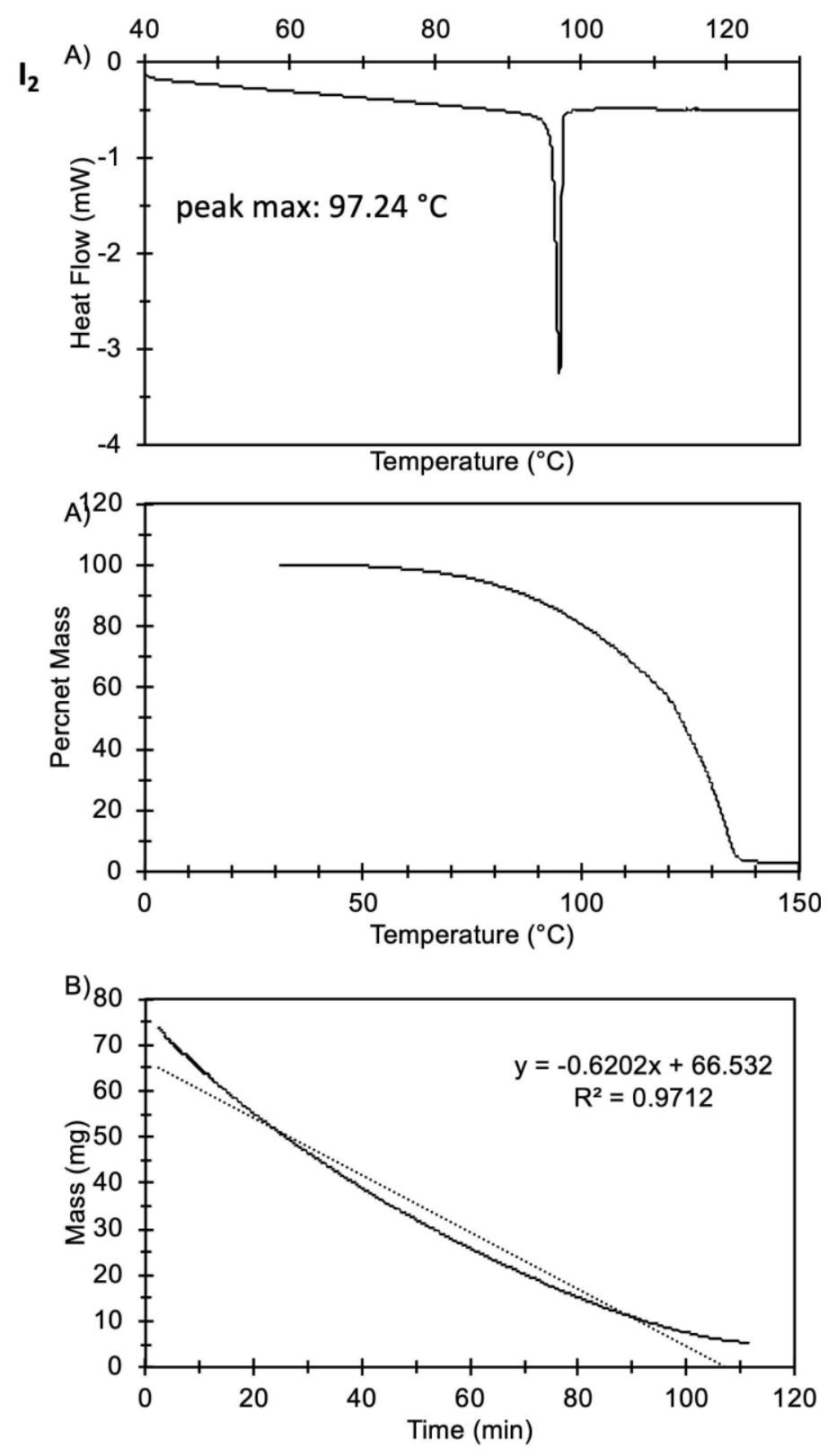

Figure S55. Thermal analysis of iodine. A) Differential scanning calorimetry. B) Temperature ramped from room temperature to $350{ }^{\circ} \mathrm{C}$ at $10^{\circ} \mathrm{C} \mathrm{min}^{-1}$. C) Temperature held constant at $70{ }^{\circ} \mathrm{C}$. 

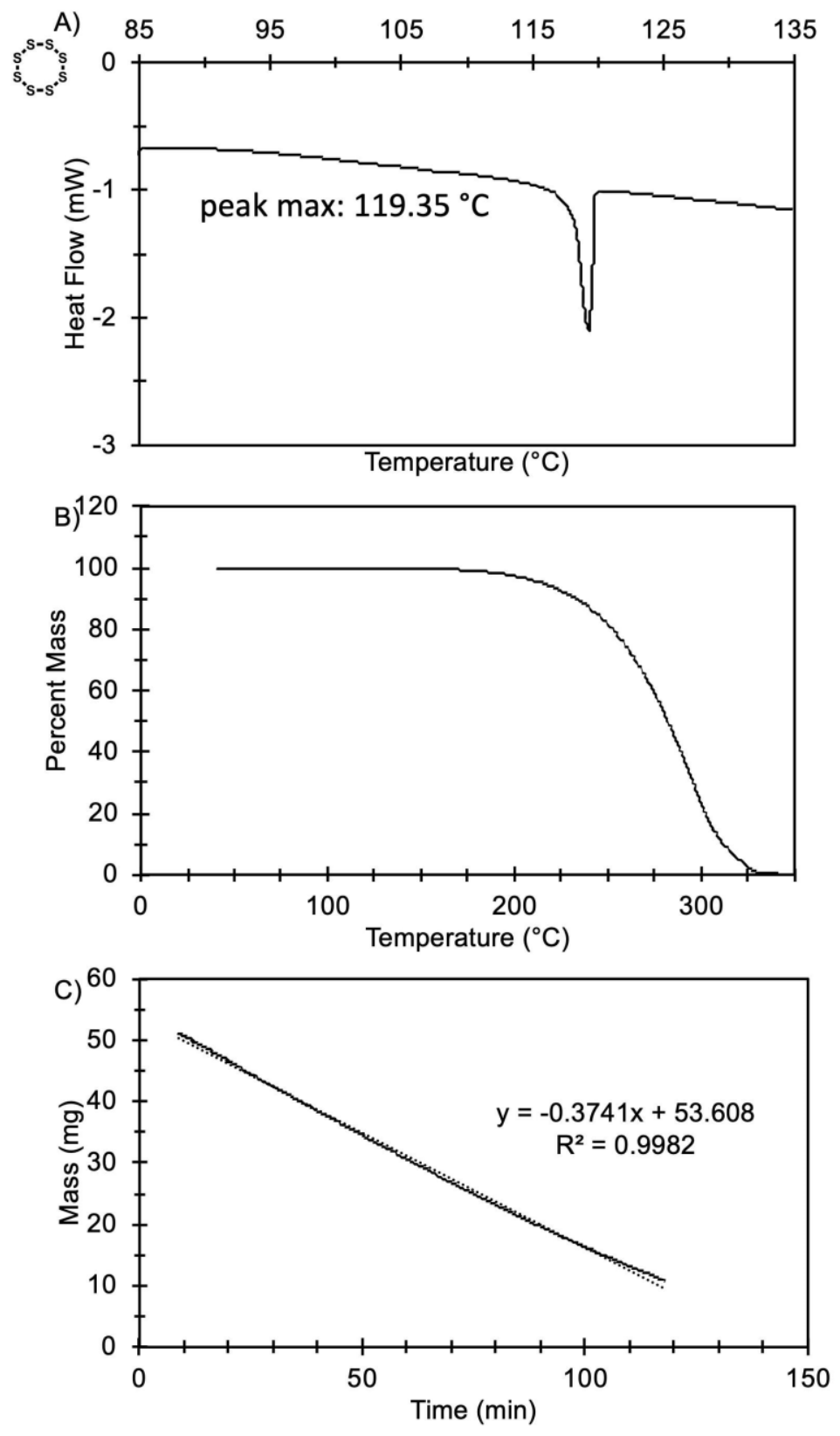

Figure S56. Thermal analysis of octacyclic sulfur. A) Differential scanning calorimetry.

B) Temperature ramped from room temperature to $350{ }^{\circ} \mathrm{C}$ at $10{ }^{\circ} \mathrm{C} \mathrm{min}^{-1}$. C) Temperature held constant at $198^{\circ} \mathrm{C}$. 

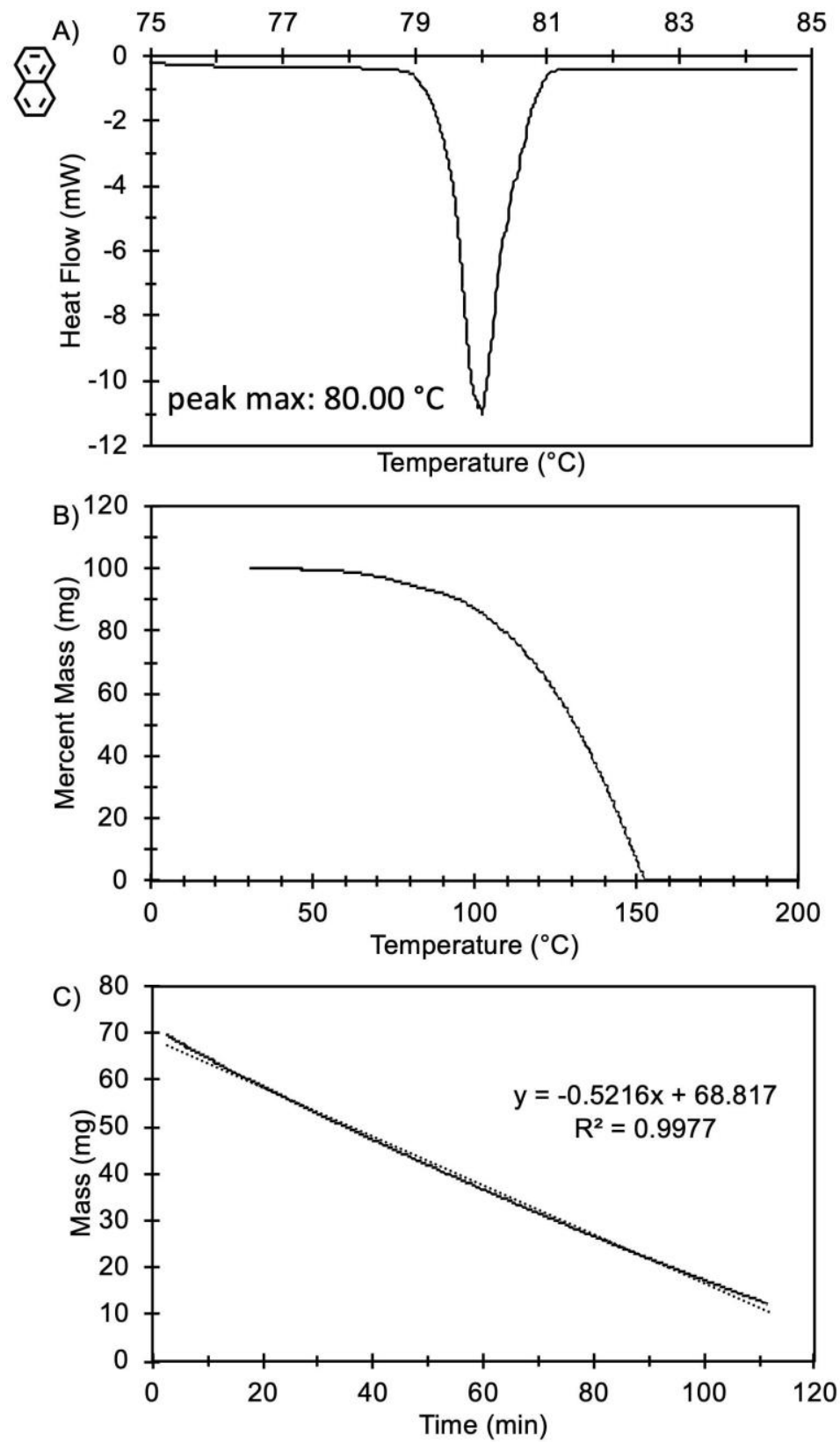

Figure S57. Thermal analysis of naphthalene. A) Differential scanning calorimetry. B) Temperature ramped from room temperature to $350{ }^{\circ} \mathrm{C}$ at $10^{\circ} \mathrm{C} \mathrm{min}^{-1}$. C) Temperature held constant at $76{ }^{\circ} \mathrm{C}$. 

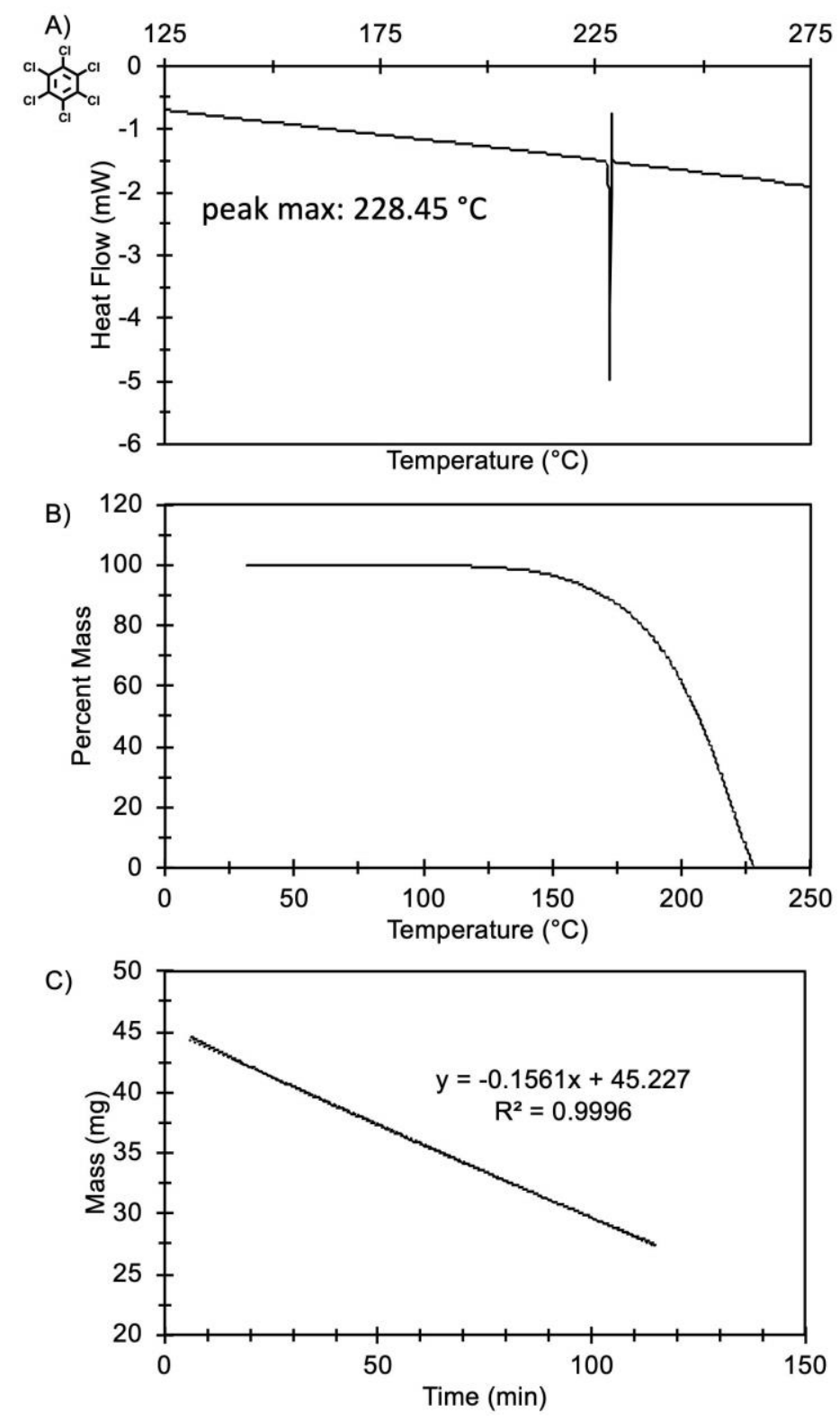

Figure S58. Thermal analysis of hexachlorobenzene. A) Differential scanning calorimetry. B) Temperature ramped from room temperature to $350{ }^{\circ} \mathrm{C}$ at $10^{\circ} \mathrm{C} \mathrm{min}^{-1}$. C) Temperature held constant at $141^{\circ} \mathrm{C}$. 

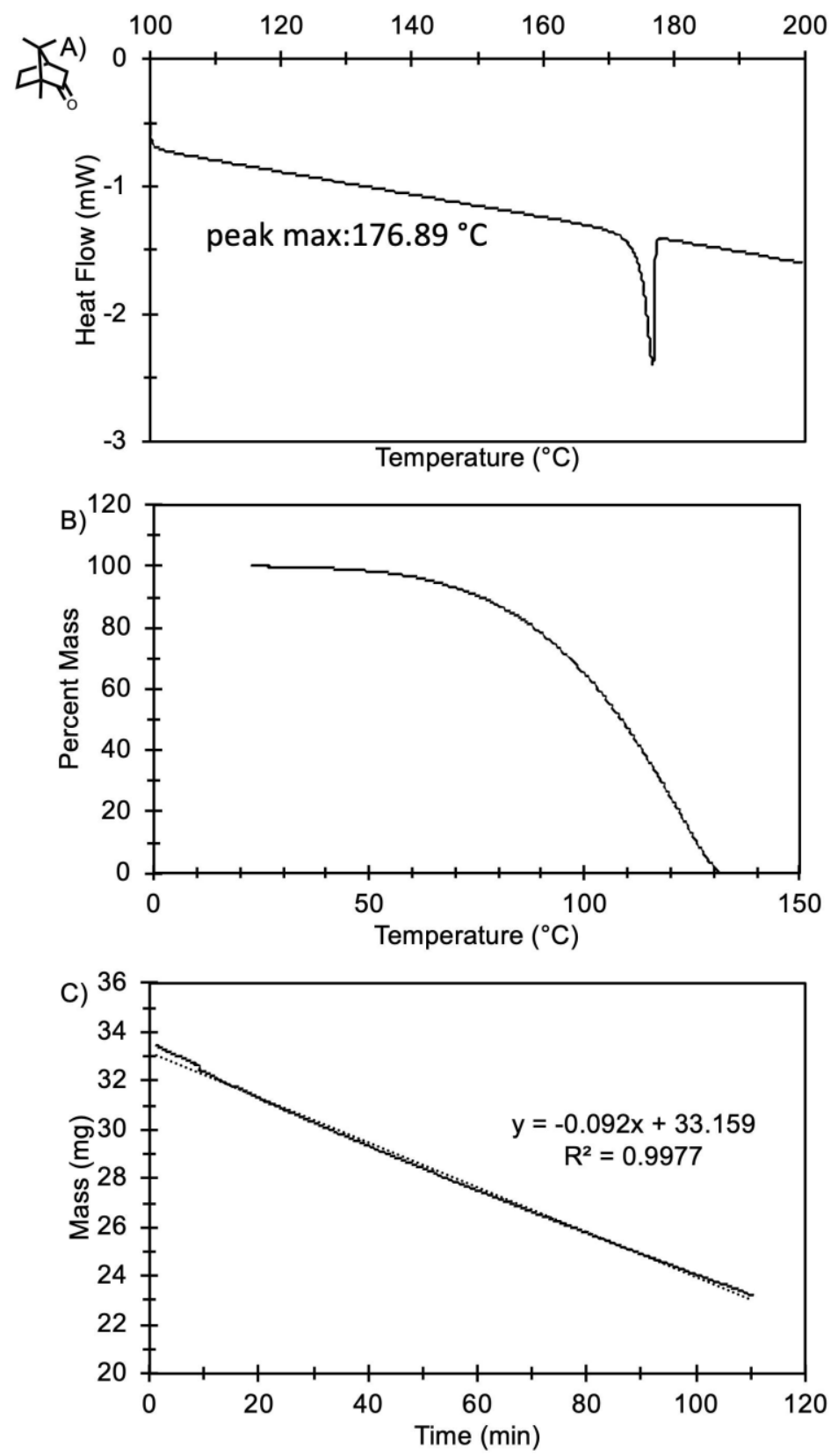

Figure S59. Thermal analysis of camphor. A) Differential scanning calorimetry. B) Temperature ramped from room temperature to $350{ }^{\circ} \mathrm{C}$ at $10^{\circ} \mathrm{C} \mathrm{min}^{-1}$. C) Temperature held constant at $48^{\circ} \mathrm{C}$. 

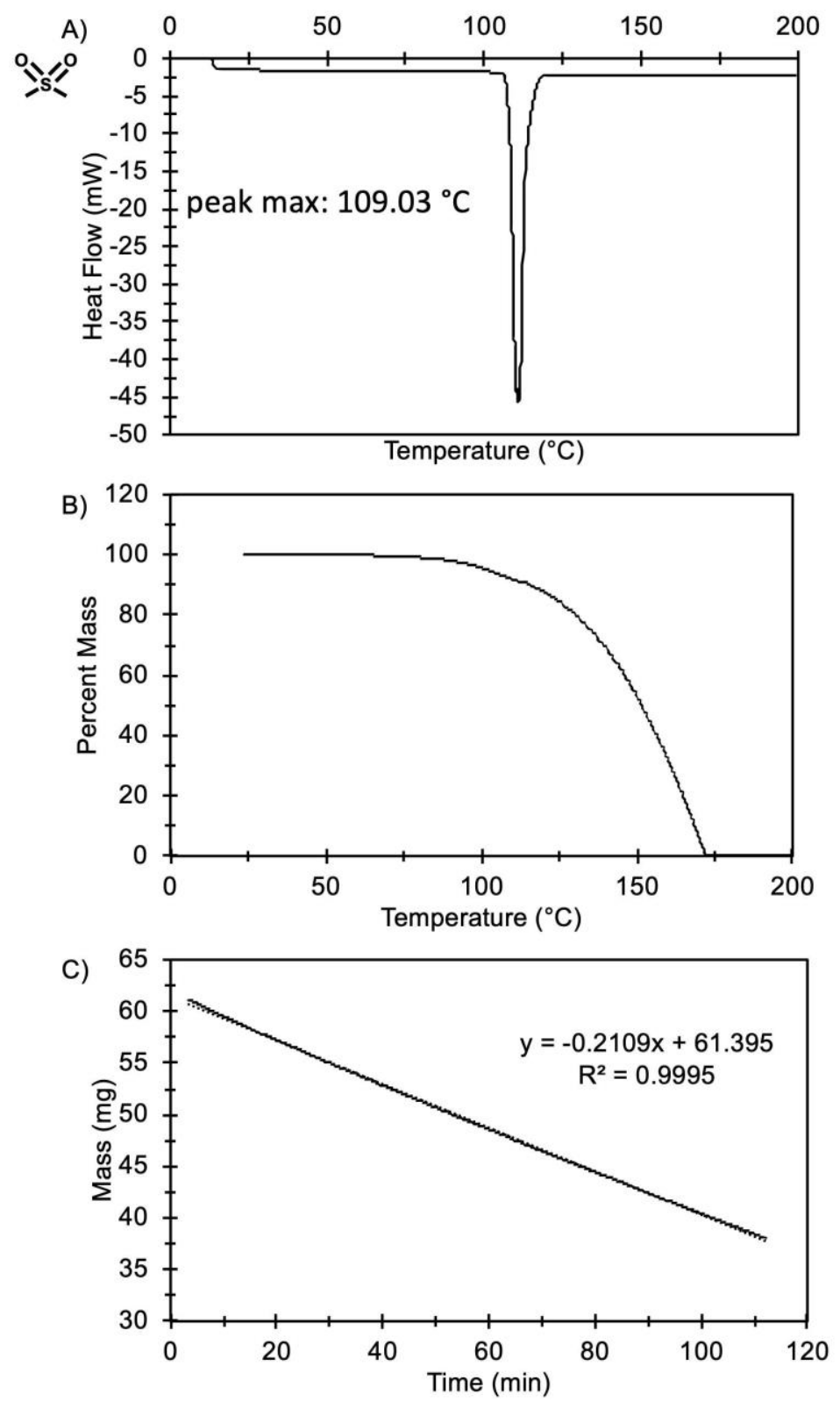

Figure S60. Thermal analysis of dimethyl sulfone. A) Differential scanning calorimetry.

B) Temperature ramped from room temperature to $350{ }^{\circ} \mathrm{C}$ at $10{ }^{\circ} \mathrm{C} \mathrm{min}^{-1}$. C) Temperature held constant at $91^{\circ} \mathrm{C}$. 

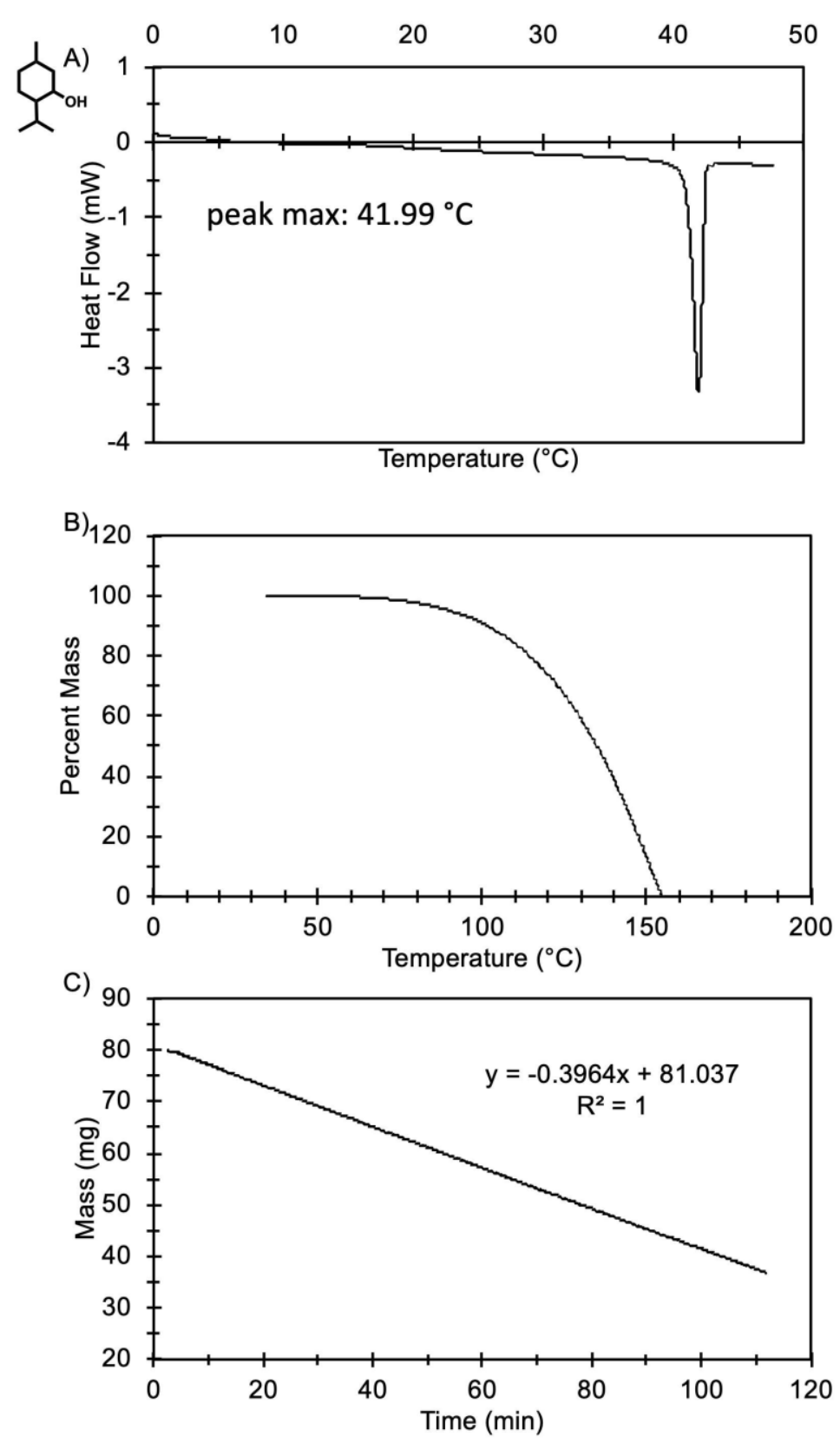

Figure S61. Thermal analysis of menthol. A) Differential scanning calorimetry. B) Temperature ramped from room temperature to $400^{\circ} \mathrm{C}$ at $10^{\circ} \mathrm{C} \mathrm{min}^{-1}$. C) Temperature held constant at $75^{\circ} \mathrm{C}$. 

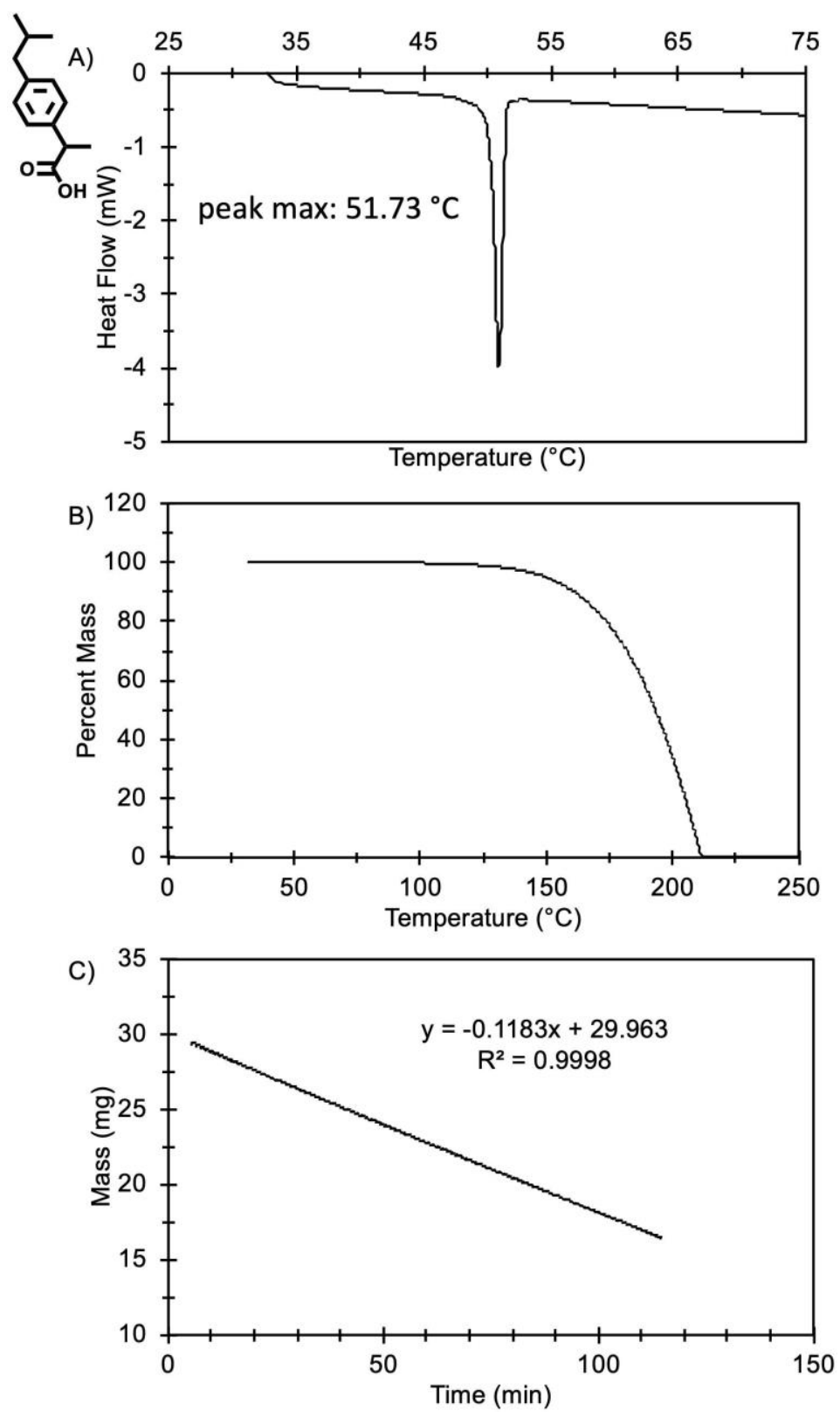

Figure S62. Thermal analysis of ibuprofen. A) Differential scanning calorimetry. B) Temperature ramped from room temperature to $350{ }^{\circ} \mathrm{C}$ at $10^{\circ} \mathrm{C} \mathrm{min}^{-1}$. C) Temperature held constant at $130{ }^{\circ} \mathrm{C}$. 


\section{SXIX. Modification of Substrate Surface Chemistry}

Glass slides were silanized using trichloro(octly)silane and trichloro $(1 \mathrm{H}, 1 \mathrm{H}, 1 \mathrm{H}, 2 \mathrm{H}, 2 \mathrm{H}$-perfluoro-octyl)silane by exposing the surface of the glass to the vapor of the silane under reduced pressure $(7 \mathrm{kPa})$ for $2 \mathrm{~h}$. Bonding of silanized glass slides with camphor or iodine, and the subsequent sample analyses were all performed as discussed above. Representative data for all analyses are illustrated in Figures S63-S66. 

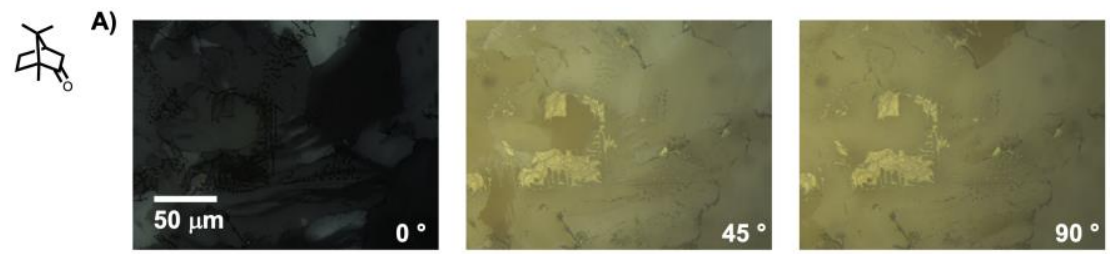

B)
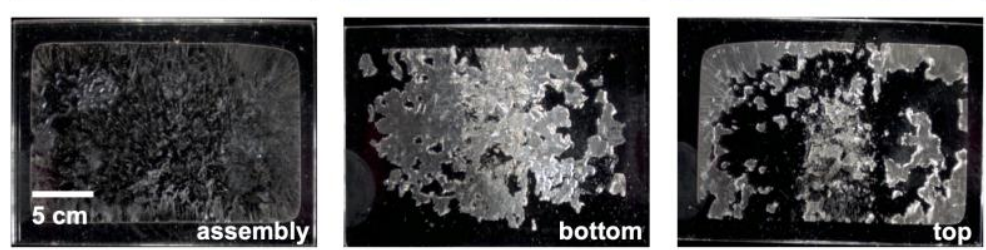

C)

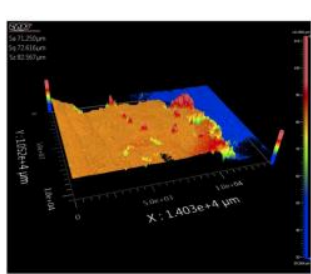

D)
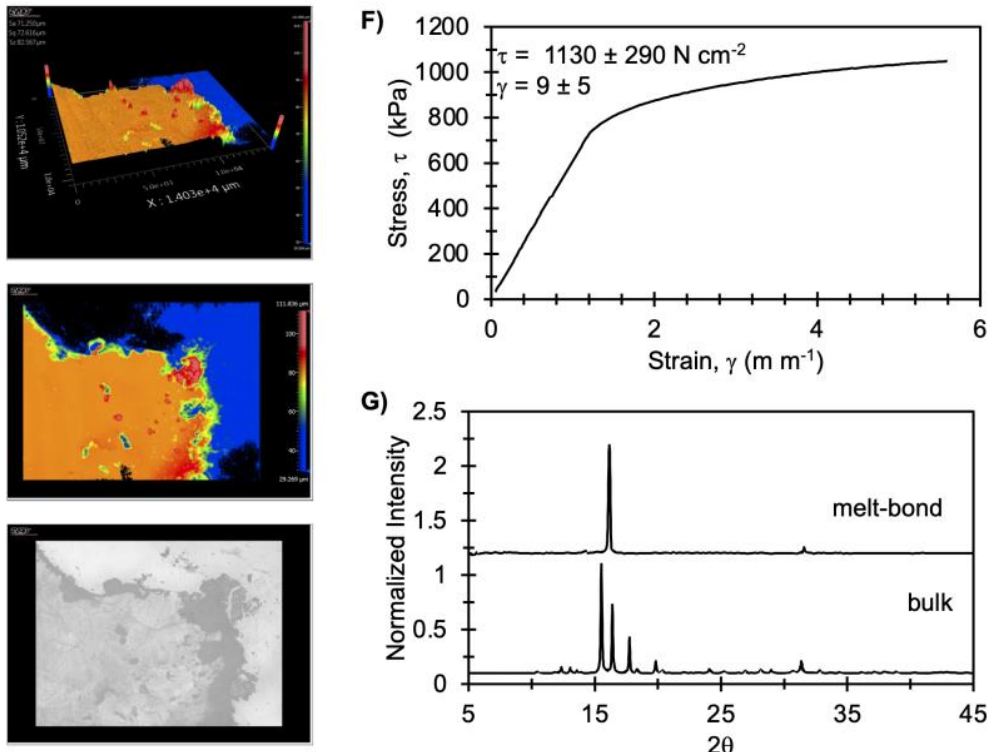

Figure S63. Representative data for glass silanized with trichloro $(1 \mathrm{H}, 1 \mathrm{H}, 1 \mathrm{H}, 2 \mathrm{H}, 2 \mathrm{H}-$ perfluoro-octyl)silane and bonded with camphor. A) Cross polarized light microscopy. B) Optical light microscopy. C) A 3-D profile of the sheared surface obtained from white light interferometry (WLI). D) A 2-D profile of the sheared surface obtained from WLI. E) A 2-D image of the sheared surface obtained from WLI. F) Representative stress-strain profile for. G) X-ray diffraction spectra of a melt-bonded and sheared sample compared to a bulk sample. 

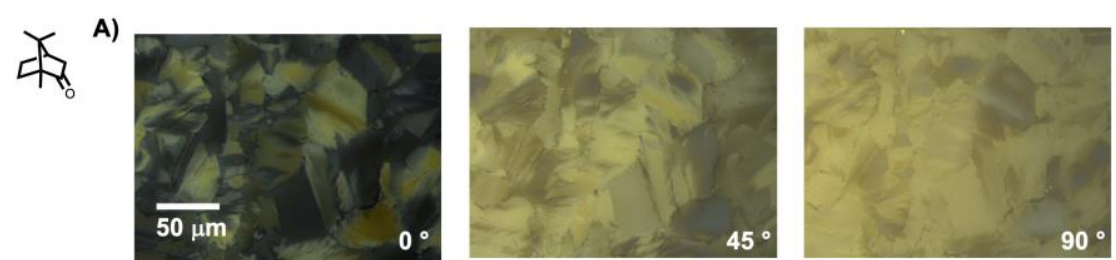

B)
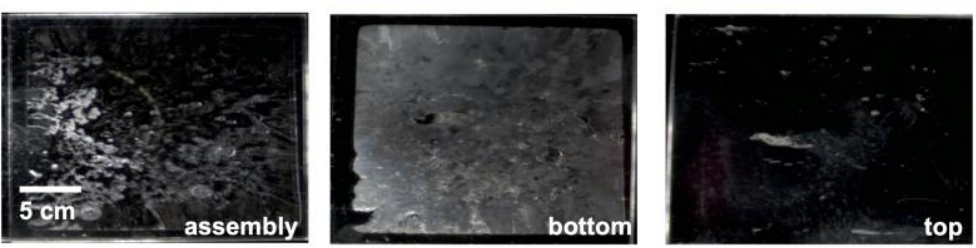

C)

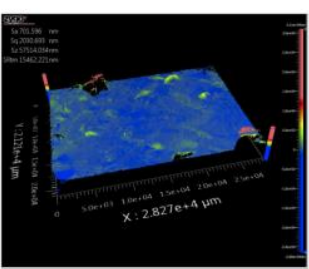

D)
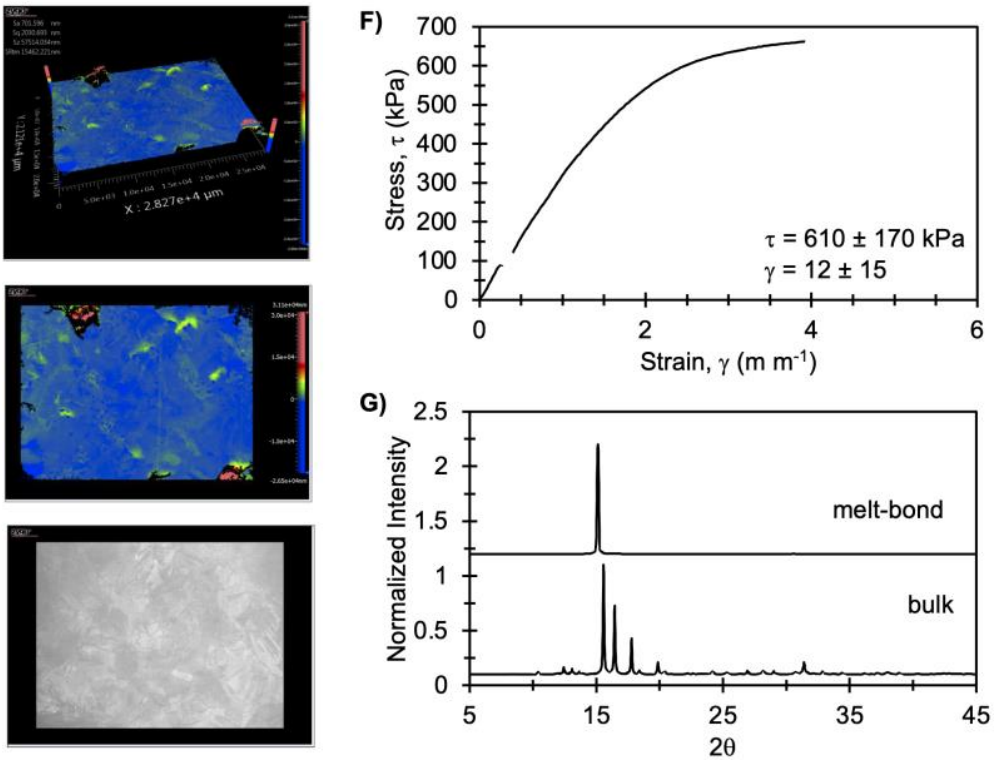

Figure S64. Representative data for glass silanized with trichloro(octyl)silane and bonded with camphor. A) Cross polarized light microscopy. B) Optical light microscopy. C) A 3-D profile of the sheared surface obtained from white light interferometry (WLI). D) A 2-D profile of the sheared surface obtained from WLI. E) A 2-D image of the sheared surface obtained from WLI. F) Representative stress-strain profile for. G) X-ray diffraction spectra of a melt-bonded and sheared sample compared to a bulk sample. 
$I_{2}$
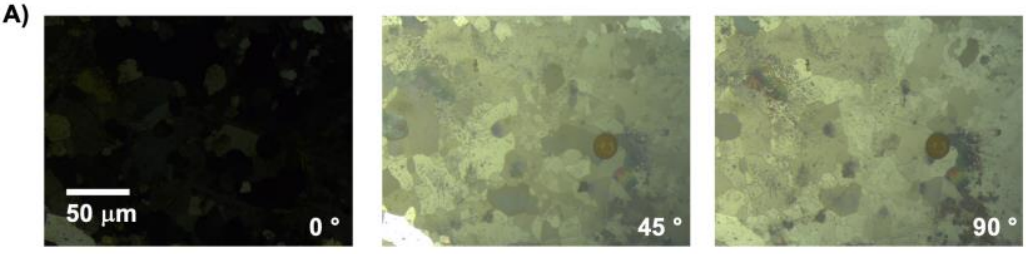

B)
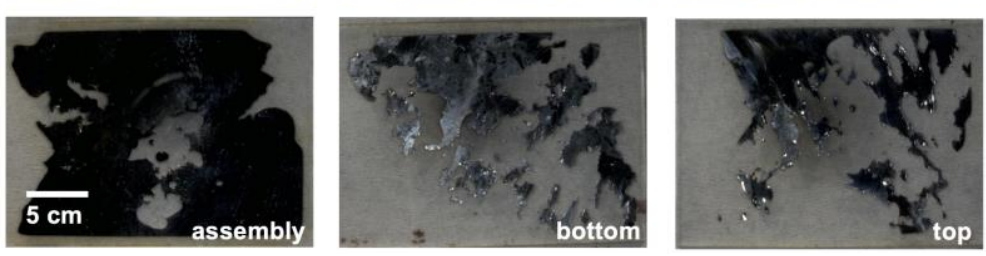

Figure S65. Representative data for glass silanized with trichloro $(1 \mathrm{H}, 1 \mathrm{H}, 1 \mathrm{H}, \mathbf{2 H}, \mathbf{2 H}-$ perfluoro-octyl)silane and bonded with iodine. A) Cross polarized light microscopy. B) Optical light microscopy. All samples failed prior to mechanical testing. 

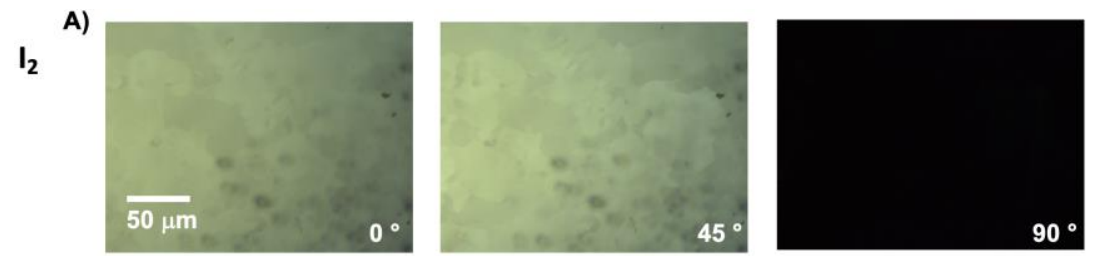

B)
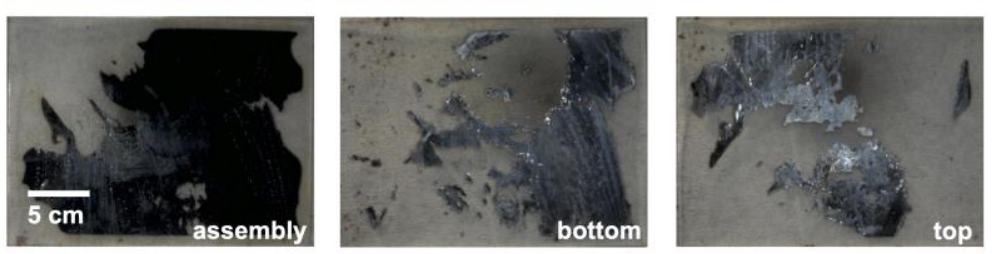

C)

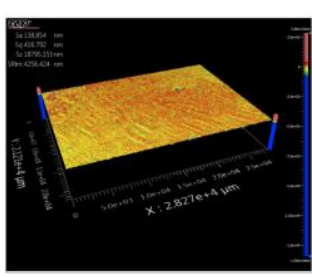

D)
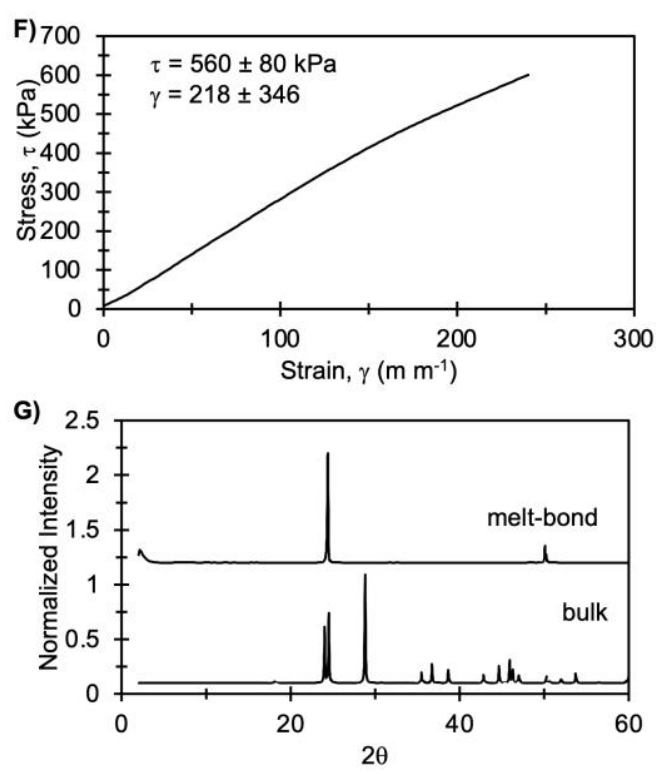

Figure S66. Representative data for glass silanized with trichloro(octyl)silane and bonded with iodine. A) Cross polarized light microscopy. B) Optical light microscopy. C) A 3-D profile of the sheared surface obtained from white light interferometry (WLI). D) A 2-D profile of the sheared surface obtained from WLI. E) A 2-D image of the sheared surface obtained from WLI. F) Representative stress-strain profile for. G) X-ray diffraction spectra of a meltbonded and sheared sample compared to a bulk sample. 


\section{SXX. Characterization of Glass Adherends}

Detailed procedures for modifying glass surfaces with silanes are provided in SI Section SXIX. Representative samples of all glass adherends were analyzed via contact angle goniometry and profilometry. The contact angle was measured using a contact angle goniometer (ramé-hart, Model 190-F2). A $2 \mu \mathrm{L}$ aliquot of deionized water was dispensed onto the surface of the substrate for each contact angle measurement. Values reported are the average of the left and right contact angles. Measured contract angles were 12,35 , and $74^{\circ}$ for unmodified glass, trichloro(octyl)silane-modified glass, and trichloro $(1 \mathrm{H}, 1 \mathrm{H}, 2 \mathrm{H}, 2 \mathrm{H}$-perfluorooctyl)silane-modified glass, respectively (Figure S67). 


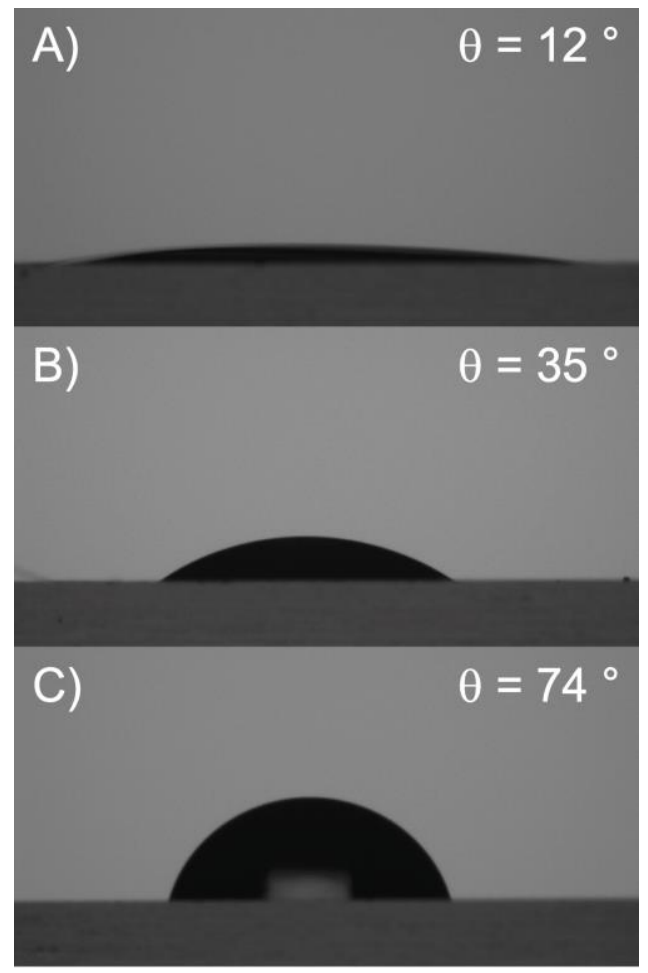

Figure S67. Contact angles $(\theta)$ of glass substrates with different surface chemistries.

A) Image from contact angle measurement for unmodified glass, a hydrophilic surface.

B) Image from contact angle measurement for trichloro(octyl)silane-modified glass.

C) Image from contact angle measurement for trichloro $(1 \mathrm{H}, 1 \mathrm{H}, 2 \mathrm{H}, 2 \mathrm{H}$ perfluorooctyl)silane-modified glass. 
The surface roughness average, $\mathrm{R}_{\mathrm{a}}$, for each material was measured using a profilometer (Tencor, Alpha Step 200). The $R_{a}$ is the average of the absolute height deviation value from the mean line determined by the profilometer. Surface roughness was measured over a $200-\mu \mathrm{m}$ distance with the diamond tip moving at a rate of $0.2 \mu \mathrm{m} \mathrm{s}^{-1}$. The measured surface roughness was $R_{a}=3 \pm 1 \mathrm{~nm}$ for all three substrates tested. These values within the range expected based on the previous literature. ${ }^{34}$ Furthermore, silanation of glass surfaces produces a conformal coating that is not expected to affect the surface roughness.

\section{SXXI. Optical Analysis of Bulk-Shear Samples}

Cross polarized light (XPL) micrographs were obtained using an XPL microscope (Nikon E200 POL) for two samples of each camphor and iodine. Each assembly was placed on a glass microscope slide on the rotatable stage of the XPL microscope, and micrographs were obtained with cross polarizers and stage rotations of $0^{\circ}, 45^{\circ}$, and $90^{\circ}$ in a region with a defining feature of interest.

The topology of the surface of the fractured cohesive later was characterized by white light interferometry (WLI) microscopy using a WLI microscope (Zygo NewView 5000 3D Surface Profiler). The WLI micrographs were obtained from three scans averaged with scan length of 145 $\mu \mathrm{m}$ in the preset "Micro" application setting of the Zygo Mx software. Representative XPL and WLI data are presented in Figures S68-S69. 

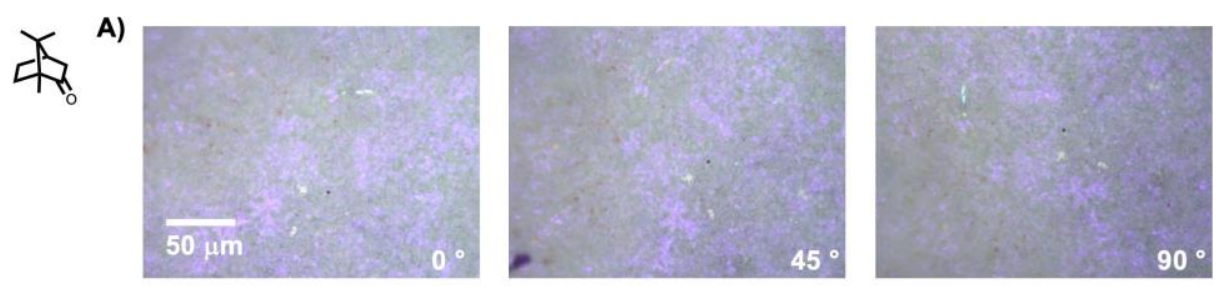

B)
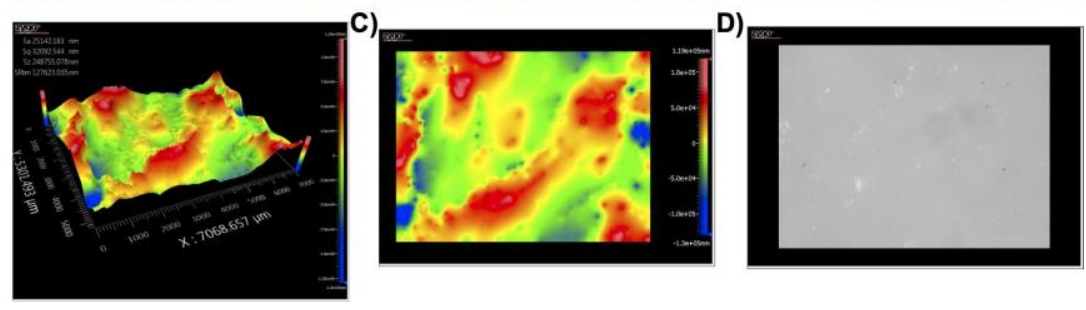

Figure S68. Representative data for bulk-shear samples of camphor. A) Cross polarized light microscopy. B) A 3-D profile of the sheared surface obtained from white light interferometry (WLI). C) A 2-D profile of the sheared surface obtained from WLI. D) A 2-D image of the sheared surface obtained from WLI. 


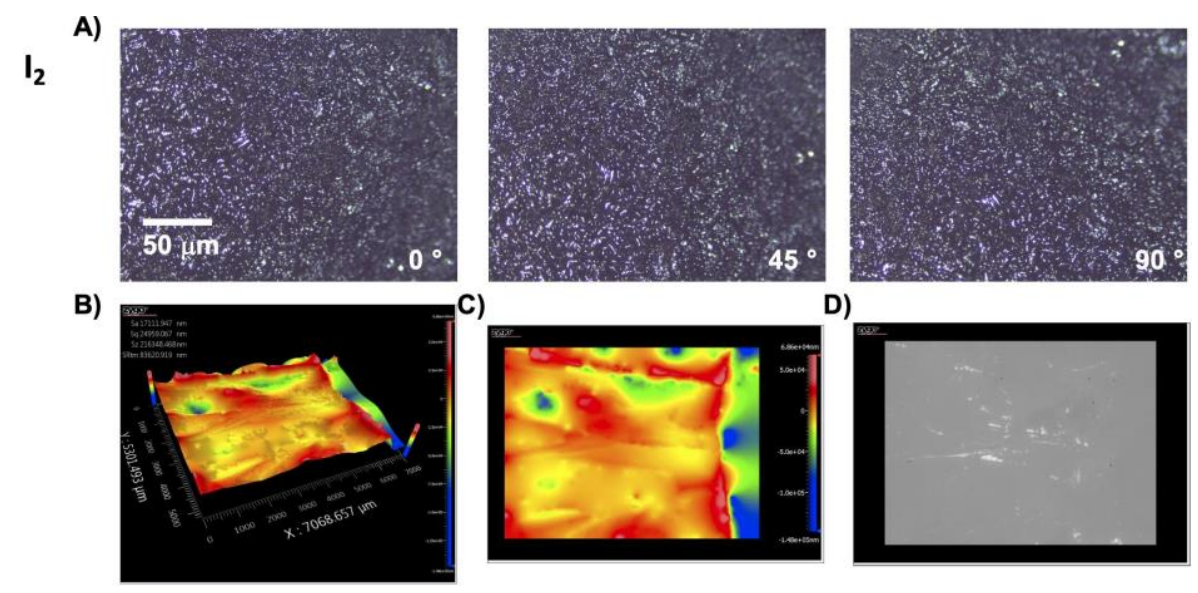

Figure S69. Representative data for bulk-shear samples of iodine. A) Cross polarized light microscopy. B) A 3-D profile of the sheared surface obtained from white light interferometry (WLI). C) A 2-D profile of the sheared surface obtained from WLI. D) A 2-D image of the sheared surface obtained from WLI. 


\section{SXXII.Single Crystal Growth and Structure Determination of (-)-Menthol}

A 19.7 M solution of (-)-menthol in cyclohexane was prepared by dissolving $1.5002 \mathrm{mg}$ of (-)-menthol in $0.5 \mathrm{~mL}$ solvent. The solution was placed in a 4-mL high-recovery vial (ChemGlass Life Sciences, Item \#: CG-4916-30), capped with an open-top cap and septa, and sonicated for 10 min to ensure complete dissolution of the solid. A hypodermic needle (20-gauge, Air-Tite Products Co., Inc., Cat. \#: N201) was inserted through the septa to allow slow evaporation of the solvent. Over time, complete evaporation of the solvent yielded colorless chunk-shaped single crystals of (-)-menthol.

Structure determination was completed by Dr. R. J. Staples at the Center for Crystallographic Research at Michigan State University. A suitable crystal $\left(0.32 \times 0.29 \times 0.21 \mathrm{~mm}^{3}\right)$ was selected and mounted on a nylon loop with paratone oil on a Bruker APEX-II CCD diffractometer. The crystal was kept at $\mathrm{T}=173 \pm 2 \mathrm{~K}$ during data collection. Using Olex $2{ }^{\mathrm{S}}{ }^{57}$ the structure was solved with the XT structure solution program, ${ }^{\mathrm{S} 36}$ using the Intrinsic Phasing solution method. The model was refined with version of XL using Least Squares minimization. ${ }^{\mathrm{S37}}$ Crystal data are presented in Table S6.

Table S6. Crystallographic data for (-)-menthol.

\begin{tabular}{cc}
\hline Formula & $\mathrm{C}_{10} \mathrm{H}_{20} \mathrm{O}$ \\
\hline$D_{\text {calc. }} / \mathrm{g} \mathrm{cm}^{-3}$ & 0.971 \\
$\mu / \mathrm{m} \mathrm{m}^{-1}$ & 0.060 \\
Formula Weight & 156.26 \\
Color & Colorless \\
Shape & Chunk \\
Size $/ \mathrm{mm}^{3}$ & $0.32 \times 0.29 \times 0.21$ \\
$T / \mathrm{K}$ & $173(2)$ \\
Crystal System & Trigonal \\
Flack Parameter & $0.1(9)$
\end{tabular}


Table S6. Crystallographic data for (-)-menthol (continued).

\begin{tabular}{|c|c|}
\hline Hooft Parameter & $-0.3(8)$ \\
\hline Space Group & $P 3_{1}$ \\
\hline$a / \AA$ & $21.452(4)$ \\
\hline$b / \AA$ & $21.452(4)$ \\
\hline$c / \AA$ & $6.0255(10)$ \\
\hline$\alpha /^{\circ}$ & 90 \\
\hline$\beta /^{\circ}$ & 90 \\
\hline$\gamma /{ }^{\circ}$ & 120 \\
\hline$V / \AA^{3}$ & $2405.3(9)$ \\
\hline$Z$ & 9 \\
\hline$Z^{\prime}$ & 3 \\
\hline Wavelength / Å & 0.710730 \\
\hline Radiation Type & $\operatorname{MoK}_{\alpha}$ \\
\hline$\Theta_{\min } /^{\circ}$ & 1.096 \\
\hline$\Theta_{\max } /^{\circ}$ & 26.098 \\
\hline Measured Reflections & 35094 \\
\hline Independent Reflections & 6365 \\
\hline Reflections Used & 4679 \\
\hline$R_{\text {int }}$ & 0.0922 \\
\hline Parameters & 319 \\
\hline Restraints & 1 \\
\hline Largest Peak & 0.194 \\
\hline Deepest Hole & -0.206 \\
\hline GooF & 1.042 \\
\hline$w R_{2}$ (all data) & 0.1621 \\
\hline$w R_{2}$ & 0.1435 \\
\hline$R_{1}$ (all data) & 0.0801 \\
\hline$R_{1}$ & 0.0577 \\
\hline
\end{tabular}

\section{SXXIII. Preparation and Characterization of Thermally Annealed Samples}

Lap-shear and bulk-shear samples of both camphor and octacyclic sulfur were prepared as described above. Pelleted samples were left in the PTFE mold and wrapped in aluminum foil during annealing. Once the samples were prepared, they were placed in an oven preheated to $90 \%$ the decomposition temperature by TGA, or $95 \%$ the absolute melting temperature, whichever was lower. Samples were removed from the oven after 1 hour, and processing/ 
characterization continued as described in the relevant SI sections. The average grain structure dimensions for representative samples were determined according to ASTM E112-13. ${ }^{38}$ Average resistance to stress and strain, and average grain size are reported in Table S7. Representative cross-polarized light micrographs, optical micrographs, white light interferometry profiles, and powder X-ray diffraction spectra are shown in Figures S71-SS73.

Table S7. Average resistance to stress and strain and average grain size for camphor and octacyclic sulfur samples.

\begin{tabular}{cccc}
\hline Sample & Mechanical Property & Non-Annealed & Annealed \\
\hline \multirow{2}{*}{ Camphor } & $\tau(\mathrm{kPa})$ & $1240 \pm 150$ & $980 \pm 290$ \\
Lap-Shear & $\gamma$ & $17 \pm 2$ & $6 \pm 4$ \\
& Grain Size & $1.6 \pm 0.6 \mathrm{~mm}$ & $96 \pm 23 \mu \mathrm{m}$ \\
Camphor & $\tau(\mathrm{kPa})$ & $740 \pm 250$ & $2100 \pm 560$ \\
Bulk-Shear & $\gamma$ & $0.70 \pm 0.53$ & $1.24 \pm 0.42$ \\
& Grain Size & $110 \pm 30 \mu \mathrm{m}$ & $109 \pm 37 \mu \mathrm{m}$ \\
Octacyclic & $\tau(\mathrm{kPa})$ & $360 \pm 60$ & 0 \\
Lap-Shear & $\gamma$ & $6 \pm 2$ & 0 \\
& Grain Size & $13 \pm 3 \mu \mathrm{m}$ & $91 \pm 45 \mu \mathrm{m}$ \\
Octacyclic & $\tau(\mathrm{kPa})$ & $1560 \pm 460$ & 0 \\
Bulk-Shear & $\gamma$ & $0.18 \pm 0.07$ & 0 \\
& Grain Size & $37 \pm 5 \mu \mathrm{m}$ & $81 \pm 21 \mu \mathrm{m}$ \\
\hline
\end{tabular}


A)

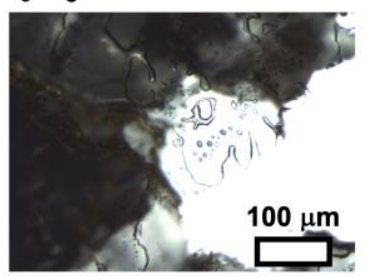

B)

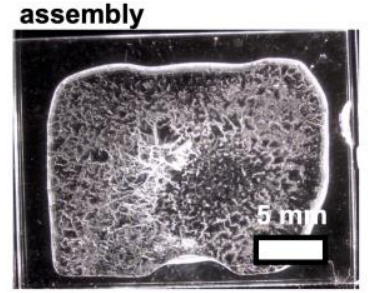

C) 3D

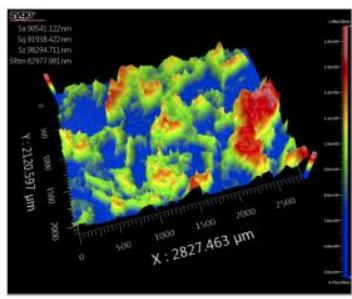

D)

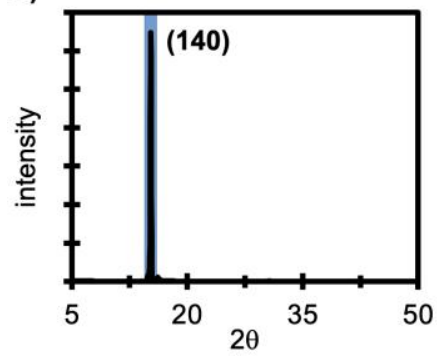

$\theta=45^{\circ}$

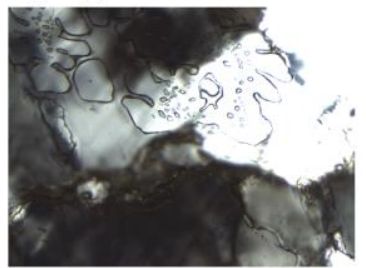

top

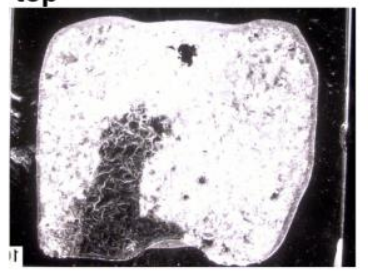

2D
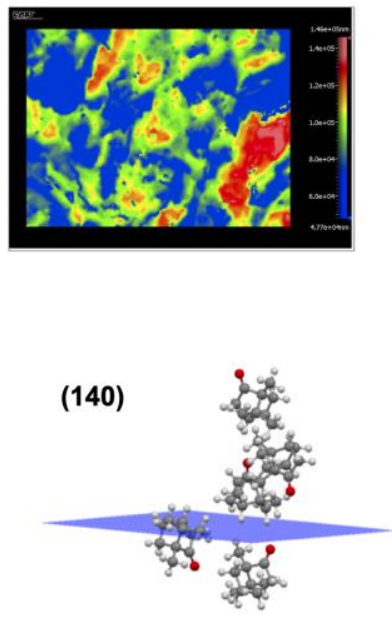

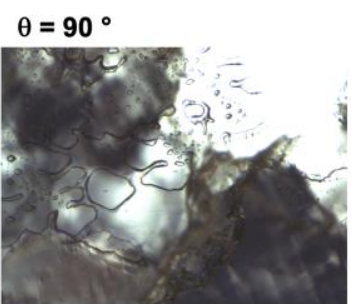

bottom

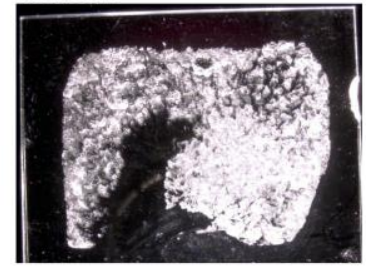

optical

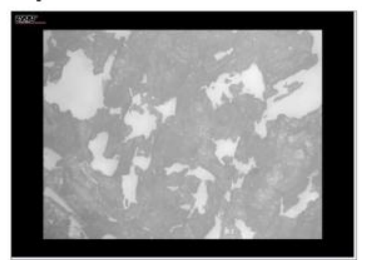

Figure S70. Representative data for a lap-shear sample of camphor thermally annealed at $48{ }^{\circ} \mathrm{C}$ for 1 hour. A) Cross-polarized light micrographs. B) Optical light micrographs. C) 3-D, 2-D, and optical white light interferometry profiles. D) Powder Xray diffraction spectra and preferred crystal orientation. 
A)

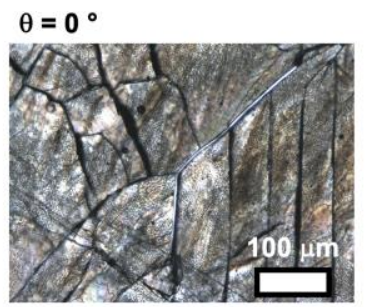

B)

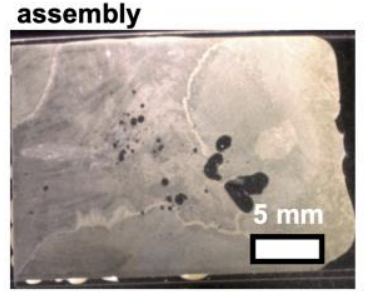

C) 3D

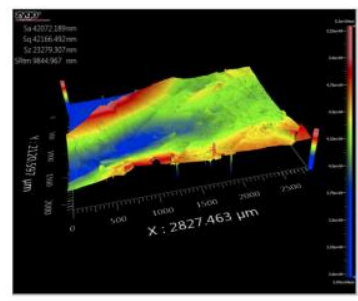

D)

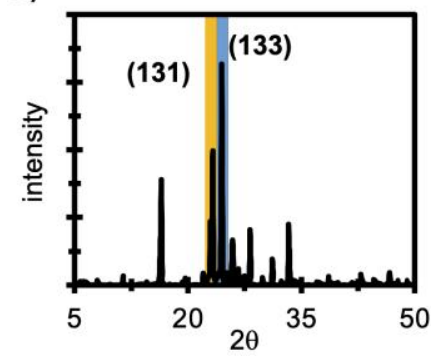

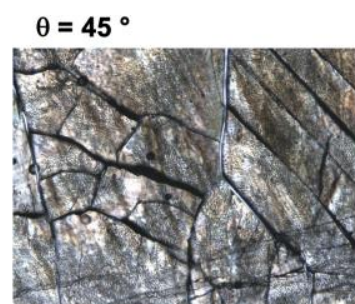

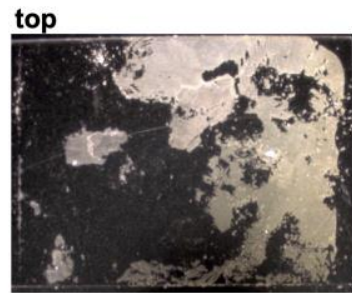

2D

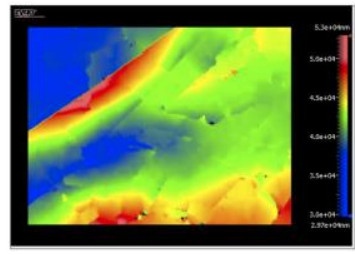

(133)

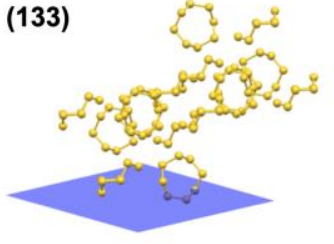

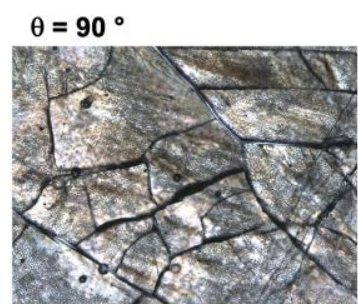

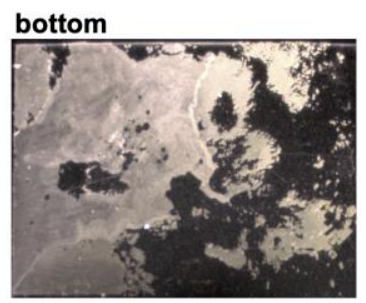

optical

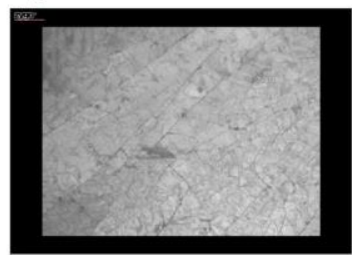

(131)

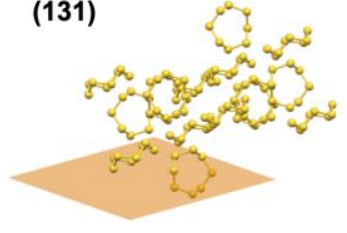

Figure S71. Representative data for a lap-shear sample of octacyclic sulfur thermally annealed at $95{ }^{\circ} \mathrm{C}$ for 1 hour. A) Cross-polarized light micrographs. B) Optical light micrographs. C) 3-D, 2-D, and optical white light interferometry profiles. D) Powder Xray diffraction spectra and preferred crystal orientation. 
A)

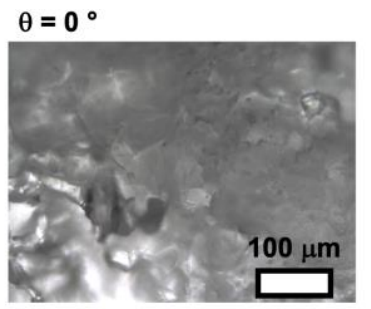

B) 3D

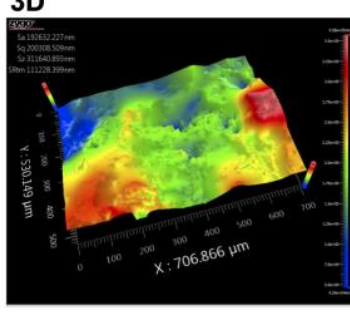

$\theta=45^{\circ}$

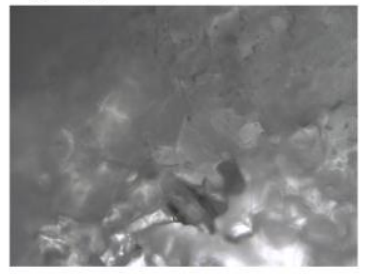

2D

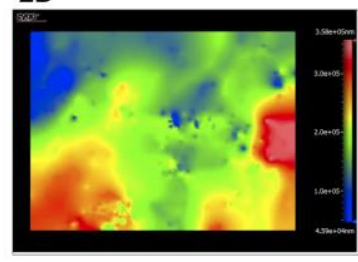

$\theta=90^{\circ}$

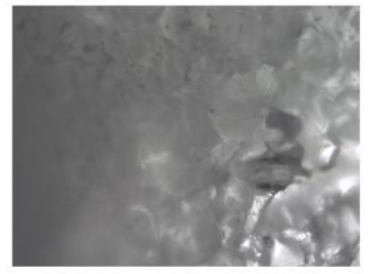

optical

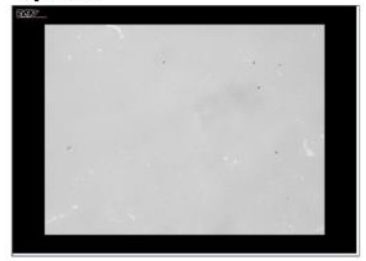

Figure S72. Representative data for a bulk-shear sample of camphor thermally annealed at $48^{\circ} \mathrm{C}$ for 1 hour. A) Cross-polarized light micrographs. B) 3-D, 2-D, and optical white light interferometry profiles. 
A)

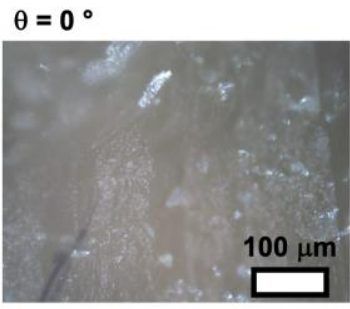

B) 3D

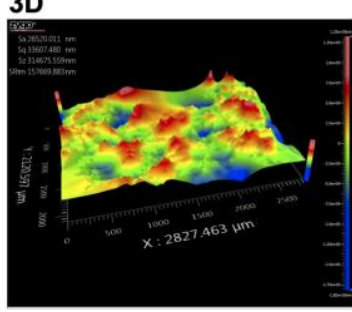

$\theta=45^{\circ}$

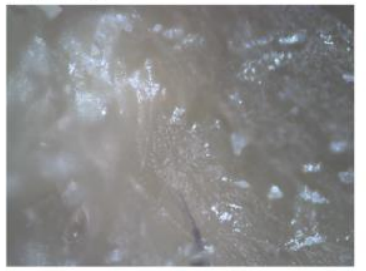

2D

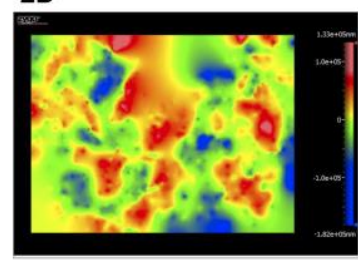

$\theta=90^{\circ}$

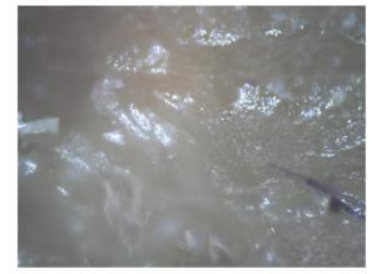

optical

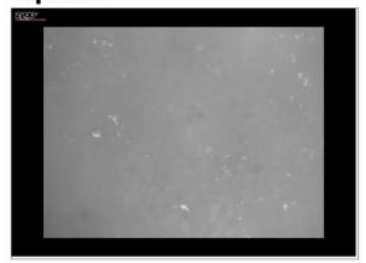

Figure S73. Representative data for a lap-shear sample of octacyclic sulfur thermally annealed at $95^{\circ} \mathrm{C}$ for 1 hour. A) Cross-polarized light micrographs. B) 3-D, 2-D, and optical white light interferometry profiles. 


\section{SXXIV. References}

(1) Peter J. Linstrom; W. Gary Mallard. NIST Chemistry WebBook. United States Department of Commerce 2018. https://doi.org/https://doi.org/10.18434/T4D303.

(2) Frederick, K. J.; Hildebrand, J. H. Specific Heats and Heat of Fusion of lodine. J. Am. Chem. Soc. 1938, 60 (6), 1436-1439. https://doi.org/10.1021/ja01273a048.

(3) Schwenz, R. W. Determination of the Heat of Sublimation. J. Chem. Educ. 1987, 64 (4), 551.

(4) Meyer, B. Elemental Sulfur. Chem. Rev. 1976, 76 (3), 367-388. https://doi.org/10.1021/cr60301a003.

(5) Sharma, B. L. L.; Gupta, S.; Tandon, S.; Kant, R. Physico-Mechanical Properties of Naphthalene-Acenaphthene Eutectic System by Different Modes of Solidification. Mater. Chem. Phys. 2008, 111 (2-3), 423-430.

https://doi.org/10.1016/J.MATCHEMPHYS.2008.04.049.

(6) Torres-Gómez, L. A.; Barreiro-Rodríguez, G.; Galarza-Mondragón, A. A New Method for the Measurement of Enthalpies of Sublimation Using Differential Scanning Calorimetry. Thermochim. Acta 1988, 124, 229-233. https://doi.org/10.1016/0040-6031(88)87025-4.

(7) Solomonov, B. N.; Varfolomeev, M. A.; Nagrimanov, R. N.; Novikov, V. B.; Ziganshin, M. A.; Gerasimov, A. V.; Verevkin, S. P. Enthalpies of Vaporization and Sublimation of the Halogen-Substituted Aromatic Hydrocarbons at 298.15 K: Application of Solution Calorimetry Approach. J. Chem. Eng. Data 2015, 60 (3), 748-761. https://doi.org/10.1021/je5008795.

(8) Verevkin, S. P.; Emel'yanenko, V. N.; Klamt, A. Thermochemistry of Chlorobenzenes and 
Chlorophenols: Ambient Temperature Vapor Pressures and Enthalpies of Phase

Transitions. Jounral Chem. Eng. Data 2007, 52, 499-510.

https://doi.org/10.1021/je060429r.

(9) Frandsen, M. Cryscopic Constant, Heat of Fusion, and Heat Capacity of Camphor. Bur.

Stand. J. Res. 1931, No. 7, 477-483.

(10) Chickos, J. S. A Simple Equilibrium Method for Determining Heats of Sublimation. J.

Chem. Educ. 1975, 52 (2), 134-136. https://doi.org/10.1021/ed052p134.

(11) Domalski, E. S.; Hearing, E. D. Heat Capacities and Entropies of Organic Compounds in the Condensed Phase. Volume III. J. Phys. Chem. Ref. Data 1996, 25 (1), 1-525.

https://doi.org/10.1063/1.555985.

(12) Busfield, W. K.; Mackle, H.; O'Hare, P. A. G. Studies in the Thermochemistry of Sulphones. Part 2.-The Standard Heats of Formation of Sulphones of the Type RSO2CH3. Trans. Faraday Soc. 1961, 57, 1054-1057. https://doi.org/10.1039/TF9615701054.

(13) Chickos, J. S.; Garin, D. L.; Hitt, M.; Schilling, G. Some Solid State Properties of Enantiomers and Their Racemates. Tetrahedron 1981, 37 (12), 2255-2259. https://doi.org/10.1016/S0040-4020(01)97981-5.

(14) Hong, J.; Hua, D.; Wang, X.; Wang, H.; Li, J. Solid-Liquid-Gas Equilibrium of the Ternaries Ibuprofen + Myristic Acid + CO2 and Ibuprofen + Tripalmitin + CO2. J. Chem. Eng. Data 2010, 55 (1), 297-302. https://doi.org/10.1021/je900342a.

(15) Maxwell, R.; Chickos, J. An Examination of the Thermodynamics of Fusion, Vaporization, and Sublimation of Ibuprofen and Naproxen by Correlation Gas Chromatography. J. Pharm. Sci. 2012, 101 (2), 805-814. https://doi.org/10.1002/JPS.22803. 
(16) McQuarrie, D. A.; Simon, J. D. Physical Chemistry: A Molecular Approach, 1st ed.; University Science Books: Herndon, VA, 1997.

(17) Harris, P. M.; Mack, E.; Blake, F. C. The Atomic Arrangement in the Crystal of Orthorhomic lodine. J. Am. Chem. Soc. 1928, 50 (6), 1583-1600.

https://doi.org/10.1021/ja01393a009.

(18) van Bolhuis, F.; Koster, P. B.; Migchelsen, T. Refinement of the Crystal Structure of lodine at $110^{\circ}$ K. Acta Crystallogr. 1967, 23 (1), 90-91.

https://doi.org/10.1107/S0365110X6700218X.

(19) Oddershede, J.; Larsen, S. Charge Density Study of Naphthalene Based on X-Ray Diffraction Data at Four Different Temperatures and Theoretical Calculations. J. Phys. Chem. A 2004, 108 (6), 1057-1063. https://doi.org/10.1021/JP036186G.

(20) Reddy, C. M.; Kirchner, M. T.; Gundakaram, R. C.; Padmanabhan, K. A.; Desiraju, G. R. Isostructurality, Polymorphism and Mechanical Properties of Some Hexahalogenated Benzenes: The Nature of Halogen $\cdots$ Halogen Interactions. Chem. - A Eur. J. 2006, 12 (8), 2222-2234. https://doi.org/10.1002/chem.200500983.

(21) Brunelli, M.; Fitchci, A. N.; Mora, A. J. Low-Temperature Crystal Structure of S-Camphor Solved from Powder Synchrotron X-Ray Diffraction Data by Simulated Annealing. J. Solid State Chem. 2002, 163 (1), 253-258.

https://doi.org/https://doi.org/10.1006/jssc.2001.9400.

(22) Fronczek, F. R. CCDC 638426: Experimental Crystal Structure Determination. CSD Commun. 2007. https://doi.org/10.5517/ccpfbdt.

(23) Ostrowska, K.; Kropidłowska, M.; Katrusiak, A. High-Pressure Crystallization and 
Structural Transformations in Compressed R,S-Ibuprofen. Cryst. Growth Des. 2015, 15 (3), 1512-1517. https://doi.org/10.1021/cg5018888.

(24) Baxter, G. P.; Hickey, C. H.; Holmes, W. C. The Vapor Pressure of lodine. J. Am. Chem. Soc. 1907, 29 (2), 127-136. https://doi.org/10.1021/ja01956a004.

(25) McEachern, D. M. A Molecular Flow Evaporation Apparatus for Measuring Vapour Pressures and Heats of Sublimation of Organic Compounds. J. Phys. E. 1973, 6, 155-161.

(26) Jones, A. H. Sublimation Pressure Data for Organic Compounds. J. Chem. Eng. Data 1960, 5 (2), 196-200. https://doi.org/10.1021/je60006a019.

(27) Stull, D. R. Vapor Pressure of Pure Substances. Organic and Inorganic Compounds. Ind. Eng. Chem. 1947, 39 (4), 517-540. https://doi.org/10.1021/ie50448a022.

(28) Daubert, T. E.; Danner, R. P. Physical and Thermodynamic Properties of Pure Chemicals: Data Compilation; Taylor \& Francis: Washington, D.C., 1989.

(29) Standard Test Method for Apparent Shear Strength of Single-Lap-Joint Adhesively Bonded Metal Specimens by Tension Loading (Metal-to-Metal); West Conshohocken, PA, 2018. https://doi.org/10.1520/D1002-10.

(30) Pecharsky, V.; Zavalij, P. Fundamentals of Powder Diffraction and Structural Characterization of Materials, 2nd ed.; Springer International Publishing: New York City, NY, 2009.

(31) Spackman, M. A.; Jayatilaka, D. Hirshfeld Surface Analysis. Cryst. Eng, Comm. 2009, 11 (1), 19-32. https://doi.org/10.1039/b818330a.

(32) Spackman, M. A.; McKinnon, J. J. Fingerprinting Intermolecular Interactions in Molecular Crystals. CrystEngComm 2002, 4 (66), 378-392. https://doi.org/10.1039/B203191B. 
(33) Standard Test Method for Shear Strength of Plastics by Punch Tool; West Conshohocken, PA, 2017.

(34) Mitchell, H. T.; Smith, M. K.; Blelloch, N. D.; Van Citters, D. W.; Mirica, K. A. Polycyclic Aromatic Hydrocarbons as Sublimable Adhesives. Chem. Mater. 2017, 29 (7), 2788-2793. https://doi.org/10.1021/acs.chemmater.6b04641.

(35) Dolomanov, O. V.; Bourhis, L. J.; Gildea, R. J.; Howarda, J. A. K.; Puschmanna, H. OLEX2: A Complete Structure Solution, Refinement and Analysis Program. J. Appl. Crystallogr. 2009, 42 (2), 339-341. https://doi.org/10.1107/S0021889808042726.

(36) Sheldrick, G. M. A Short History of $\{\backslash$ it SHELX $\}$. Acta Crystallogr. Sect. A 2008, 64 (1), 112122. https://doi.org/10.1107/S0108767307043930.

(37) Sheldrick, G. M. Crystal Structure Refinement with $\{\backslash$ it SHELXL\}. Acta Crystallogr. Sect. C 2015, 71 (1), 3-8. https://doi.org/10.1107/S2053229614024218.

(38) E112-13 Standard Test Methods for Determineing Average Grain Size; West Conshohocken, PA, United States, 2013. https://doi.org/10.1520/E0112-13. 